Portland State University

PDXScholar

Summer 8-5-2014

\title{
Optical Properties of Nanostructured Dielectric Coatings
}

Brandon Giatti

Portland State University

Follow this and additional works at: https://pdxscholar.library.pdx.edu/open_access_etds

Part of the Nanoscience and Nanotechnology Commons Let us know how access to this document benefits you.

Recommended Citation

Giatti, Brandon, "Optical Properties of Nanostructured Dielectric Coatings" (2014). Dissertations and Theses. Paper 1940.

https://doi.org/10.15760/etd.1939

This Thesis is brought to you for free and open access. It has been accepted for inclusion in Dissertations and Theses by an authorized administrator of PDXScholar. Please contact us if we can make this document more accessible: pdxscholar@pdx.edu. 
Optical Properties of Nanostructured Dielectric Coatings

by

Brandon Giatti

A thesis submitted in partial fulfillment of the requirements for the degree of

Master of Science

in

Physics

Thesis Committee:

Rolf Koenenkamp, Chair

Erik Bodegom

Ralf Widenhorn

Portland State University

2014 


\begin{abstract}
Solar cells have extrinsic losses from a variety of sources which can be minimized by optimization of the design and fabrication processes. Reflection from the front surface is one such loss mechanism and has been managed in the past with the usage of planar antireflection coatings. While effective, these coatings are each limited to a single wavelength of light and do not account for varying incident angles of the incoming light source. Three-dimensional nanostructures have shown the ability to inhibit reflection for differing wavelengths and angles of incidence. Nanocones were modeled and show a broadband, multi-angled reflectance decrease due to an effective grading of the index.
\end{abstract}

Finite element models were created to simulate incident light on a zinc oxide nanocone textured silicon substrate. Zinc oxide is advantageous for its ease of production, benign nature, and refractive index matching to the air source region and silicon substrate. Reflectance plots were computed as functions of incident angle and wavelength of light and compared with planar and quintic refractive index profile models. The quintic profile model exhibits nearly optimum reflection minimization and is thus used as a benchmark. Physical quantities, including height, width, density, and orientation were varied in order to minimize the reflectance. A quasi-random nanocone unit cell was modeled to better mimic laboratory results. The model was comprised of 10 nanocones with differing structure and simulated a larger substrate by usage of periodic boundary conditions. The simulated reflectance shows a $\sim 50 \%$ decrease when compared with a planar model. When a seed layer is added, simulating a layer of non-textured zinc oxide, on which the nanocones are grown, the reflectance shows a fourfold decrease when compared with 
planar models. At angles of incidence higher than $75^{\circ}$, the nanocone model outperformed the quintic model. 


\section{ACKNOWLEDGEMENTS}

\section{I would like to thank the following individuals:}

- Dr. Rolf Koenenkamp for his guidance and patience

- Dr. Erik Bodegom and Dr. Ralf Widenhorn for their participation in sitting on my thesis committee

- Dr. Robert Word for his help in navigating the difficulties of this work

- Dr. Peter Leung, Dr. John Freeouf, and Dr. Erik Sanchez for their guidance

- Joseph Fitzgerald and Mathieu Valat for help with assembling the hardware and enabling the software needed to complete the models

- My family, friends, and those who inspired me

- My loving wife Christine, without whom I could not accomplish this task 


\section{TABLE OF CONTENTS}

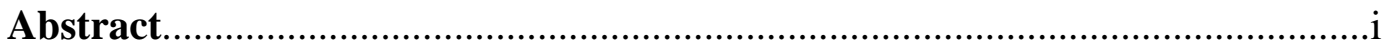

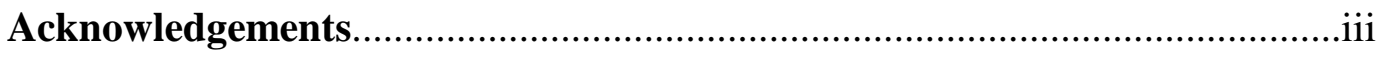

List of Tables......................................................................................

List of Figures....................................................................................

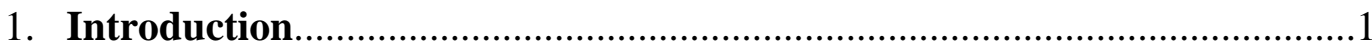

1.1. Solar Cell Operation and Structure.......................................................2

1.2. Efficiency Limitations and Design Improvements.................................4

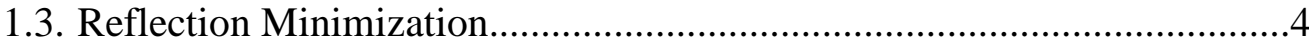

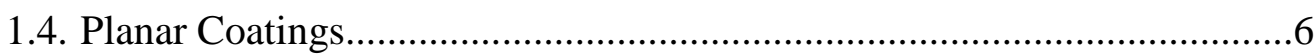

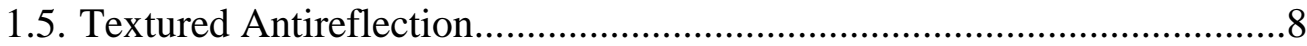

1.6. Zinc Oxide Nanocones as Antireflection Materials..................................9

1.7. Motivation for Computation and Effective Medium Approximation.......10

1.8. Thesis and Research Objectives.......................................................12

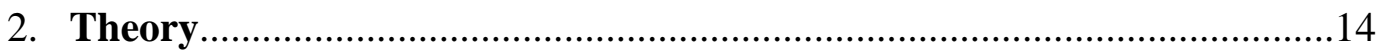

2.1. Maxwell's Electrodynamic Equations................................................14

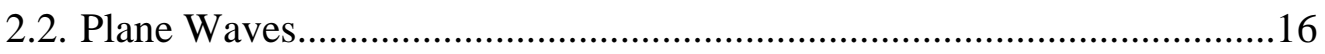

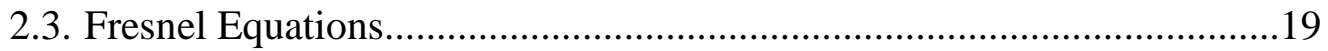

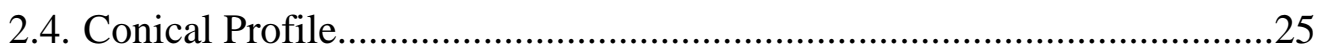

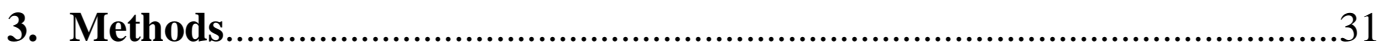

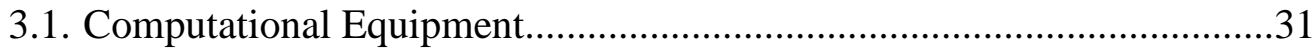

3.2. Finite Element Method and PARDISO Solver........................................32

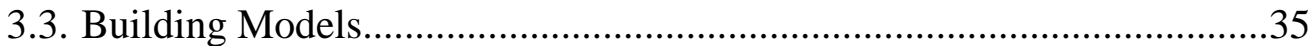




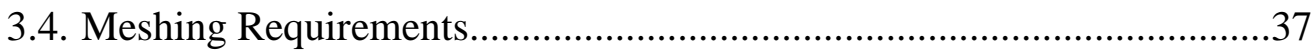

3.5. Nanocone Model Geometry and Mesh..................................................37

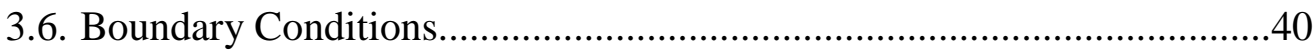

3.6.1. Continuity Boundary Conditions....................................................41

3.6.2. Periodic Boundary Conditions..........................................................43

3.6.3. Port Boundary Conditions..............................................................

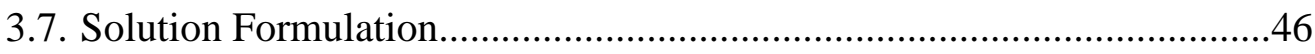

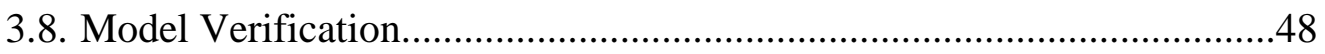

3.8.1. Periodic Boundary Conditions from Single Cell Grouping............49

3.8.2. Fresnel Model Verification...........................................................51

3.8.3. Discounting Absorption...................................................................53

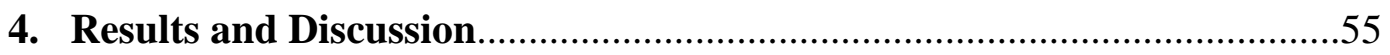

4.1. Nanocone Verification Model.................................................................55

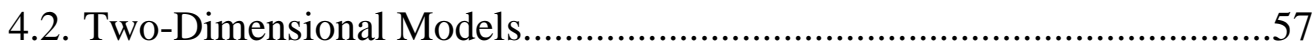

4.3. Three-Dimensional Models......................................................................

4.3.1. Single Nanocone with Periodic Boundaries.....................................59

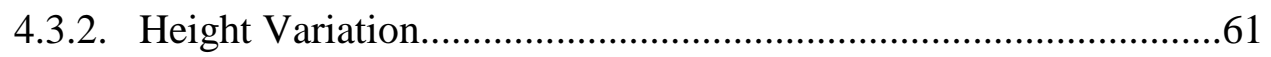

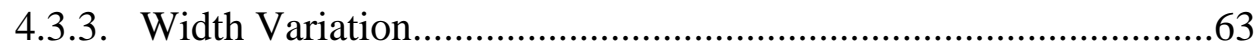

4.3.4. Density Variation..........................................................................65

4.4. Nanocone Comparison with Quintic Profile Gradient Index Films.........68

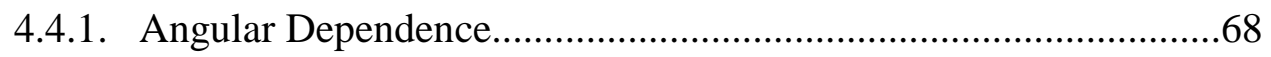

4.4.2. Wavelength Dependence...............................................................70

4.5. Quasi-Randomized Nanocone Model with Periodic Unit Cells................71 
4.6. Seed Layer.

4.7. Comparison with Laboratory Results..

4.8. Wavelength Dependence of Quasi-Random

Nanocone Model with Seed Layer.....................................................992

4.9. Discussion and Future Work...........................................................93

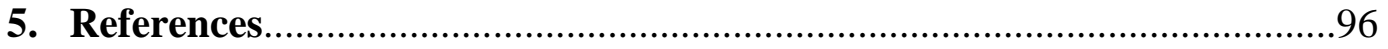




\section{LIST OF TABLES}

Table 3.1. Computer hardware required for model simulations...........................31

Table 4.1. Randomization parameters for 3D quasi-random nanocone model......72

Table 4.2. Type and quantity of 3D quasi-random

nanocone model mesh elements......................................................73 


\section{LIST OF FIGURES}

Figure 1.1. Electron microscope image of moth eye........................................2

Figure 1.2. Reflection of incident light on a solar cell.......................................5

Figure 1.3. Single planar antireflective coating............................................6

Figure 1.4. Light trapping on textured surface.............................................. 9

Figure 1.5. Laboratory constructed nanocones...........................................10

Figure 1.6. Lab grown zinc oxide nanowires................................................... 12

Figure 2.1. Incident, reflected, and transmitted fields of a TE wave...................18

Figure 2.2. S-polarized wave reflecting and transmitting.................................21

Figure 2.3. P-polarized wave reflecting and transmitting.................................23

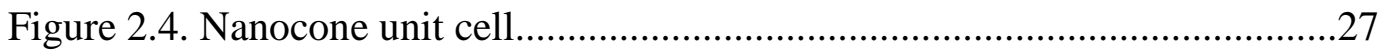

Figure 2.5. Nanocone and quintic refractive index profiles..............................29

Figure 2.6. Nanocone with seed layer refractive index profile............................30

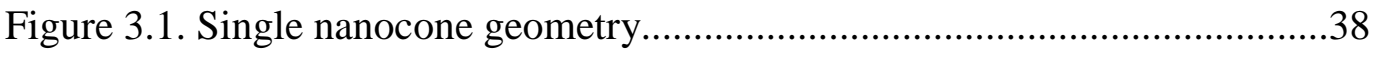

Figure 3.2. Nanocone mesh....................................................................40

Figure 3.3. Two-dimensional model boundary conditions...............................41

Figure 3.4. Continuity of electric fields on an arbitrary surface.........................42 viii 
Figure 3.5. Normalized E-field incident on 2D nanopillars

Figure 3.6. Comparison of 2D models with 5 domains and periodicity.

Figure 3.7. Calculated and simulated results of Fresnel model. .52

Figure 3.8. Comparison of real and complex dielectric

functions for Air/Si interface. .54

Figure 4.1. Height reduction of nanocones..................................................56

Figure 4.2. Reflectance plots for decreasing nanocone heights. .57

Figure 4.3. Nanoridge field plots for TM and TE propagation. .58

Figure 4.4. Reflectance vs. angle plots for 2D nanoridges .58

Figure 4.5. Single nanocone unit cell .59

Figure 4.6. Nanocone and planar reflectance vs. angle plots .60

Figure 4.7. Varied nanocone heights reflectance vs. angle plots for TM. .61

Figure 4.8. Varied nanocone heights reflectance vs. angle plots for TE. .62

Figure 4.9. Comparison of nanocone radii .63

Figure 4.10. Varied nanocone widths reflectance vs. angle plots for TM. .64

Figure 4.11. Varied nanocone widths reflectance vs. angle plots for TE. .64

Figure 4.12. Model domains for varied density........ .66 
Figure 4.13. Varied nanocone density reflectance vs. angle plots for TM.

Figure 4.14. Varied nanocone density reflectance vs. angle plots for TE.

Figure 4.15. Nanocone and quintic refractive index profiles .68

Figure 4.16. Reflectance vs. angle plots for quintic, planar, and nanocones .69

Figure 4.17. TM reflectance vs. wavelength plots for quintic, planar, and nanocones. .70

Figure 4.18. TE reflectance vs. wavelength plots for quintic, planar, and nanocones. .71

Figure 4.19. Top view of quasi-randomized nanocone model. .73

Figure 4.20. Top view of quasi-randomized nanocone model mesh .74

Figure 4.21. Quasi-randomized nanocone model mesh at 45 degree angle .75

Figure 4.22. Field solutions for TM and TE quasi-randomized nanocone model .76

Figure 4.23. Reflectance vs. angle plots for quasi-randomized nanocones and planar. .77

Figure 4.24. Nanocone and planar reflectance vs. angle plots for comparison.....78

Figure 4.25. Quasi-random nanocone reflectance plots with coarse mesh. .80 
Figure 4.26. Reflectance plot for outlier check around 39 degrees.

Figure 4.27. Refractive index profiles for quintic and seed layer model. .82

Figure 4.28. Fields for seed layer model .83

Figure 4.29. Reflectance comparison of nanocone and seed layer models .84

Figure 4.30. Quasi-random nanocones with seed layer mesh .85

Figure 4.31. Reflectance vs. angle for quasi-random model with seed layer........86

Figure 4.32. Reflectance plot for outlier check around 48 degrees .87

Figure 4.33. Reflectance plot for outlier check around 72 degrees.....................88

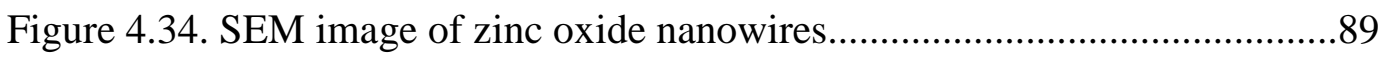

Figure 4.35. Quasi-random nanocone computational model.............................90

Figure 4.36. SEM image of zinc oxide nanowires for density calculation. . .91

Figure 4.37. Experimental reflectance from randomly oriented $\mathrm{ZnO}$ nanowires .92

Figure 4.38. Reflectance vs. wavelength for quasi-random nanocone geometry .93 


\section{Introduction}

Reflection occurs when light travels from one medium to another with differing refractive indices. The minimization of reflection is of great importance to a number of scientific endeavors and technological fields, including solar power. Photovoltaic energy production is a continually growing field which has experienced rapid growth throughout the past decade. Installed solar capacity more than doubled from 2010 to 2011, up from 887 megawatts to 1,887 megawatts of total capacity in the United States [1]. The upward trend continued in 2012 as capacity reached 3,313 megawatts [2]. Despite a recent natural gas boom, solar technology is positioned for continued growth in the coming decades. While the technology is not novel, and silicon solar cells have been in use for more than half-a-century, the power produced is still marginal when compared to fossil fuel power production due primarily to photovoltaic cells being much less cost-efficient. Reducing the production costs will allow solar technology to thrive as an alternative to the more pollution-intensive, geopolitically-risky, climate-altering fossil fuel power industry. Perhaps the most advantageous attribute of solar energy is the abundance of sunlight the Earth receives. In terms of fossil fuel usage, tons of coal equivalence is used, the solar radiation per year is equal to $1.8 \times 10^{18}$ tons of coal equivalence [3]. With such a great amount of solar irradiance, even utilization of a modest percentage would allow for much of the world's power needs to be met.

The need for better performing solar cells has led researchers to study the characteristics of animal antireflective (AR) structures. The usage of nature's designs in science and engineering is known as biomimetics. Some insects use AR to camouflage themselves 
from predators, while others use AR to enhance vision [4]. The eyes of moths have developed over millions of years to be antireflective due to nano-pillars which are closely-packed on the surface of the eye [5]. The usage of structures to minimize reflection, as used in nature, has many applications including military camouflage, optical lenses, and solar cells. The solar cell application is of particular interest here.

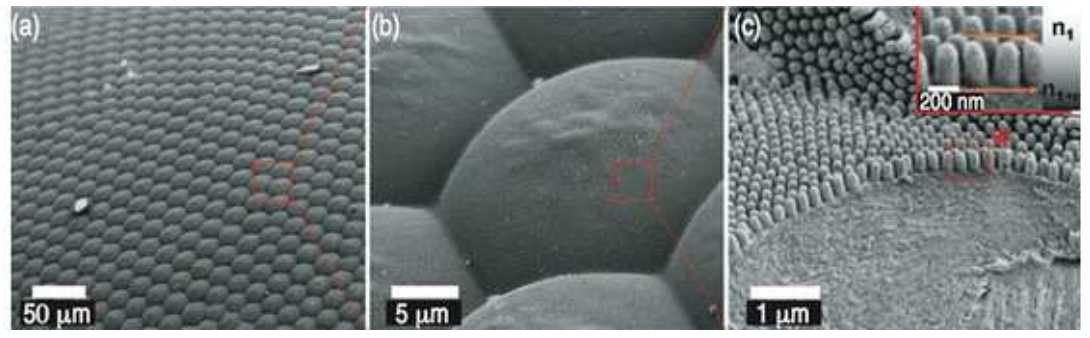

Figure 1.1. Electron microscope image of moth eye at 50 micron, 5 micron, and 1 micron. [5]

\subsection{Solar Cell Operation and Structure}

Solar cells operate by absorption of photons in the solar electromagnetic spectrum in order to create electron-hole pairs, separating those charge carriers in the junction, and collection of the carriers at the terminals to drive a direct electric current [6]. Photons must be of sufficient energy to be absorbed. The energy is used to promote an electron from the valence band to the conduction band and drive the circuit. Photons of energy less than the minimum needed to excite an electron across the energy gap are reflected or transmitted by the cell. Photons of excess energy promote electrons, as well as holes, but this excess energy is lost in the form of heat due to electronic relaxation to the conduction band edge and holes to the valence band edge. Electronic conduction band promotion also creates the absence of an electron, or a hole, at the valence band. The physical 
interpretation of this process has the electron and hole collected at the contact terminals of the device where they enter the circuit [3].

The most basic solar cell structure is the combination of two different types of semiconductors called a p-n junction which forms an interface through which charges interact. The different types can be made of the same original material; typically silicon is used for commercial grade cells. When the two parts are created from a single material, the n-type portion is doped with donor impurities to yield a high electron conductivity in this region. Atoms with more valence electrons than silicon (Group V elements), which has four valence electrons, are used. The p-type portion is made from doping the substance with acceptor ions, those with less valence electrons (Group III elements) than the original semiconductor. This allows for a high hole conductivity in this region [3]. The dopants create a junction with a built-in potential, eliminate the need for a bias voltage, and adjust the valence and conduction band energy levels. This asymmetry is the basic requirement for photovoltaic energy conversion in a solar cell [6]. The Fermi energy, the energy at which the probability of electron occupation is exactly one-half, is split into two quasi-Fermi levels, one for electrons and one for holes. This describes the illuminated state of the cell. The difference between the quasi-Fermi energies corresponds approximately to the output voltage, while the output current can be calculated from the number of absorbed photons and their quantum efficiency [6]. The charge carrier separation occurs in the depletion region, with typical width $\sim 1$ micrometer while the absorption process extends over the whole thickness of 200 micrometer. This 
absorber layer is approximately 150 to 250 microns thick [7]. This is a large amount of material and contributes significantly to the cost of modules.

\subsection{Efficiency Limitations and Design Improvements}

Design improvements create better functioning solar power devices. There are two types of limitations which adversely affect solar cells, intrinsic and extrinsic limitations. Photovoltaic devices can be improved in a few select ways in order to overcome their extrinsic limitations. The intrinsic limitations cannot be overcome by design. They include incoming light energy limitations due to the range of the solar spectrum, Auger recombination, free carrier absorption, and radiative recombination [8,9]. Extrinsic limitations can be overcome and include surface recombination, contact shadowing, series resistance, incomplete collection of photo-generated carriers, and reflection at the front surface, among others [10]. While all of these are important, without allowing the

light to enter the cell, none of the other extrinsic limitations factor into cell performance. Therefore, reflection at the front surface is the first and most basic problem to overcome in cell design.

\subsection{Reflection Minimization}

The design of efficient photovoltaic devices requires the limiting, or eliminating, of power conversion loss mechanisms, including reflection of incoming light, at the front 
surface. Reflection occurs when light travels from a medium to another medium with differing index of refraction. For a solar cell, three different types of incident radiation reflection occur including reflections from contacts, back surfaces, and front surfaces as seen in figure 1.2. The topic of this thesis will focus on front surface reflection minimization.

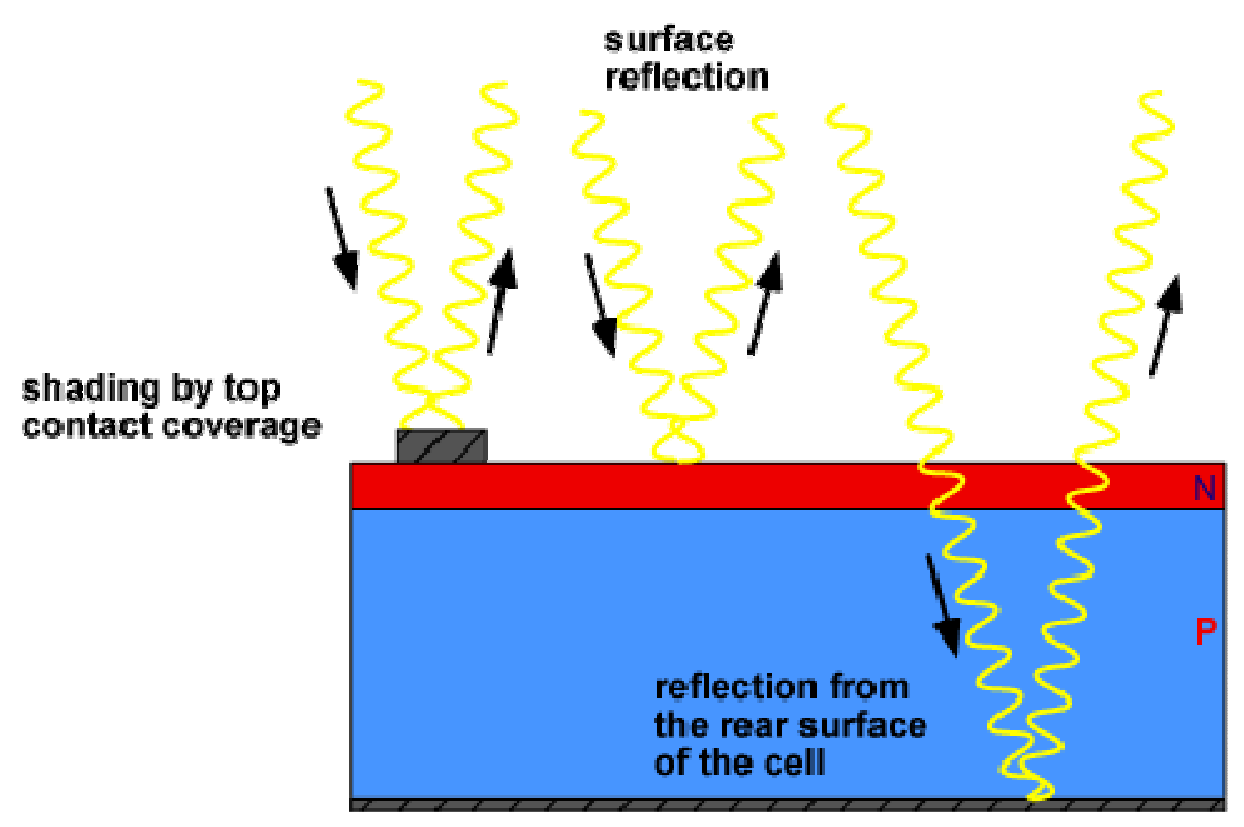

Figure 1.2. Reflection of incident light on a solar cell. [11]

The methods employed to limit reflectivity in solar cells are texturing of the surface and applying planar antireflective coatings. Texturing is used to increase scattering into the cell, which increases the probability of capturing a photon from the incident light, and light trapping which lengthens the path length and increases the probability of electronhole pair production from photon absorption. Planar coatings produce destructive interference with reflected waves and decrease the refractive index of the medium on which the light is incident. 
The employment of antireflective coatings has greatly diminished this problem [12]. By using a thickness equal to one-quarter times the incident wavelength, reflections can be minimized. However, these coatings are for a single wavelength and must be applied in successive layers for broadband effects. The planar coatings do not take into account the different angles at which light can be incident [13]. Successive layering of individually antireflective coatings will aid performance of a cell but also increases manufacturing costs and difficulty.

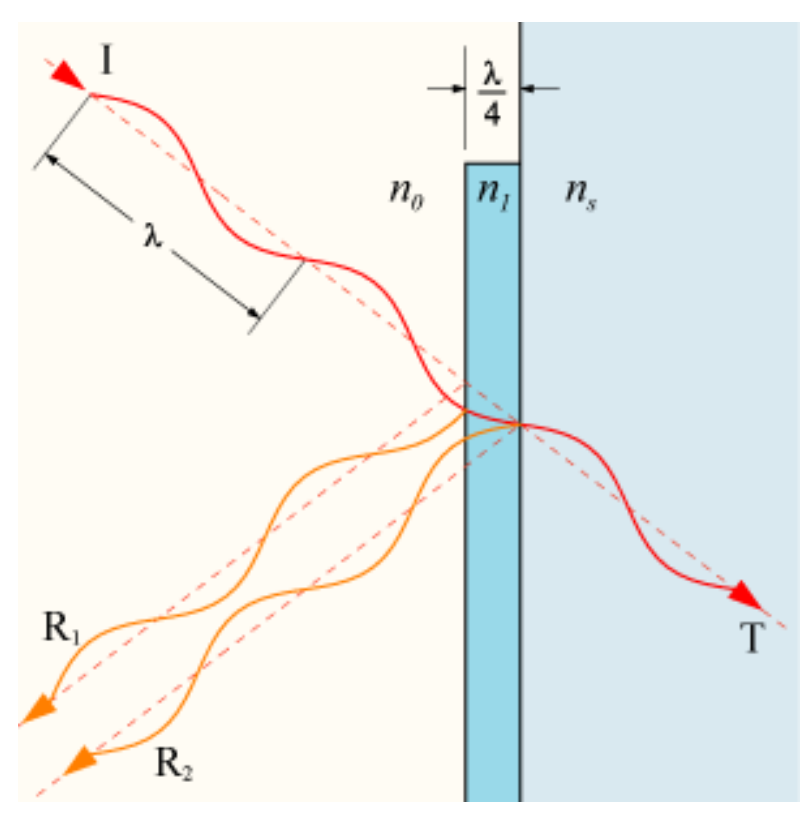

Figure 1.3. Single planar AR coating showing destructive interference of the first and second reflected waves $R_{1}$ and $R_{2}$ due to the quarter-wave phase difference. The thickness of the coating is $d=m \frac{\lambda}{4}$ where $m$ is an positive integer, the indices of refraction for the incident, AR layer, and substrate are $n_{0}, n_{1}$, and $n_{s}$. [14]

\subsection{Planar Coatings}

Planar anti-reflection coatings are deposited at a depth of one-quarter of the wavelength of incoming light. For each wavelength, there must be an additional coating to minimize 
reflection. Since this is not possible in practice, a few different planar surfaces are used to minimize reflection at the wavelengths associated with maximum intensity in the incident spectrum. From the Fresnel equation for reflectivity, the ideal index of refraction is for an anti-reflection coating can be found. At normal incidence, with a single AR coating of index $n_{1}$, the Fresnel equation, from the field boundary conditions for three media with indices of refraction for the incident and substrate media $n_{0}$ and $n_{2}$, takes the form [13]:

$$
R=\left|\frac{\left(r_{01}+r_{12}\right)^{2}-4 r_{01} r_{12} \sin ^{2}\left(\frac{2 \pi d}{\lambda}\right)}{\left(1+r_{01} r_{12}\right)^{2}-4 r_{01} r_{12} \sin ^{2}\left(\frac{2 \pi d}{\lambda}\right)}\right|
$$

Where $d$ is the AR layer thickness, $\lambda$ is the incident wavelength, and $r_{01}$ and $r_{12}$ are:

$$
r_{01}=\frac{n_{0}-n_{1}}{n_{0}+n_{1}} \text { and } r_{12}=\frac{n_{1}-n_{2}}{n_{1}+n_{2}}
$$

With a thickness of a quarter-wavelength, the sine function terms in the numerator and denominator are zero:

$$
\sin ^{2}\left(\frac{2 \pi}{\lambda} d\right)=\sin ^{2}\left(\frac{2 \pi}{\lambda} * \frac{\lambda}{4}\right)=\sin ^{2}\left(\frac{\pi}{2}\right)=0
$$

The reflection equation is minimized when $R=0$ and is now:

$$
R=\left|\frac{\left(r_{01}+r_{12}\right)^{2}}{\left(1+r_{01} r_{12}\right)^{2}}\right|=\left|\frac{\frac{n_{0}-n_{1}}{n_{0}+n_{1}}+\frac{n_{1}-n_{2}}{n_{1}+n_{2}}}{1+\left(\frac{n_{0}-n_{1}}{n_{0}+n_{1}}\right)\left(\frac{n_{1}-n_{2}}{n_{1}+n_{2}}\right)}\right|^{2}
$$




$$
\begin{aligned}
& =\left|\frac{\frac{\left(2 n_{0} n_{1}-2 n_{1}^{2}\right)}{\left(n_{0}+n_{1}\right)\left(n_{1}+n_{2}\right)}}{\frac{\left(n_{0}+n_{1}\right)\left(n_{1}+n_{2}\right)+\left(n_{0}-n_{1}\right)\left(n_{1}-n_{2}\right)}{\left(n_{0}+n_{1}\right)\left(n_{1}+n_{2}\right)}}\right|^{2} \\
& =\left|\frac{\left(2 n_{0} n_{1}-2 n_{1}^{2}\right)}{\left(n_{0}+n_{1}\right)\left(n_{1}+n_{2}\right)+\left(n_{0}-n_{1}\right)\left(n_{1}-n_{2}\right)}\right|^{2}=0
\end{aligned}
$$

Which yields:

$$
n_{A R}=n_{1}=\sqrt{n_{0} n_{2}}
$$

Therefore, to minimize reflection from a planar surface, it must be coated with a coating of thickness equal to one-quarter the size of the incident wavelength and have an index of refraction equal to the geometric mean of the media which it separates.

\subsection{Textured Antireflection}

An omni-directional, antireflection scheme is desirable to cover a broadband of the electromagnetic spectrum for varying angles of incidence. This can be achieved with texturing of the surfaces. Texturing changes the entrance angle as light enters the cell and extends the optical path length which allows for increased chances of absorption (figure 1.4). This can also be achieved by deposition of tapered nanostructures onto cells. Since these structures can be readily deposited from low-cost materials and utilizing simple technology, nanostructures are considered cost-effective and results-enhancing ways to maximize the efficiency of solar cells [15]. 


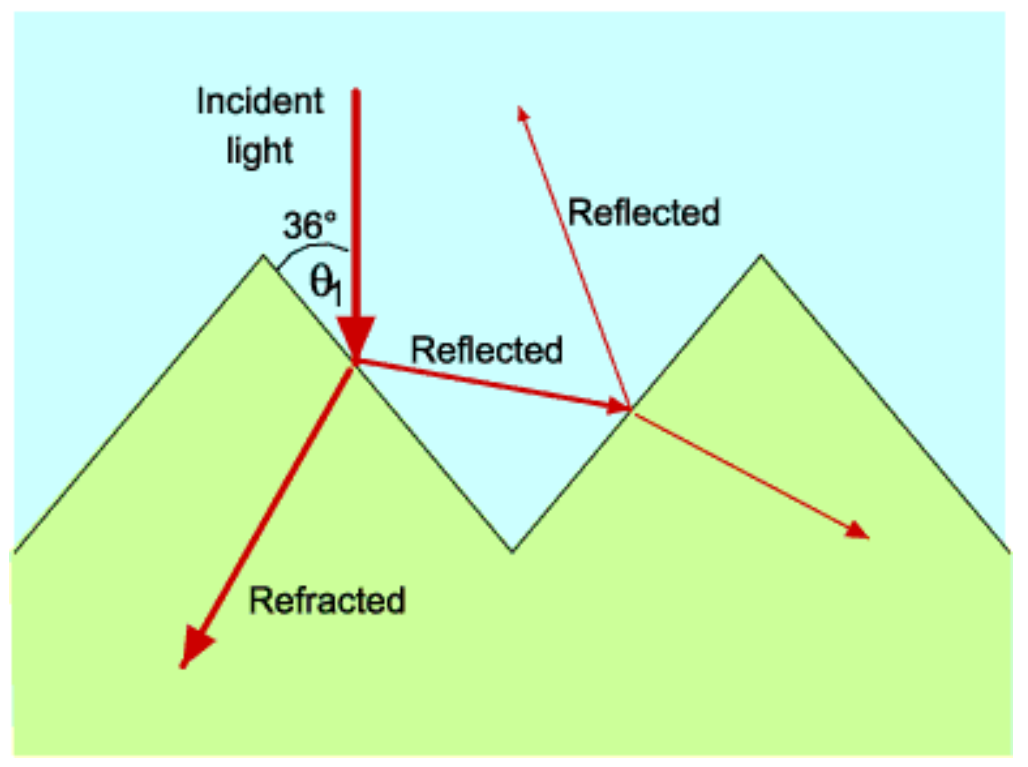

Figure 1.4. Light trapping on textured surface. [16]

\subsection{Zinc Oxide Nanocones as Antireflection Materials}

For standard silicon used in photovoltaic devices, with an index varying between 3.73 to 5.57 in the visible spectrum, the geometric mean, with air as the first medium, ranges from 1.924 to 2.345 [17]. This is the range of zinc oxide's index of refraction [17]. Thus, zinc oxide is a natural choice for a planar anti-reflection coating between air and silicon media because of its index and the fact it is a transparent semiconducting material. Of particular importance here, the growth mechanisms for zinc oxide allow for optimization of the morphology, including tapering of the structures to act as a grading of the refractive index [18]. The nanostructures can be grown as $\mathrm{ZnO}$ nanowires and then tapered to form nanocone shapes. The nanowire radius, height, density, and tilt, with respect to the z-axis, can be controlled by growth conditions. This allows for customization of the morphology to achieve the desired results [19]. In addition to the 
performance properties which make $\mathrm{ZnO}$ a good choice, the inert qualities of $\mathrm{ZnO}$ and its abundance and low cost allow for ease of implementation in manufacturing.

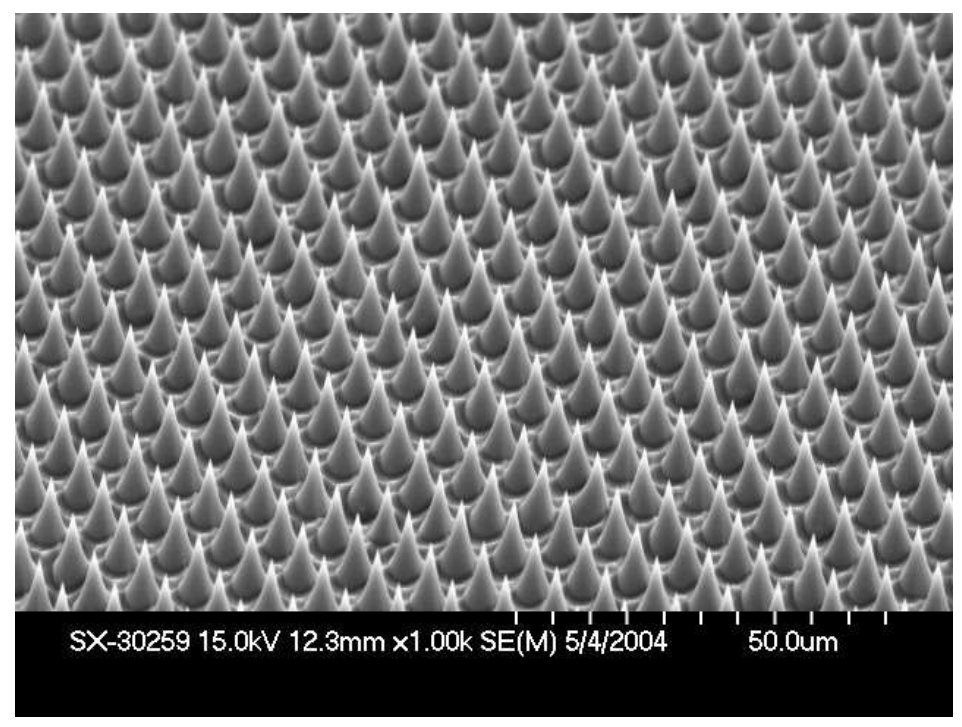

Figure 1.5. Nanocones constructed at Oak Ridge National Laboratory. [20]

\subsection{Motivation for Computation and Effective Medium Approximation}

In the past, modeling for multiple AR coating structures were calculated using transfer matrix method and much of the modeling for structured interfaces was accomplished by applying the effective medium approximation [21]. This can be useful for some 2dimensional geometry, however, limitations arise which cause the need to use a directsolving method applied to the Maxwell equations.

The effective medium approximation is used to model interactions between two media, which have inherent inhomogeneity, as a homogeneous mixture with an associated effective dielectric function or refractive index [22]. The refractive index for the first medium is $n_{1}$ and the second medium is $n_{2}$. The volume filled by the $\mathrm{ZnO}$ is $f_{2}$ and the 
by the air is $f_{1}=1-f_{2}$. The generalized effective medium equation for two different media, in terms of the refractive indices, is:

$$
f_{2} \frac{n_{2}^{2}-n_{e f f}^{2}}{n_{2}^{2}+2 n_{e f f}^{2}}+\left(1-f_{2}\right) \frac{n_{1}^{2}-n_{e f f}^{2}}{n_{1}^{2}+2 n_{e f f}^{2}}=0
$$

For use with zinc oxide structures in air, the equation becomes:

$$
f_{Z n o} \frac{n_{Z n o}^{2}-n_{e f f}^{2}}{n_{Z n o}^{2}+2 n_{e f f}^{2}}+\left(1-f_{Z n o}\right) \frac{n_{\text {air }}^{2}-n_{e f f}^{2}}{n_{\text {air }}^{2}+2 n_{e f f}^{2}}=0
$$

Here, $n_{e f f}$ is the effective index of refraction for individual slabs and $f_{Z n O}$ is the percentage of zinc oxide which fills the volume.

The nanostructure models created contain radii and separation distances significantly smaller than the incident light wavelengths. This leads to optical properties which are driven by multiple diffuse scattering events [23]. Due to the effective medium theory's convergence issues when calculating solutions for high-scattering models the approximation cannot accurately model the nanocone-field interactions. Despite the recent formulation of a transfer matrix model for $n$-scatters using a multipole expansion, COMSOL's direct solving of the fields at the nodal points allows for a more accurate description of the field responses [22]. The direct solving of Maxwell's equations using finite elements also solves random geometry, such as a tilt in the structure, without the need to approximate the system as a single body. Directly solving the equations also allows for a near-field solution of the system. This method uses intensity integrations 
over the top portion of the geometry which encompasses the backscattered light [24]. With the subtraction of the incident intensity, the reflection can be calculated.

\subsection{Thesis and Research Objectives}

Previous laboratory work has shown a decrease in reflectance when employing a nanostructured layer on the front surface of silicon substrates. This decrease was evident for a varying incident angle and wavelength of light. This study was undertaken to recreate a similar model using a direct solver to simulate the reflection minimization by $\mathrm{ZnO}$ nanostructures on $\mathrm{Si}$ substrates. The structure for the laboratory synthesized nanowires (figure 1.6) differed from the cones in this study.

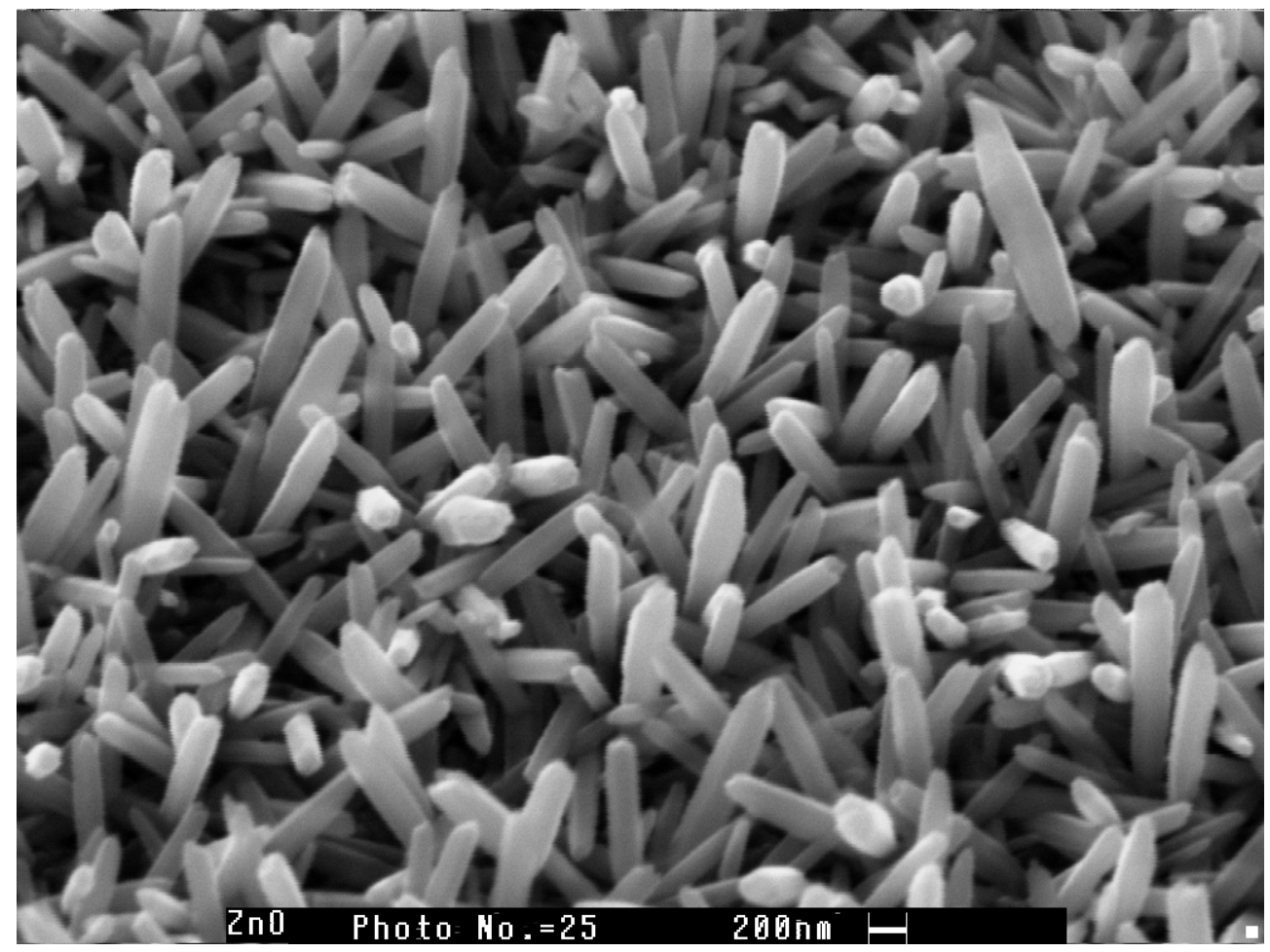

Figure 1.6. Lab grown ZnO nanowires on conductive oxide substrate. [25] 
Greater angles of incline from base-to-tip were observed, with some nearly parallel with the substrate. This was neglected here due to computational difficulties. Also, the wires did not have large tapering at the top and were Wurzite extrusions grown from the substrate. The differing geometries are significant, nevertheless, the laboratory experience prompted this study to utilize nanostructures to show a decrease of total reflection from silicon substrates.

COMSOL Multiphysics® was used to model the nanocones and obtain reflectance values at varying angles and wavelengths. The data is then compared with the ideal values, as calculated from quintic gradient index profile media, and the laboratory plots. A decrease in reflectivity is obtained in the models by addition of conical structures. 


\section{Theory}

\subsection{Fresnel Equations, Plane Waves, and Maxwell's Equations}

The incident light on a solar cell is governed by Maxwell's equations of electrodynamics. The generalized equations for the electric and magnetic fields in terms of the electric

field $\vec{E}$, displacement vector $\vec{D}$, and the magnetic fields in vacuum and medium, $\vec{B}$ and $\vec{H}$ respectively are [26]:

$$
\begin{gathered}
\vec{\nabla} \cdot \vec{D}=\rho_{f} \\
\vec{\nabla} \times \vec{E}=-\frac{\partial \vec{B}}{\partial t} \\
\vec{\nabla} \cdot \vec{B}=0 \\
\vec{\nabla} \times \vec{H}=\overrightarrow{J_{f}}+\frac{\partial \vec{D}}{\partial t}
\end{gathered}
$$

It is often more convenient to use Maxwell's equations in terms of only two vector fields, the electric and the magnetic field, $\vec{E}$ and $\vec{B}$. This can be accomplished by applying the constitutive relations. These relations express $\vec{D}$ in terms of vector $\vec{E}$ and the polarization vector $\vec{P}$, and $\vec{B}$ in terms of $\vec{H}$ and the magnetization $\vec{M}$ [26]:

$$
\begin{gathered}
\vec{D}=\epsilon_{0} \vec{E}+\vec{P}=\epsilon \vec{E} \\
\vec{H}=\mu_{0}{ }^{-1} \vec{B}-\vec{M}=\mu \vec{B} \\
\overrightarrow{J_{f}}=\sigma \vec{E}
\end{gathered}
$$


Here $\epsilon_{0}$ and $\mu_{0}$ are the permittivity and permeability of free space and $\varepsilon$ and $\mu$ are the permittivity and permeability of the materials, while $\sigma$ is the conductivity. For linear media, the polarization and magnetization are directly proportional to the $\vec{E}$ and $\vec{B}$ fields so the $\vec{P}$ and $\vec{M}$ vectors can be absorbed into the $\vec{E}$ and $\vec{B}$ vectors. Now $\varepsilon$ and $\mu$ can be considered constants. For a non-conductive system, such as those considered in the models and containing no sources, the charge density $\rho_{f}$ and current density $J_{f}$ are equal to zero. Putting this together with the previous relations and equations yields:

$$
\begin{gathered}
\vec{\nabla} \cdot \vec{E}=0 \\
\vec{\nabla} \times \vec{E}=-\frac{\partial \vec{B}}{\partial t} \\
\vec{\nabla} \cdot \vec{B}=0 \\
\vec{\nabla} \times \vec{B}=\mu \epsilon \frac{\partial \vec{E}}{\partial t}
\end{gathered}
$$

These are the more familiar, source-less Maxwell equations. Since the light from the visible spectrum is to be considered, the electromagnetic wave equations are useful in determining the field response over time. Using the above form of Maxwell's equations, one can eliminate some equations to get a single vector quantity of interest. Taking the curl of the second equation, and using the vector triple product identity yields:

$$
\begin{gathered}
\vec{\nabla} \times \vec{\nabla} \times \vec{E}=\vec{\nabla}(\vec{\nabla} \cdot \vec{E})-\nabla^{2} \vec{E}=-\nabla^{2} \vec{E}=\vec{\nabla} \times\left(-\frac{\partial \vec{B}}{\partial t}\right)=-\frac{\partial}{\partial t} \vec{\nabla} \times \vec{B} \\
-\nabla^{2} \vec{E}=-\frac{\partial}{\partial t} \vec{\nabla} \times \vec{B}=-\frac{\partial}{\partial t}\left(\mu \epsilon \frac{\partial \vec{E}}{\partial t}\right)
\end{gathered}
$$




$$
\nabla^{2} \vec{E}-\mu \epsilon \frac{\partial^{2} \vec{E}}{\partial t^{2}}=0
$$

Similarly,

$$
\begin{gathered}
\vec{\nabla} \times \vec{B}=\vec{\nabla}(\vec{\nabla} \cdot \vec{B})-\nabla^{2} \vec{B}=-\nabla^{2} \vec{B}=\vec{\nabla} \times \mu \epsilon \frac{\partial \vec{E}}{\partial t}=\mu \epsilon \frac{\partial}{\partial t} \vec{\nabla} \times \vec{E} \\
-\nabla^{2} \vec{B}=\mu \epsilon \frac{\partial}{\partial t} \vec{\nabla} \times \vec{E}=\mu \epsilon \frac{\partial}{\partial t}\left(-\frac{\partial \vec{B}}{\partial t}\right) \\
\nabla^{2} \vec{B}-\mu \epsilon \frac{\partial^{2} \vec{B}}{\partial t^{2}}=0
\end{gathered}
$$

Introducing the wave speed, which is the speed of light $c$ in a vacuum,

$$
c^{2}=\frac{1}{\mu \epsilon}=\frac{1}{\mu_{0} \epsilon_{0}}(\text { in vacuum })
$$

gives one the electromagnetic form of the wave equation, with twice-differentiated vectors with respect to space and time and the squared wave speed as a pre-factor:

$$
\begin{aligned}
& \nabla^{2} \vec{E}-\frac{1}{c^{2}} \frac{\partial^{2} \vec{E}}{\partial t^{2}}=0 \\
& \nabla^{2} \vec{B}-\frac{1}{c^{2}} \frac{\partial^{2} \vec{B}}{\partial t^{2}}=0
\end{aligned}
$$

\subsection{Plane Waves}

For an incoming plane wave, representing light from a distance source, for instance the sun, the equations can be modified to allow for time-harmonic fields, or those with 
sinusoidal variation. The fields can be rewritten to include an amplitude with spatiallyand time-varying portions [27]:

$$
\begin{aligned}
& \vec{E}(\vec{r}, t)=\vec{E}_{0} e^{i(\vec{k} \cdot \vec{r}-\omega t)} \\
& \vec{B}(\vec{r}, t)=\vec{B}_{0} e^{i(\vec{k} \cdot \vec{r}-\omega t)}
\end{aligned}
$$

$\vec{E}_{0}$ and $\vec{B}_{0}$ are the wave amplitudes, and can be complex vectors in general, $\vec{r}$ is the position vector, $\vec{k}$ is the wave vector, and $\omega$ is the angular frequency. The amplitudes need only to be considered constant and real for these purposes. Applying the new timeharmonic fields to the electromagnetic wave equations gives:

$$
\begin{gathered}
\nabla^{2} \vec{E}(\vec{r}, t)-\frac{1}{c^{2}} \frac{\partial^{2} \vec{E}(\vec{r}, t)}{\partial t^{2}}=\nabla^{2} \vec{E}_{0} e^{i(\vec{k} \cdot \vec{r}-\omega t)}-\frac{1}{c^{2}} \frac{\partial^{2} \vec{E}_{0} e^{i(\vec{k} \cdot \vec{r}-\omega t)}}{\partial t^{2}}=0 \\
\nabla^{2} \vec{E}_{0} e^{i(\vec{k} \cdot \vec{r}-\omega t)}-\frac{1}{c^{2}} i^{2}(-\omega)^{2} \vec{E}_{0} e^{i(\vec{k} \cdot \vec{r}-\omega t)}=\nabla^{2} \vec{E}_{0} e^{i(\vec{k} \cdot \vec{r}-\omega t)}-\left(\frac{\omega}{c}\right)^{2} \vec{E}_{0} e^{i(\vec{k} \cdot \vec{r}-\omega t)}=0 \\
\nabla^{2} \vec{E}_{0} e^{i(\vec{k} \cdot \vec{r}-\omega t)}-k^{2} \vec{E}_{0} e^{i(\vec{k} \cdot \vec{r}-\omega t)}=\nabla^{2} \vec{E}(\vec{r}, t)-k^{2} \vec{E}(\vec{r}, t)=0
\end{gathered}
$$

Similarly, the $\vec{B}$-field response can be formulated in the same way:

$$
\begin{gathered}
\nabla^{2} \vec{B}(\vec{r}, t)-\frac{1}{c^{2}} \frac{\partial^{2} \vec{B}(\vec{r}, t)}{\partial t^{2}}=\nabla^{2} \vec{B}_{0} e^{i(\vec{k} \cdot \vec{r}-\omega t)}-\frac{1}{c^{2}} \frac{\partial^{2} \vec{B}_{0} e^{i(\vec{k} \cdot \vec{r}-\omega t)}}{\partial t^{2}}=0 \\
\nabla^{2} \vec{B}_{0} e^{i(\vec{k} \cdot \vec{r}-\omega t)}-\frac{1}{c^{2}} i^{2}(-\omega)^{2} \vec{B}_{0} e^{i(\vec{k} \cdot \vec{r}-\omega t)}=\nabla^{2} \vec{B}_{0} e^{i(\vec{k} \cdot \vec{r}-\omega t)}-\left(\frac{\omega}{c}\right)^{2} \vec{B}_{0} e^{i(\vec{k} \cdot \vec{r}-\omega t)}=0 \\
\nabla^{2} \vec{B}_{0} e^{i(\vec{k} \cdot \vec{r}-\omega t)}-k^{2} \vec{B}_{0} e^{i(\vec{k} \cdot \vec{r}-\omega t)}=\nabla^{2} \vec{B}(\vec{r}, t)-k^{2} \vec{B}(\vec{r}, t)=0
\end{gathered}
$$


These are the time-harmonic wave equations which are to be solved for the field response. The vector $\vec{k}$ is the wave vector and its magnitude is $|\vec{k}|^{2}=\frac{\omega^{2}}{c^{2}}$. It defines the propagation of the wave [27]. The fields in this form allow for plane wave analysis with a source far enough away to be considered perfectly planar wave fronts impinging on the surface. As light travels to the medium of the device, it is not just in plane wave form, but also has definitive field vectors associated with it and thus phase properties. This incident light will be acted on in two distinct ways when interacting with the medium of the solar cell, if one does not account for absorption; it will be reflected or it will be transmitted. This leads to the Fresnel equations. Figure 2.1 shows the relationship between the incident, reflected, and transmitted fields for a transverse electric wave.

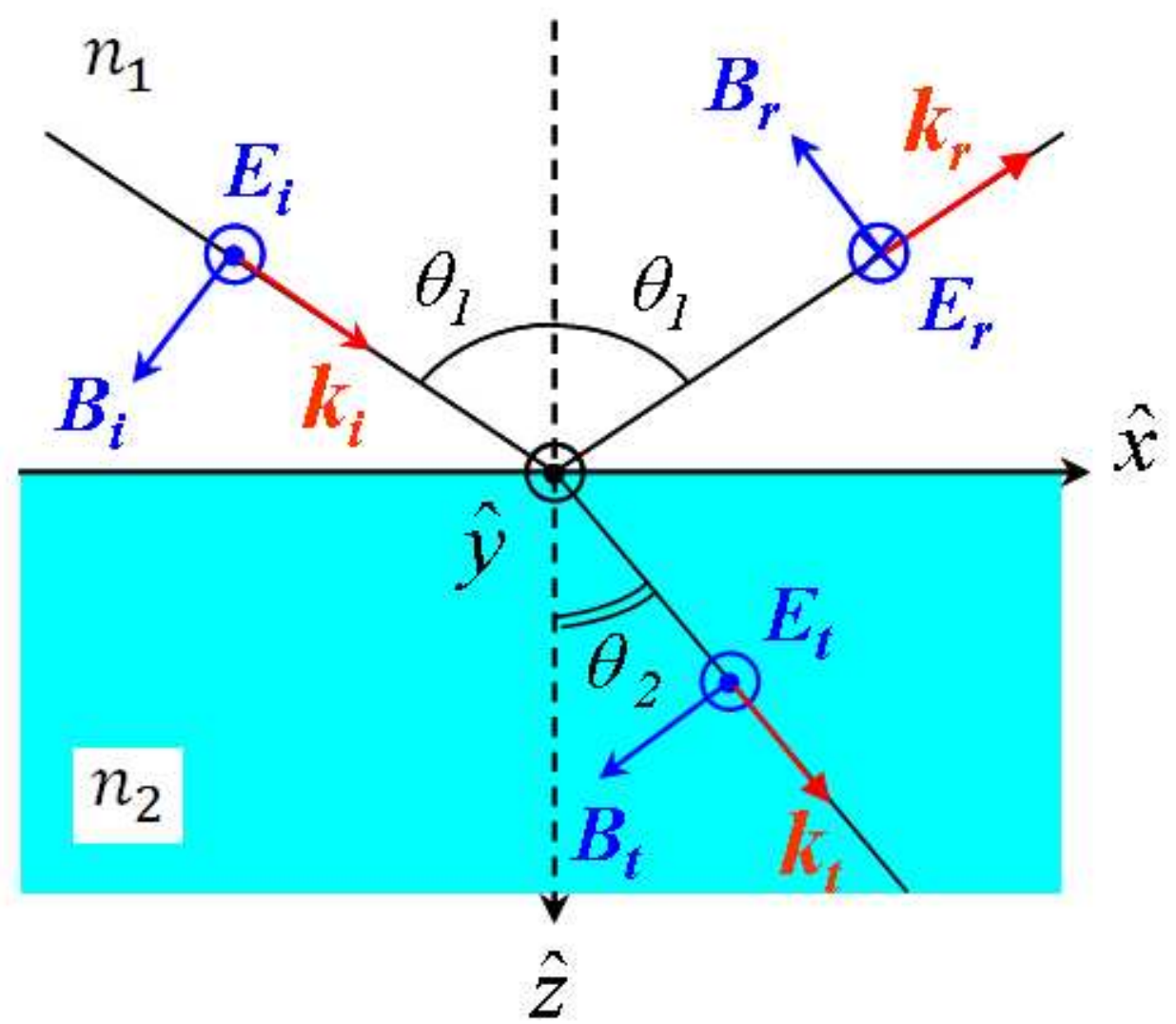

Figure 2.1. Incident, reflected, and transmitted fields for a TE wave. [28] 
The incident electromagnetic wave is transverse, with the electric field, magnetic field, and the wave vector all mutually orthogonal from a medium with index of refraction $n_{1}$ to a medium with index $n_{2}$. The index of refraction of a material is defined as:

$$
n=\sqrt{\frac{\mu \epsilon}{\mu_{0} \epsilon_{0}}}=\sqrt{\mu_{r} \epsilon_{r}}
$$

The index can be put into terms or the relative permeability $\mu_{r}$ and relative permittivity $\epsilon_{r}$ in order to simplify the equation and disregard the unit system [29].

\subsection{Fresnel Equations}

Boundary conditions imposed on electrodynamic fields leads to a relationship between the incident, reflected, and transmitted waves' amplitudes called Fresnel's equations. For media with matching permeabilities, these can be written in terms of the indices of refraction.

Concentrating on the electric field, the complex, exponential forms are altered to distinguish between the different directions. The utilization of time-harmonic electric and magnetic field formulations for incoming, reflected, and transmitted fields yields [26, 27]:

$$
\begin{gathered}
\text { Incident: } \vec{E}_{i}(\vec{r}, t)=\vec{E}_{0_{i}} e^{i\left(\vec{k}_{i} \cdot \vec{r}-\omega_{i} t\right)} \\
\text { Reflected: } \vec{E}_{r}(\vec{r}, t)=\vec{E}_{0_{r}} e^{i\left(\vec{k}_{r} \cdot \vec{r}-\omega_{r} t\right)} \\
\text { Transmitted: } \vec{E}_{t}(\vec{r}, t)=\vec{E}_{0_{t}} e^{i\left(\vec{k}_{t} \cdot \vec{r}-\omega_{t} t\right)}
\end{gathered}
$$


The wave numbers are defined by:

$$
k_{i}^{2}=\left(\frac{n_{1} \omega_{i}}{c}\right)^{2}, k_{r}^{2}=\left(\frac{n_{1} \omega_{r}}{c}\right)^{2}, k_{t}^{2}=\left(\frac{n_{2} \omega_{t}}{c}\right)^{2}
$$

At a given point in the medium from which light is incident, the total field is the sum of the contributions from the incident and reflected fields. In the second medium, where transmission occurs, the total field is just given by the transmitted field. Since the fields are coplanar in nature, and must be continuous across the boundary in which the reflection occurs, the tangential components of the total fields must be equal. Combining this equality with the above formulations of the fields gives:

$$
\left[\vec{E}_{0_{i}} e^{i\left(\vec{k}_{i} \cdot \vec{r}-\omega_{i} t\right)}+\vec{E}_{0_{r}} e^{i\left(\vec{k}_{r} \cdot \vec{r}-\omega_{r} t\right)}\right]_{\text {tang }}=\left[\vec{E}_{0_{t}} e^{i\left(\vec{k}_{t} \cdot \vec{r}-\omega_{t} t\right)}\right]_{\text {tang }}
$$

For this equation to hold true, the exponentials must have equivalent arguments.

Therefore, the frequencies must be the same since the frequency of a monochromatic wave cannot be changed by a reflection from an interface:

$$
\omega_{i}=\omega_{r}=\omega_{t}
$$

The wave vectors dotted into the position vector are equivalent, or all the wave vectors lie in the same plane:

$$
\vec{k}_{i} \cdot \vec{r}=\vec{k}_{r} \cdot \vec{r}=\vec{k}_{t} \cdot \vec{r}
$$

This leads to a relationship between the field amplitudes:

$$
\vec{E}_{0_{i}}+\vec{E}_{0_{r}}=\vec{E}_{0_{t}}
$$


The interaction of light with materials is formulated using the $\vec{E}$ and $\vec{H}$ fields. In the same manner as the electric field boundary relationship is found, the $\vec{H}$ field is found.

$$
H_{1 t}=H_{2 t}
$$

For S-polarization, or transverse electric (TE) mode, the fields have the following orientation near the surface boundary:

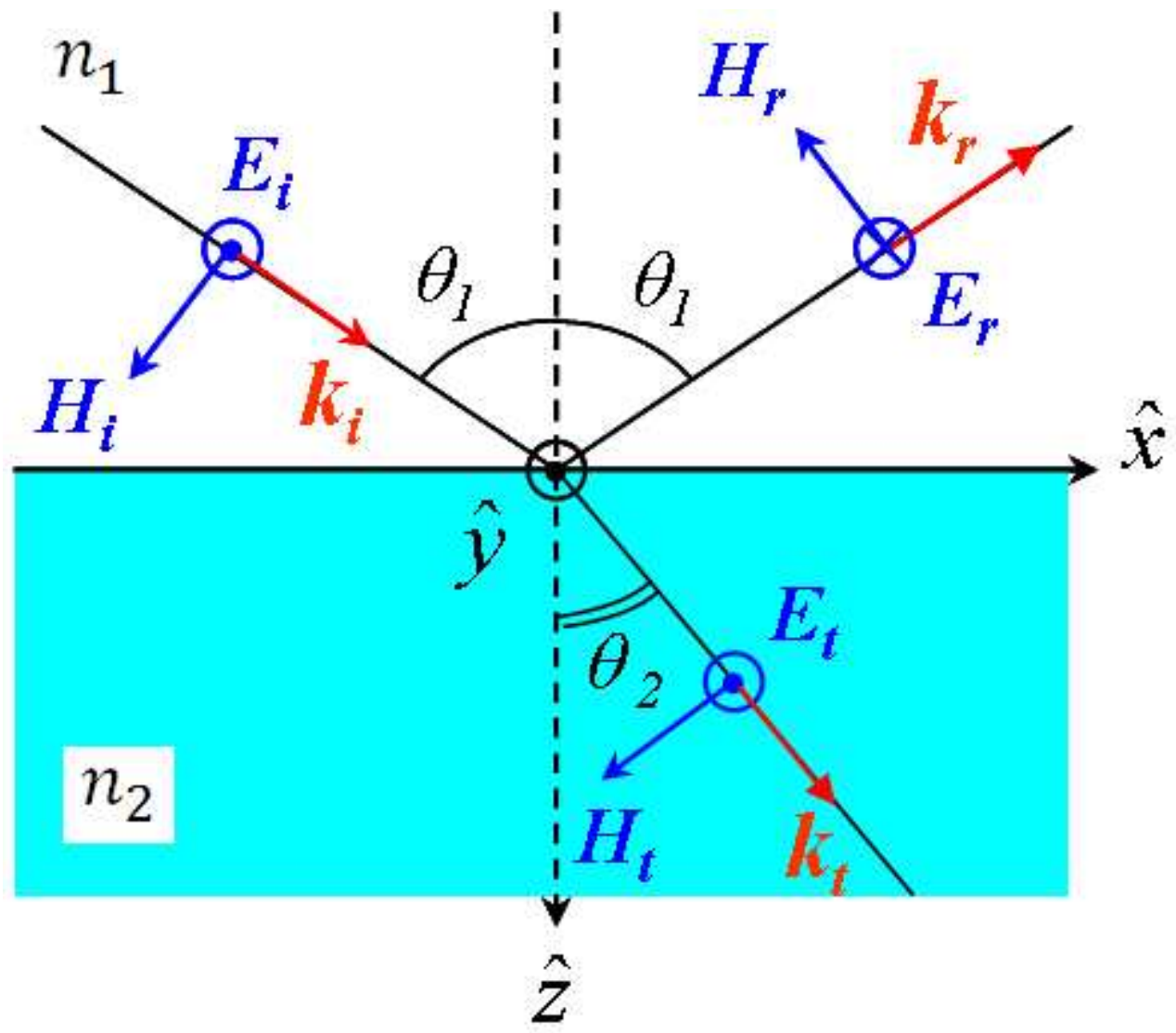

Figure 2.2. Incident, reflected, and transmitted fields for a TE wave. [30]

For figure 2.2, the electric field is in the plane of incidence and has no component in the direction of propagation. Using elementary trigonometry and the tangential component continuity equation: 


$$
\begin{gathered}
H_{0_{i}} \cos \theta_{i}+H_{0_{r}} \cos \theta_{i}=H_{0_{t}} \cos \theta_{t} \\
\text { For } \theta_{1}=\theta_{i}=\theta_{r} \text { and } \theta_{2}=\theta_{t}
\end{gathered}
$$

Defining the impedance for a medium:

$$
Z=\frac{E_{n}}{H_{n}}
$$

Since the ratio of the electric and magnetic fields produces units of ohms, this yields:

$$
\frac{E_{0_{i}}}{Z_{1}} \cos \theta_{i}+\frac{E_{0_{r}}}{Z_{1}} \cos \theta_{i}=\frac{E_{0_{2}}}{Z_{2}} \cos \theta_{t}
$$

Using the above equations, the following ratios are obtained for a TE wave [31]:

$$
\begin{aligned}
& \left(\frac{E_{0_{r}}}{E_{0_{i}}}\right)_{\perp}=\frac{Z_{2} \cos \theta_{i}-Z_{1} \cos \theta_{t}}{Z_{2} \cos \theta_{i}+Z_{1} \cos \theta_{t}} \\
& \left(\frac{E_{0_{t}}}{E_{0_{i}}}\right)_{\perp}=\frac{2 Z_{2} \cos \theta_{i}}{Z_{2} \cos \theta_{i}+Z_{1} \cos \theta_{t}}
\end{aligned}
$$

For P-polarization, or transverse magnetic (TM) mode, the fields have the orientation near the surface boundary seen in figure 2.3 .

For these fields, the orientations are reversed. The tangential components are again equated with the electric field being put into trigonometric component form and the magnetic components subtracted since there is a direction shift upon reflection:

$$
E_{0_{i}} \cos \theta_{i}+E_{0_{r}} \cos \theta_{i}=E_{0_{t}} \cos \theta_{t}
$$




$$
\text { For } \theta_{1}=\theta_{i}=\theta_{r} \text { and } \theta_{2}=\theta_{t}
$$

and

$$
\vec{H}_{0_{i}}-\vec{H}_{0_{r}}=\vec{H}_{0_{t}}
$$

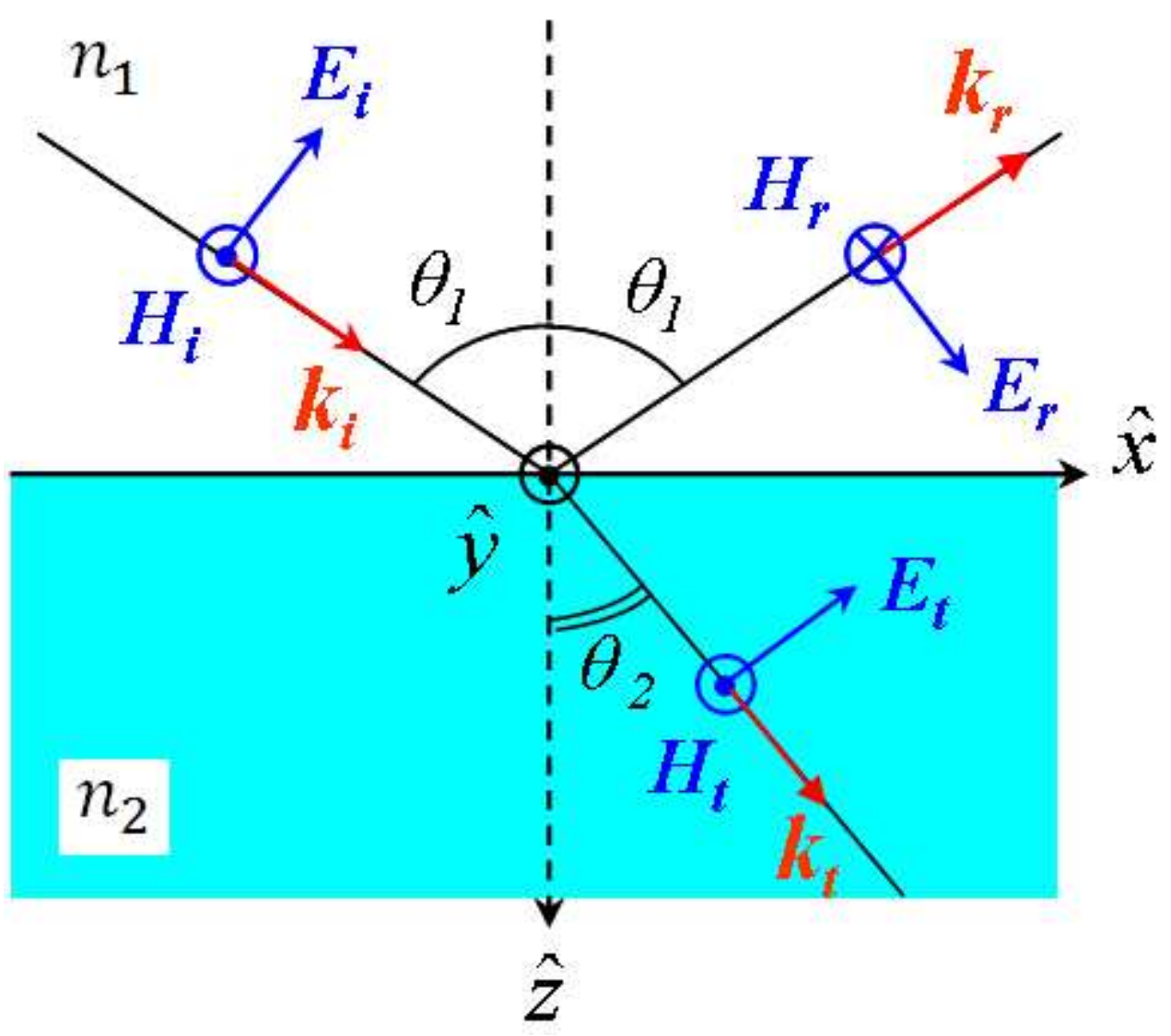

Figure 2.3. Incident, reflected, and transmitted fields for a TM wave. [32]

Again using the wave impedance and similar mathematical substitutions, the following ratios are obtained for a wave with an electric field parallel to the plane of incidence [27]: 


$$
\begin{aligned}
& \left(\frac{E_{0_{r}}}{E_{0_{i}}}\right)_{\|}=\frac{Z_{2} \cos \theta_{t}-Z_{1} \cos \theta_{i}}{Z_{2} \cos \theta_{t}+Z_{1} \cos \theta_{i}} \\
& \left(\frac{E_{0_{t}}}{E_{0_{i}}}\right)_{\|}=\frac{2 Z_{2} \cos \theta_{i}}{Z_{2} \cos \theta_{t}+Z_{1} \cos \theta_{i}}
\end{aligned}
$$

To put the Fresnel equations in terms of the indices of refraction, the impedance is further modified. The case involving two dielectrics is considered. Defining the so-called "impedance of free space" as the ratio of the permeability of free space to the permittivity of free space, the equations can then be modified, for non-conducting, non-magnetic media, for the index-dependent equations [29]:

$$
\begin{gathered}
Z=\frac{E}{H}=\sqrt{\frac{\mu}{\epsilon} \text { and } n}=\sqrt{\mu_{r} \epsilon_{r}} \cong \sqrt{\epsilon_{r}} \\
Z=\sqrt{\frac{\mu}{\epsilon}\left(\frac{\epsilon_{0}}{\epsilon_{0}}\right)\left(\frac{\mu_{0}}{\mu_{0}}\right)}=\sqrt{\frac{\mu}{\mu_{0}}\left(\frac{\epsilon_{0}}{\epsilon}\right)\left(\frac{\mu_{0}}{\epsilon_{0}}\right)}=\sqrt{\frac{\mu}{\mu_{0}}} \frac{Z_{0}}{\sqrt{\epsilon_{r}}}=\sqrt{\frac{\mu}{\mu_{0}}} \frac{Z_{0}}{n} \cong \frac{Z_{0}}{n}
\end{gathered}
$$

Therefore, the reflection and transmission coefficients for TE and TM modes are:

TE Mode $\left\{\begin{array}{l}R=\left|\left(\frac{E_{0_{r}}}{E_{0_{i}}}\right)_{\perp}\right|^{2}=\left|\frac{n_{1} \cos \theta_{i}-n_{2} \cos \theta_{t}}{n_{1} \cos \theta_{i}+n_{2} \cos \theta_{t}}\right|^{2} \\ T=\left|\left(\frac{E_{0_{t}}}{E_{0_{i}}}\right)_{\perp}\right|^{2}=\left|\frac{2 n_{1} \cos \theta_{i}}{n_{1} \cos \theta_{i}+n_{2} \cos \theta_{t}}\right|^{2}\end{array}\right\}$

TM Mode $\left\{\begin{array}{l}R=\left|\left(\frac{E_{0_{r}}}{E_{0_{i}}}\right)_{\|}\right|^{2}=\left|\frac{n_{1} \cos \theta_{t}-n_{2} \cos \theta_{i}}{n_{1} \cos \theta_{t}+n_{2} \cos \theta_{i}}\right|^{2} \\ T=\left|\left(\frac{E_{0_{t}}}{E_{0_{i}}}\right)_{\|}\right|^{2}=\left|\frac{2 n_{1} \cos \theta_{i}}{n_{1} \cos \theta_{t}+n_{2} \cos \theta_{i}}\right|^{2}\end{array}\right\}$ 


\subsection{Conical Profile}

For nanostructured media, the index of refraction is not simply described as in the above discussion. Generally speaking, the index of refraction of a material is a tensor of second rank which has directional dependence. For a linear, isotropic, and homogeneous medium, the index loses spatial variation for an unbounded region. While this type of material is considered here, the isotropy is limited to the interior of the structure. However, a cross-sectional area of the structure displays a mixture of air and nanocone. Therefore, the index of refraction is constant inside the structure but varies for the complete system as a function of the structure's spatial dimensions.

For reflection minimization, the optimal index of refraction for an intermediary between two media of indices equal to $n_{0}$ and $n_{2}$ is:

$$
n_{1}=\sqrt{n_{0} n_{2}}
$$

A material with this index of refraction is deposited at a thickness equal to:

$$
t=\frac{\lambda}{4 n_{1}}
$$

The wavelength of incident light is denoted by $\lambda$. This technique is used in succession for various wavelengths to maximize transmission.

A gradient index of refraction can enhance transmission without the associated thickness requirement. A medium with a graded index has a continuously varying index of refraction which causes light rays travel on curved paths. Gradient media are traditionally 
deposited in layers varying refractive indices or by doping in increasing amounts at increasing depths. Conversely, a graded index can also be realized in layers where the composition is gradually changed with depth. For example, when the air-to-solid volume fraction in a porous film changes with height this film can be considered a graded index material, provided the coarseness of the cones and air gaps are well below the wavelength scale. Tapered nanostructures are a similar case and require only a single deposition to grade the index. Instead of multiple layers of different index, geometric structure provides the grading. For nanocones, a well-defined geometry allows for calculation of the refractive index profile. An air to zinc oxide nanocone interface starts with an index approximately equal to one and slowly varies to equal a fractional portion of zinc oxide as the light travels down the cone. The cone has a height $h$ and a radius $R$. The smaller cone is proportional to the larger and has a height $z$ and radius $r$. The differential portion of the cone with which light is coming into contact is marked as yellow on figure 2.4. For a cube with sides equal to twice the radius of the large cone, and using similar triangles to eliminate the radial dependence, the following mathematical analysis can be performed to find the refractive index profile: 


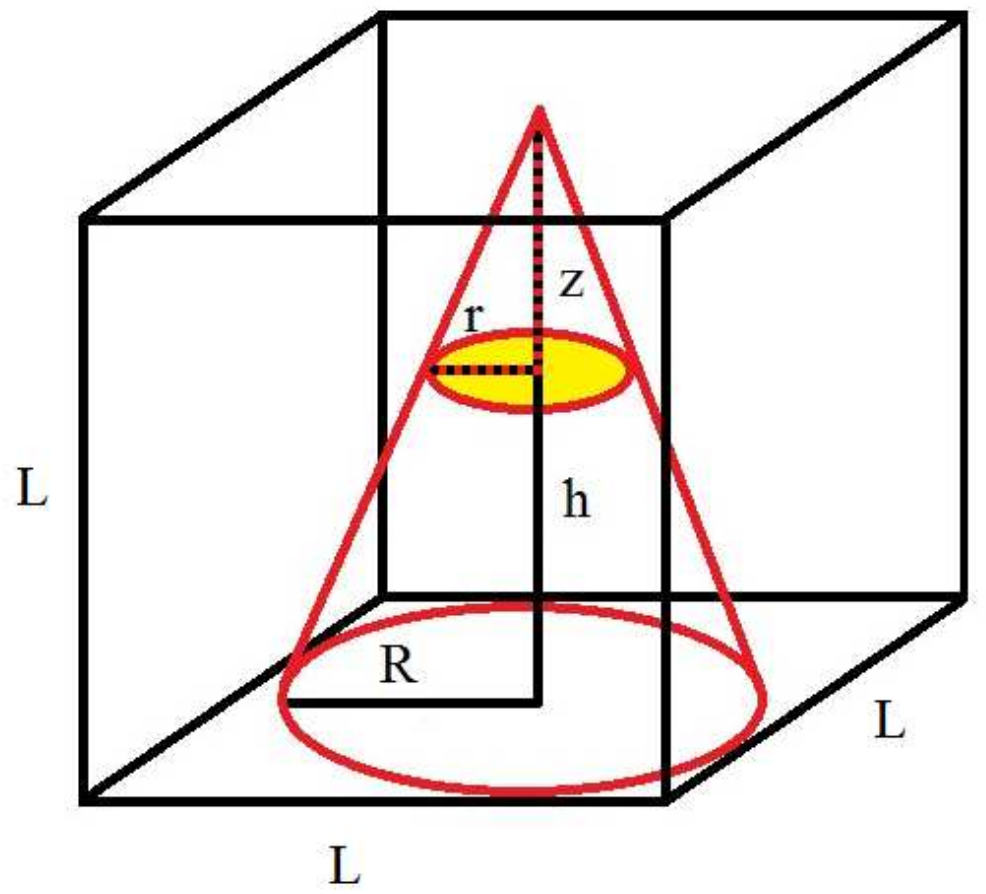

Figure 2.4. Nanocone unit cell. The refractive index is a function of the fractional area of the cone. The cone is consists of air filling with refractive index $n_{0}=1$ and a $\mathrm{ZnO}$ nanocone of index $n_{1}=$ 2. 0516.

Effective refractive index as a function of height:

$$
\begin{gathered}
L^{2} n(z)=L^{2} n_{0}+\left(n_{1}-n_{0}\right) \pi\left(\frac{R}{h}\right)^{2}(h-z)^{2} \\
n(z)=n_{0}+\frac{\left(n_{1}-n_{0}\right) \pi}{L^{2}}\left(\frac{R}{h}\right)^{2}(h-z)^{2} \\
n(z)=n_{0}+\frac{\left(n_{1}-n_{0}\right) \pi}{(2 R)^{2}}\left(\frac{R}{h}\right)^{2}(h-z)^{2} \\
n(z)=n_{0}+\frac{\left(n_{1}-n_{0}\right) \pi}{4 R^{2}}\left(\frac{R^{2}}{h^{2}}\right)(h-z)^{2}
\end{gathered}
$$




$$
n(z)=n_{0}+\left(n_{1}-n_{0}\right) \frac{\pi}{4}\left(\frac{h-z}{h}\right)^{2}
$$

As a check, the profiles of the top and bottom portions of the unit cell can be found. The profile at the top should be equal to the index of air since the extreme peak of the nanocone is infinitesimally small. The bottom of the unit cell should be the area of a square, with refractive index equal to air, and a circular portion in the interior with refractive index equal to zinc oxide.

For $n(z)=n(h)$ :

$$
\begin{aligned}
& n(h)=n_{0}+\left(n_{1}-n_{0}\right) \frac{\pi}{4}\left(\frac{h-h}{h}\right)^{2} \\
& n(h)=n_{0}+\left(n_{1}-n_{0}\right) \frac{\pi}{4}(0)^{2}=n_{0}
\end{aligned}
$$

For $n(z)=n(0)$ :

$$
\begin{gathered}
n(0)=n_{0}+\left(n_{1}-n_{0}\right) \frac{\pi}{4}\left(\frac{h-0}{h}\right)^{2} \\
n(0)=n_{0}+\left(n_{1}-n_{0}\right) \frac{\pi}{4}\left(\frac{h}{h}\right)^{2} \\
n(0)=n_{0}+\left(n_{1}-n_{0}\right) \frac{\pi}{4} \\
n(0)=\left(1-\frac{\pi}{4}\right) n_{0}+\frac{\pi}{4} n_{1}
\end{gathered}
$$

The nanocone has a quadratic refractive index profile. The ideal refractive index profile is quintic in nature: 


$$
n_{\text {quintic }}=n_{0}+\left(n_{1}-n_{0}\right)\left(10\left(\frac{Z}{h}\right)^{3}-15\left(\frac{Z}{h}\right)^{4}+6\left(\frac{Z}{h}\right)^{5}\right)
$$

The nanocone and quintic refractive index profiles are plotted in figure 2.5.

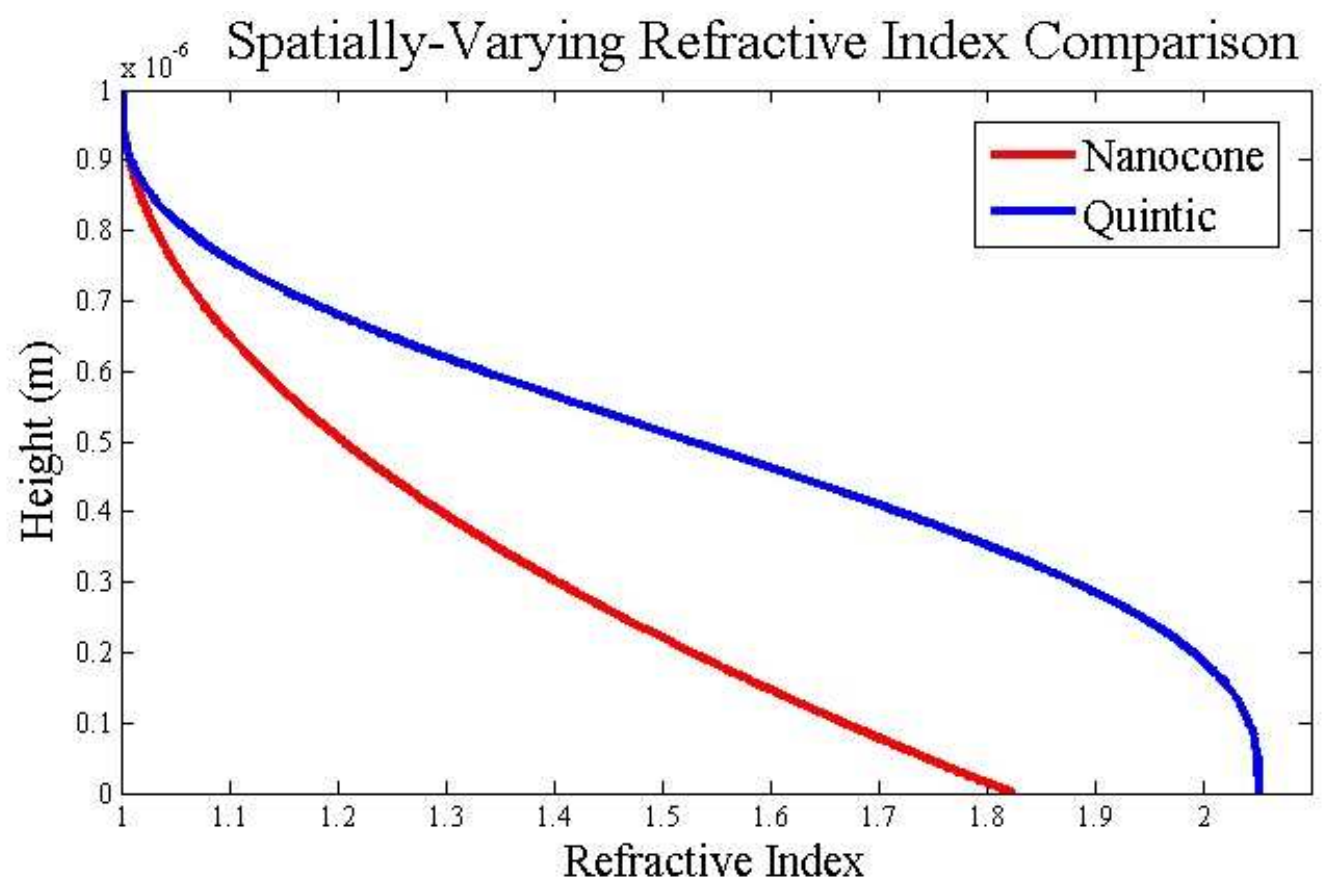

Figure 2.5. Spatially-varying refractive index comparison for nanocones and the quintic profile.

The curves display similar qualities with the exception of an inflection point for the quintic profile. The nanocone profile can be adjusted to contain a similar constant portion of the graph by cascading the cone with a cylindrical base which holds the index profile constant or with a model containing a base layer of $\mathrm{ZnO}$ underneath the cones. Having this layer between the nanocones and the silicon substrate models a system with a seed layer which can be used to enhance nanostructure growth. During laboratory synthesis of nanowire coated silicon, the growth is stimulated with a seed layer deposited before nanostructure growth [19]. This is taken into account in the models and thus alters the 
index profile to behave more like the quintic profile. Figure 2.6 shows the modified profile.

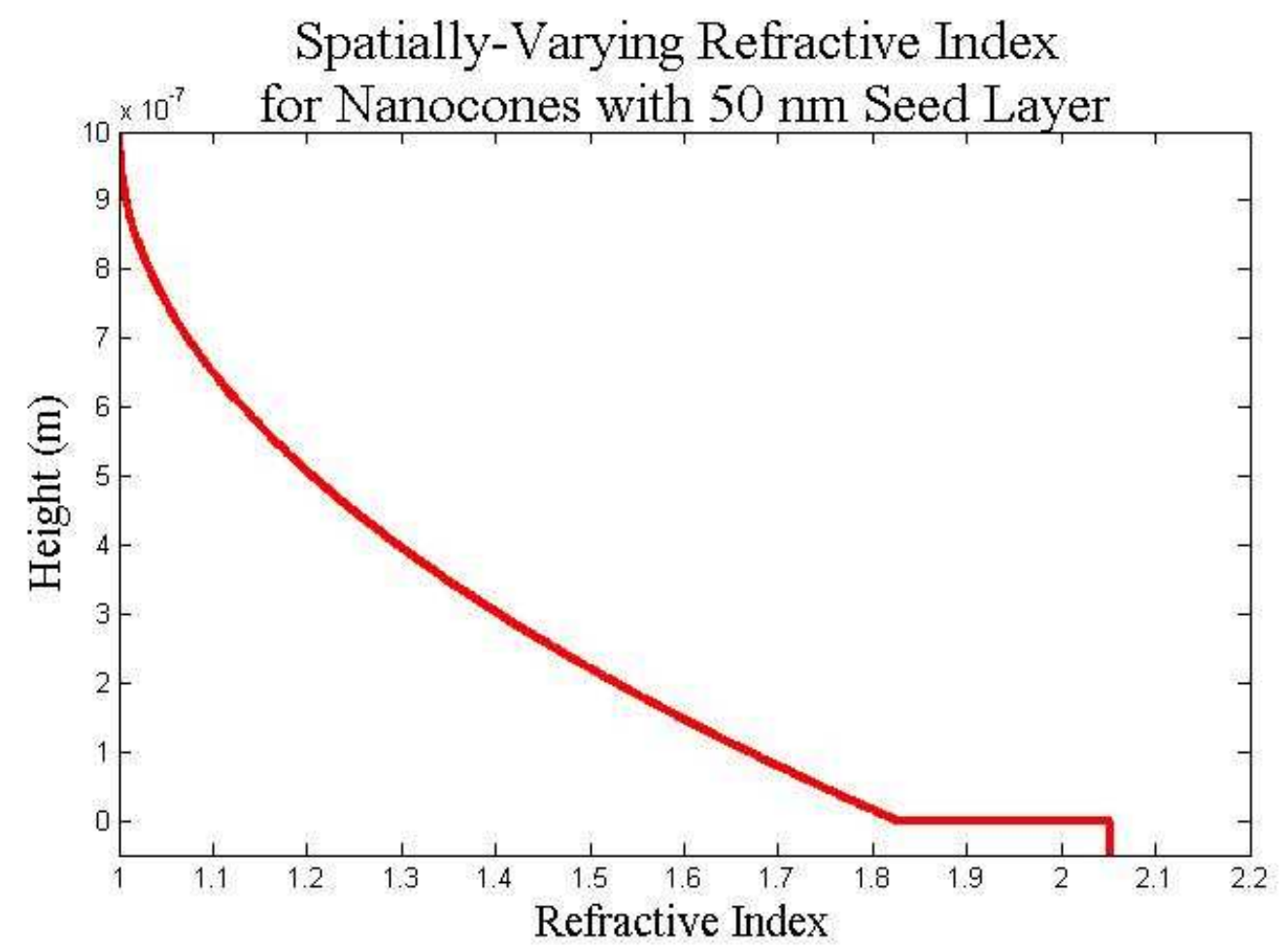

Figure 2.6. Spatially-varying refractive index for nanocones with a seed layer. 


\section{Methods and Computational Model}

\subsection{Computational Equipment}

The large computational power required to accurately model the nanocone-light interactions necessitated access to a system capable of performing the calculations. A 64bit computer was built to handle the simulations utilizing a Windows Enterprise 2008 R2 server. Initially, an Intel Xeon E5506 LGA 1366 Quad-Core Processor was installed, but calculation times needed to be reduced, so a faster eight-core AMD 6212 Opteron processors, clocked at $2.60 \mathrm{GHz}$ each, were installed. A total of $64 \mathrm{~GB}$ of random access memory (RAM) was installed at the outset. However, due to the mesh refinements needed, an additional $112 \mathrm{~GB}$ were added for a total of $176 \mathrm{~GB}$ of RAM. In addition, a one TB hard drive was installed to house the many large files stemming from the models. Having extra storage aided in the computational ability by allowing extra memory to be swapped-in and thus enabling models with larger RAM needs to be computed without shutdowns. The hardware was housed in a single casing. The hardware required to run the models is listed in table 3.1.

\begin{tabular}{|c|c|c|c|c|}
\hline Server & Processor & $\begin{array}{c}\text { Processor } \\
\text { Speed }\end{array}$ & RAM & Memory \\
\hline $\begin{array}{c}\text { Windows } \\
\begin{array}{c}\text { Wnterprise 2008 } \\
\text { R2 }\end{array}\end{array}$ & $\begin{array}{c}\text { AMD 6212 } \\
\text { Opteron } \\
\text { (8 Core) }\end{array}$ & $\begin{array}{c}2.60 \mathrm{GHz} \\
\text { (Dual) }\end{array}$ & $176 \mathrm{~GB}$ & $1 \mathrm{~TB}$ \\
\hline
\end{tabular}

Table 3.1. Computer hardware required for model simulations. 


\subsection{Finite Element Method and PARDISO Solver}

COMSOL operates using finite element analysis. The finite element method (FEM) uses piecewise approximations, in place of, continuous functions. An element which is finite allows a discrete, or digitized, number, relation, or equation to lead to approximate solutions of problems containing analog properties or infinitely large in extent. These replacements are often polynomial in form and enable a finite number of degrees of freedom, in place of a continuum [33].

Physical systems can be described by a governing set of equations and boundary conditions. COMSOL uses FEM to cut the geometry of the model into elements which are linked together with nodes, at which the system solves for the necessary equations. Each node has a unique equation and the set of equations are solved simultaneously for the desired result. The field quantities are interpolated over the elements. All elements adjacent to a particular node have identical degrees of freedom [34].

The system is viewed by finite element analysis software as being approximately linear in nature provided the individual elements are made small enough. At a very small scale, this methodology works satisfactorily in all practical physical systems. However, due to the extremely high number of mesh points, an extremely robust solver must be used. COMSOL uses the PARDISO (parallel sparse direct solver) to compute the solutions to the models. 
PARDISO performs analysis, symbolic and numerical factorization and forward and backward substitution [35]. The PARDISO direct solver calculates the solution to a set of sparse linear equations of the form:

$$
A x=b
$$

This is accomplished by using a parallel Lower-Upper (LU), Lower-Diagonal-Lower (LDL), or $\mathrm{LL}^{\mathrm{T}}$ (Lower-Lower Transpose) factorization [35]. To allow factorization of this kind, the electromagnetic equations must be converted to suitable forms. The purpose of finite element analysis is to construct the matrix A and then solve for the system. This is accomplished by discretization of the fields and geometry. The fields to be discretized by COMSOL are displayed on the graphical user interface with the appearance of the wave equation:

$$
\vec{\nabla} \times\left(\mu^{-1} \vec{\nabla} \times \vec{E}\right)-k_{0}^{2}\left(\epsilon_{r}-\frac{i \sigma}{\omega \epsilon_{0}}\right) \vec{E}=\vec{\nabla} \times\left(\mu^{-1} \vec{\nabla} \times \vec{E}\right)-k_{0}^{2} \epsilon_{r} \vec{E}=\overrightarrow{0}
$$

However, the weak form of the vector wave equation is used which reduces the rigid requirements of an exact solution, provided the modified equation holds true for certain test functions. This approximate solution allows for the use of numerical methods to solve the equations. The weak form of the above wave equation in terms of a test function $W$ is:

$$
\int_{V} \mu^{-1}(\vec{\nabla} \times \vec{E}) \cdot(\vec{\nabla} \times \vec{W}) d V-k_{0}^{2} \int_{V} \epsilon_{r} \vec{E} \cdot \vec{W} d V=0
$$


Vector elements $(\vec{W})$ are used to discretize the field and to satisfy the divergence condition. Scalar functions do not satisfy the divergence condition $\vec{\nabla} \cdot \vec{D}=0$ for the source-less equations since discretization of the electric field makes it globally continuous [36]. Nor do scalar functions enforce continuity in the tangential electric field and the normal displacement field. The field elements take the form:

$$
\vec{E}(\vec{r})=\sum_{n} E_{n} \vec{W}_{n}(\vec{r})
$$

This approach satisfies the above requirements since the vector element is divergence free and the fields can be continuous and discontinuous where applicable [36]. The essential boundary conditions for the second order partial differential wave equations are the Dirichlet and Neumann conditions which require the fields and their first derivatives to match at the boundaries.

Even with the above approach, the extremely large number of equations to be solved and solutions to be organized needs to be handled in a precise manner. Also, the desire for a relatively fast solution to the model's equations is required. PARDISO enables fast solutions by pivoting and block diagonal pivoting of the matrices. Matrix pivoting involves the interchanging of rows and columns to more quickly solve the system. The pivot in a matrix is the element on the diagonal by which other elements are divided. In matrix pivoting, a preferred element is placed on the diagonal to grant a solving advantage [37]. 
The use of PARDISO limits regular pivoting when necessary, since this is not always the most effective method, by using block diagonal pivoting which obtains an equivalent system which is more diagonally-dominant in the usually sparse matrices of electromagnetic wave simulations. While preprocessing reduces partial pivoting, or matrix row interchange, and speeds-up the factorization, a solver must be robust enough to solve Maxwell's equations. Pivoting grants this robustness and so must be included in PARDISO. These block diagonal pivots are a compromise of the speed and robustness [38].

\subsection{Building Models}

The models in COMSOL are created using MATLAB scripts and a graphical user interface (GUI) which consists of a workspace where the geometry, variables, parameters, and physics are input and a computer aided drafting region in which the model is viewed. While the GUI provides a convenient platform for some model manipulation, MATLAB often allowed for better model controls, specifically parameterization, plotting, and troubleshooting.

The nanocone models are first declared to operate in the frequency domain and a 1-, 2, or 3-dimensional workspace is specified. Parameters are defined to be varied during computation and variables are declared. The parameters varied include the incident light angle, nanocone dimensions, the wavelength of light, and the index of refraction. The variables of note are the wave vector components which are needed to properly define the 
propagation in 2- and 3-dimensions. Angular dependence is built-in to allow for varied incident light to interact with the modeled structure. The geometry is then built using either preprogrammed primitives, free-drawn curves, or inputted values from the user. Geometric spacing of the mesh elements is vitally important to model functionality, as well as physical accuracy, since the mesh elements need to properly dissect the structure without distorting or inverting the elements. Once the geometry is built to specification, the material properties are defined and assigned to the various spatial regions. Material properties tensors are on-diagonal, identical elements to specify a linear, isotropic, and homogeneous medium. The materials can also be defined as an interpolative function with a predetermined dependence on a particular quantity. This allows for a wavelengthdependent index of refraction to vary with the parameterization of the incident light's wavelength. Both the real and imaginary parts of the refractive index can be defined, but only the real portion is considered in the models presented here.

Next the electrodynamics, including the boundary conditions of the system, are defined. The modeled wave equation acts over the entire global coordinate system and is of the form:

$$
\vec{\nabla} \times(\vec{\nabla} \times \vec{E})-k_{0}^{2} \epsilon_{r} \vec{E}=0
$$

The electric displacement field model is calculated from the material property equation:

$$
\epsilon_{r}=(n-i k)^{2}
$$

COMSOL users a minus sign for the imaginary part although it can be a positive sign by convention. The refractive index has a real part, $n$, of the relative permittivity and the 
imaginary portion, $k$, which is responsible for the absorption by the material. In the calculations presented here, only the real portion is considered. The absorption in the visible spectrum is minimal and so can be discounted, as shown in section 3.8.3. The boundary conditions are more detailed in scope and are discussed in the chapter section 3.6 .

\subsection{Meshing Requirements}

Once the boundary conditions are defined appropriately, which can be non-trivial, the mesh is created. A mesh is a sampling of the geometry in order to numerically compute a solution. The mesh elements need to be small enough to impose linearity on the system components, but large enough to enable computation by the computer. An element size of one-tenth of the wavelength $(\lambda)$ is desired to produce accurate models [39]. The meshing requirements put a considerable strain on the computer, by not only lengthening computation times, but causing a run-time failure due to insufficient memory. A simple halving of the mesh size will cause a minimum of an eight-fold increase in computational time due to a 3-dimensional geometry. To accurately simulate the required models, a 176 gigabyte server was constructed to allow for such small mesh elements. Typical meshes can range from 50,000 elements to 5,000,000 elements, depending on the complexity of the geometry, in order to allow for the required $\lambda / 10$ sizing. 


\subsection{Nanocone Model Geometry and Mesh}

The nanostructure models consist of a single unit cell which is made periodic in the $\mathrm{x}$ and y-directions. Modeling of the periodic geometry limits the capabilities of both the software and computer. The scale, physics, and post-processing are computationally intensive and need to have very well-defined geometry, mesh elements, and boundary conditions. The geometry consists of a single unit cell repeated to the computer's infinity limitations to simulate a small structured portion on a much larger substrate. A single nanocone unit cell is shown in figure 3.1. The dimensions for this model are $500 \mathrm{~nm}$ in height and 50 nanometers in radius to effectively demonstrate the geometry, even though most models can vary widely. The cone has material properties defined to match zinc oxide, the substrate on which the cone is placed has properties defined to match those of silicon, and the portion surrounding the nanocone is vacuum to approximately simulate air qualities. 

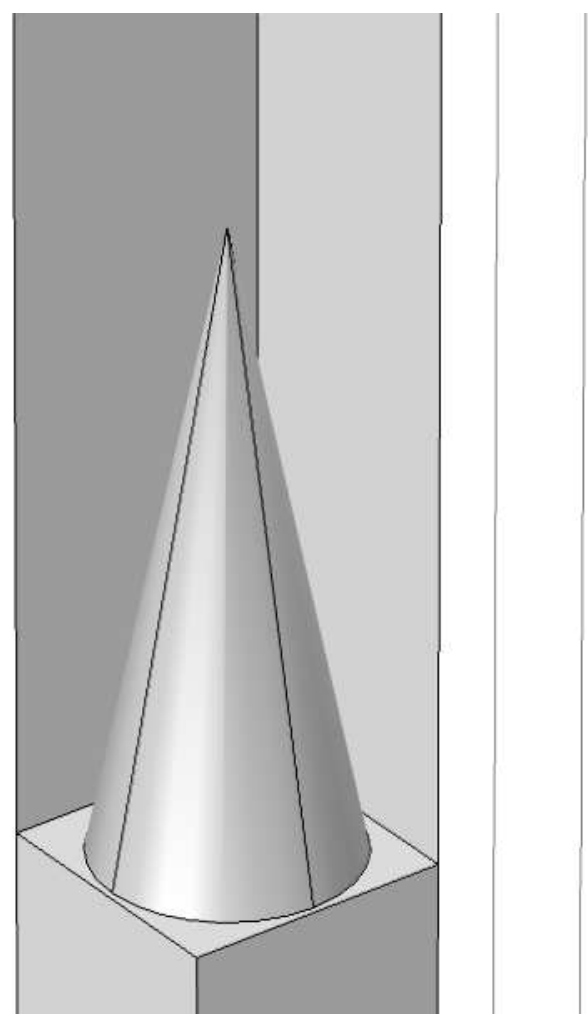

Figure 3.1. Single nanocone geometry.

The bulk of the volume is meshed with unstructured free tetrahedral elements to minimize the filling requirements and still properly represent the unit cell. However, the geometry is also periodic, contains small cornering portions, and has a central structure better sampled from triangular surface elements.

To ensure accurate replication of the unit cell's faces, the repeated boundary surfaces must be copied from the source boundary to the destination boundary. This ensures proper definition of the periodicity and wave vectors in adjacent virtual unit cells. The nanocone and substrate surfaces are also defined to have separate surface meshes to sufficiently fill the spatially-constricted geometry areas and to account for curved surfaces. This also eliminates inverted elements, which are volumetric inversions created 
from improper element definition and cause errors. Nanocone surface meshing elements are defined as triangular elements with a growth rate of 1.1-1.4. The growth rate dictates the size adjustment in adjacent locations and is kept close to unity in the areas with complex geometry to smoothen the transition to larger elements. The surface mesh maintains higher quality elements at the local nodal points by higher order differential functions which better represents the geometric surface variations with triangular-totetrahedral transition points. Controlling the resolution of curvature also controls the density in curvilinear regions. The typical conical model has a mesh curvature resolution programmed to 0.3 . A value of 0.3 allows the mesh to accurately recreate the geometry without needing exceedingly small elements. Such small elements would drastically increase the computational time and power needed. The mesh for the $500 \mathrm{~nm}$ high $\mathrm{x} 50$ $\mathrm{nm}$ radius cone is shown in figure 3.2 . 


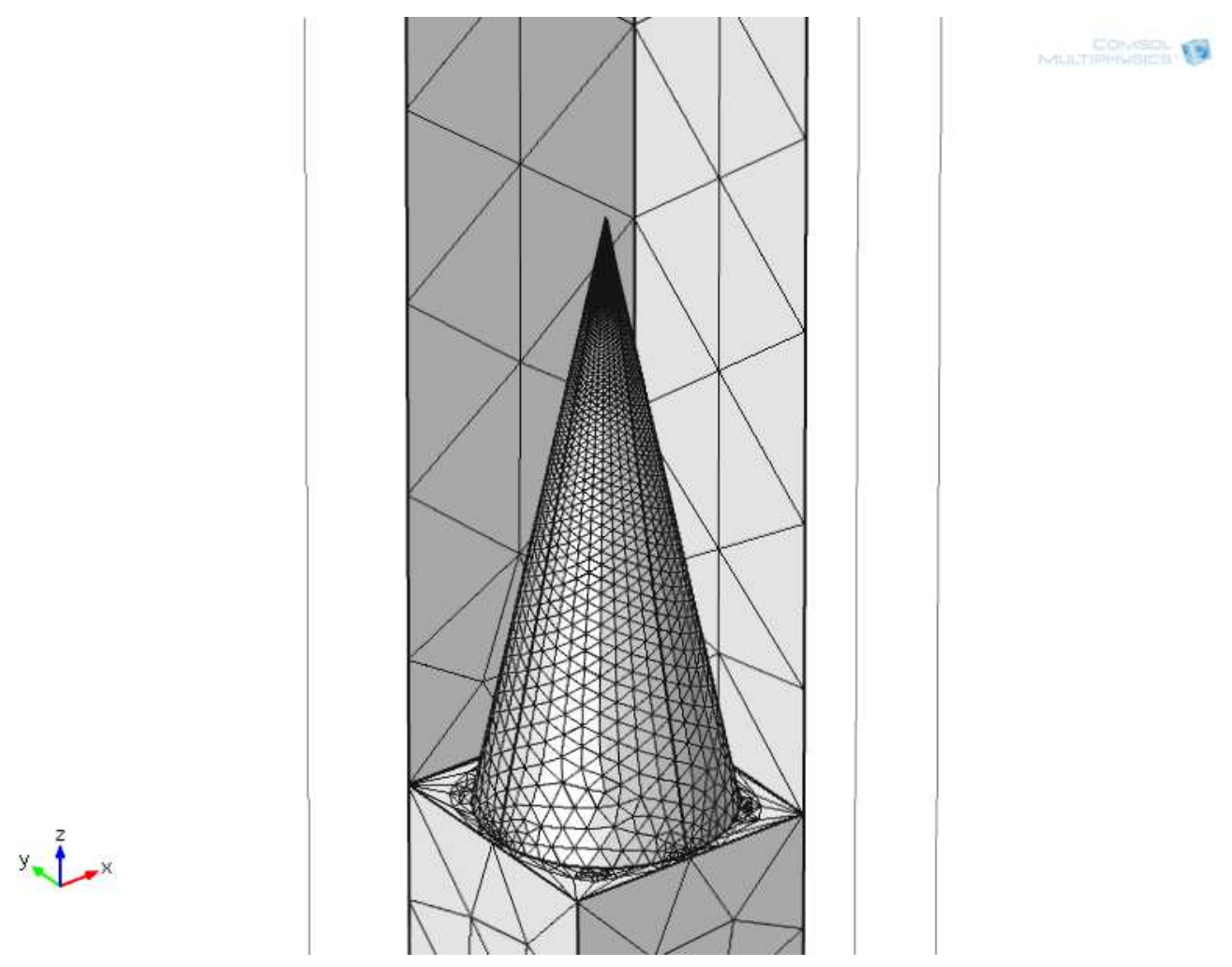

Figure 3.2. Nanocone mesh.

\subsection{Boundary Conditions}

Three types of boundary conditions are used in the nanostructure models including a) port boundaries which are transparent to plane waves and where the incoming waves are excited, b) continuity boundaries which satisfy the continuity of tangential fields, and c) periodic boundaries which allow for infinite periodicity of the model's defined unit cells. The usage of periodic boundary conditions allows the user to create an infinite array of nanostructures by periodically repeating the defined unit cell. This simulates a portion of a much larger area. Continuity boundary conditions are utilized when the tangential 
components of the field vectors are to be continuous. The boundary conditions are shown in figure 3.3.

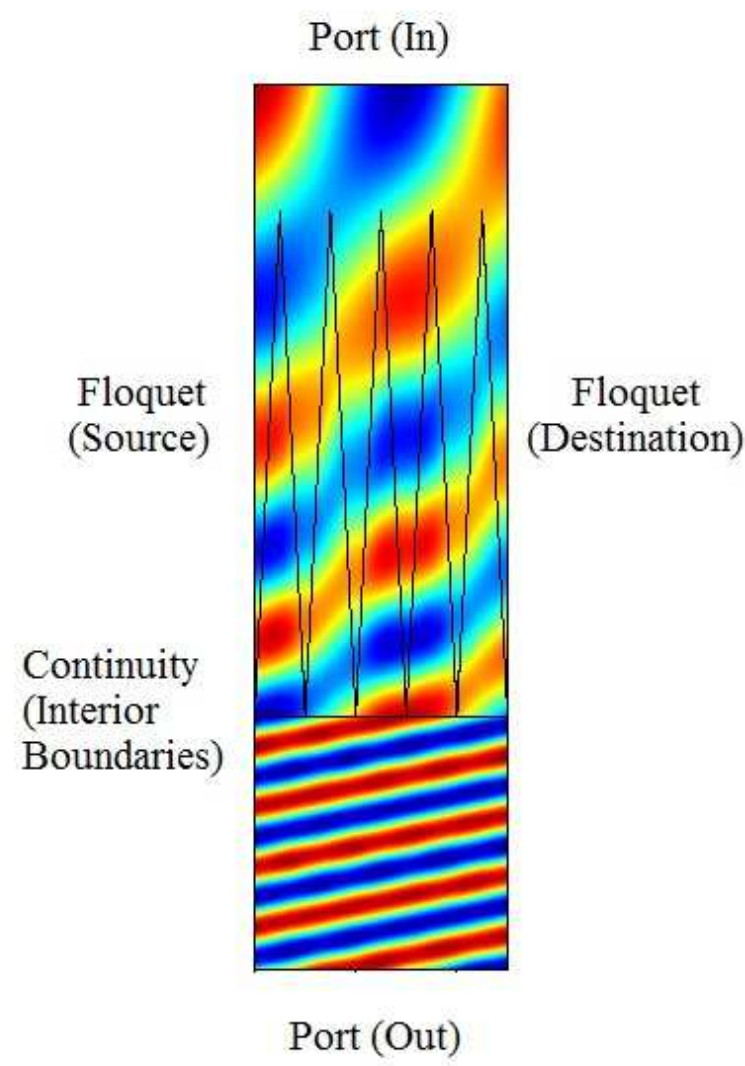

Figure 3.3. Boundary conditions for a 2-dimensional, periodic nanoridge cell.

\subsubsection{Continuity Boundary Conditions}

For an arbitrary surface, an infinitesimal surface $S$ can be defined to compute the surface integral. The surface integral can be converted to a contour integral, with closed path $C$ along the differential length $\overrightarrow{d l}$, according to Stokes' Theorem. As the surface $S \rightarrow 0$, the leftmost and rightmost segments of the path drop and only the top and bottom portions are left. This integral becomes a simple subtraction of the fields in the respective regions 
upon evaluation. The fields $\vec{E}_{1}$ and $\vec{E}_{2}$ along the differential lengths $d l$ of region 1 and 2 , which are equal, are just the tangential components of the fields $E_{1 t}$ and $E_{2 t}$ and are perpendicular to each differential length. The tangential components of the fields can then be shown to be continuous. This result holds for the electric and displacement vectors [40].

$$
\int_{S} \vec{\nabla} \times \vec{E} \cdot \hat{n} d A=\oint_{C} \vec{E} \cdot \overrightarrow{d l}=0
$$

As the surface $S \rightarrow 0$

$$
\begin{gathered}
\left(\vec{E}_{2} \cdot \overrightarrow{\Delta l}-\vec{E}_{1} \cdot \overrightarrow{\Delta l}\right)=0 \\
\left(E_{2 t}-E_{1 t}\right) \Delta l=0 \\
E_{1 t}=E_{2 t}
\end{gathered}
$$

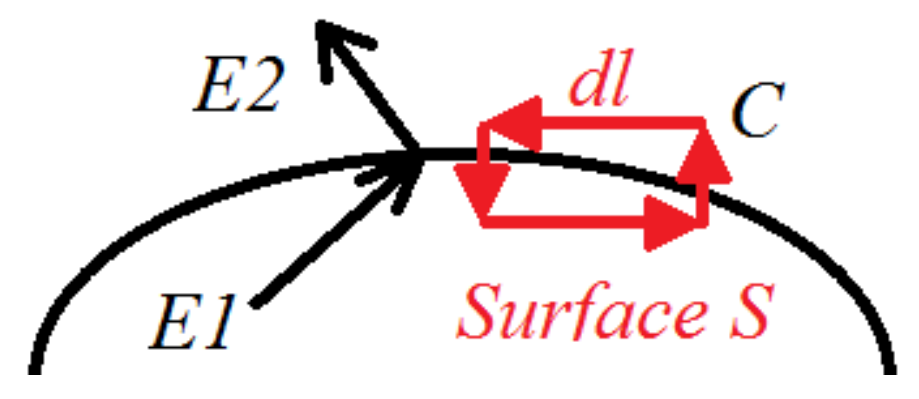

Figure 3.4. An arbitrary surface splitting two regions containing electric fields $\vec{E}_{1}$ and $\vec{E}_{2}$ respectively, with infinitesimally small length $\overrightarrow{d l}$, closed path $\mathrm{C}$, and integration surface $S$. 
The $\vec{H}$ field boundary conditions are formulated the same way. For an arbitrary boundary, the tangential components must be continuous. Applying the boundary condition to Maxwell's equation [40]:

$$
\int_{S} \vec{\nabla} \times \vec{H} \cdot \hat{n} d A=\oint_{C} \vec{H} \cdot \overrightarrow{d l}=\oint_{C}\left(\overrightarrow{J_{f}}+\frac{\partial \vec{D}}{\partial t}\right) \cdot \overrightarrow{d l}
$$

With no surface currents:

$$
\begin{gathered}
\left(H_{2 t}-H_{1 t}\right) \Delta l=\frac{\partial}{\partial t}\left(\left(D_{2 t}-D_{1 t}\right) \Delta l\right)=0 \\
H_{1 t}=H_{2 t}
\end{gathered}
$$

\subsubsection{Periodic Boundary Conditions}

The periodic boundaries at the right and left edges of the unit cell have Floquet periodicity applied. This allows for a translation of the unit cell to be studied, which is repeated to infinity, and creates infinitely many parallel unit cells extending in all directions to which the periodic condition applies. Periodic boundaries allow the simulation of very large systems, which otherwise might be impossible to model, by using a much smaller portion to populate the system through replication.

Floquet periodicity, sometimes called Floquet-Bloch periodicity, can only represent a periodic structure which is well-defined, such as the unit cell presented here. The incoming "source" field vector translates to the outgoing "destination" by a phase shift. 
In this way, the source is mapped onto the destination to repeat the structure over the period. COMSOL defines the periodic boundary phase shift as:

$$
\varphi=\vec{k}_{F} \cdot\left(\vec{r}_{\text {dest }}-\vec{r}_{\text {src }}\right)
$$

This is simply the exponential term of a Bloch wave with $\vec{k}_{F}$ being the wave vector and $\left(\vec{r}_{\text {dest }}-\vec{r}_{s r c}\right)$ being the distance between the wave coming into, and going out of, an arbitrary cell of the system. These boundaries are defined on the unit cell's vertical boundaries, but can be any cell with the proper definition of the source and destination position vectors. With this phase shift, the Bloch waves at the boundary where the wave leaves the arbitrary cell take the form:

$$
\begin{aligned}
& \psi(\vec{r}+\vec{R})=\psi(\vec{r}) e^{-i(\vec{k} \cdot(\vec{r}+\vec{R}))}=\psi(\vec{r}) e^{-i(\vec{k} \cdot \vec{r})} e^{-i(\vec{k} \cdot \vec{R})} \\
& =\psi(\vec{r}) e^{-i\left(\vec{k} \cdot \vec{r}_{s c}\right)} e^{-i\left(\vec{k} \cdot\left(\vec{r}_{\text {dest }}-\vec{r}_{\text {src }}\right)\right)}=\psi(\vec{r}) e^{-i\left(\vec{k} \cdot \vec{r}_{\text {dest }}\right)}
\end{aligned}
$$

The symbol $\psi$ is representative of the electromagnetic fields and their associated wave behavior. The periodic condition for the Bloch waves in the model is satisfied by defining:

$$
\psi(\vec{r})=\psi(\vec{r}+\vec{R})=\psi(\vec{r}) e^{-i\left(\vec{k} \cdot \vec{r}_{\text {dest }}\right)}
$$

This allows the model to simulate an infinite array of unit cells with any geometry. 


\subsubsection{Port Boundary Conditions}

Ports are used to drive electromagnetic waves into the region of interest and for calculating various quantities depending on the modeler's needs. The port boundaries are transparent to the waves passing through them [39]. The incident fields are user defined and are time-harmonic which allows a stationary solution. The wavelength is varied, with the angular dependence stated explicitly in the exponential terms. The direction of propagation is in the negative z-direction or from the top boundary down through the cell.

$$
\begin{gathered}
\text { TM }\left\{\begin{array}{c}
\text { incoming: } H_{0}=H_{0 y} e^{-i\left(k_{x_{i}}\right)}=H_{0 y} e^{-i\left(n_{\text {air }} k_{0} \sin (\text { alpha })\right)} \\
\text { outgoing: } H_{0}=H_{0 y} e^{-i\left(k_{x_{t}}\right)}=H_{0 y} e^{-i\left(n_{S i} k_{0} \sin (\text { beta })\right)}
\end{array}\right. \\
T E\left\{\begin{array}{c}
\text { incoming: } E_{0}=E_{0 y} e^{-i\left(k_{x_{i}}\right)}=E_{0 y} e^{-i\left(n_{\text {air }} k_{0} \sin (\text { alpha })\right)} \\
\text { outgoing: } E_{0}=E_{0 y} e^{-i\left(k_{x_{t}}\right)}=E_{0 y} e^{-i\left(n_{S i} k_{0} \sin (\text { beta })\right)}
\end{array}\right. \\
\text { beta }=\sin ^{-1}\left(\frac{n_{\text {air }}}{n_{S i}} \sin (\text { alpha })\right)
\end{gathered}
$$

Where $k_{x_{i}}$ is the $\mathrm{x}-$ component of the incident wave vector and $k_{x_{t}}$ is the $\mathrm{x}-$ component of the transmitted wave vector.

In addition, the propagation constant is the absolute value of the wave vector normal to the top and bottom boundaries:

$$
\beta=\left\{\begin{array}{c}
\text { incoming: }\left|k_{z_{i}}\right|=\mid n_{\text {air }} k_{0} \cos (\text { alpha }) \mid \\
\text { outgoing: }\left|k_{z_{t}}\right|=\mid n_{S i} k_{0} \cos (\text { beta }) \mid
\end{array}\right.
$$




\subsection{Solution Formulation}

The port boundary conditions are utilized for computing the reflectance from the scattering parameter matrix, or S-parameters, by integration of the fields at the top surface. S-parameters can be associated with voltage reflection and transmission but they are defined in terms of the electric fields in high-frequency calculations. The S-parameter matrix for $\mathrm{n}$ ports is defined as:

$$
\boldsymbol{S}=\left[\begin{array}{ccc}
S_{11} & \cdots & S_{1 n} \\
\vdots & \ddots & \vdots \\
S_{n 1} & \cdots & S_{n n}
\end{array}\right]
$$

For the total electric field $\vec{E}_{\text {total }}$ representing the incident wave added to the reflected portion, an S-matrix element is calculated from the fields using [39]:

$$
S_{n n}=\frac{\int_{\text {port } n}\left(\left(\vec{E}_{\text {total }}-\vec{E}_{n}\right) \cdot \vec{E}_{n}^{*}\right) \cdot \hat{n} d A_{n}}{\int_{\text {port } n}\left(\vec{E}_{n} \cdot \vec{E}_{n}^{*}\right) \cdot \hat{n} d A_{n}}
$$

In terms of power flow, the S-matrix elements are formulated as follows:

$$
S_{n n}=\frac{\sqrt{\text { Power Reflected from Port } n}}{\sqrt{\text { Power Incident on Port } n}}=\frac{\sqrt{\hat{n} \cdot\left\langle\vec{S}_{1}\right\rangle}}{\sqrt{\hat{n} \cdot\left\langle\vec{S}_{0}\right\rangle}}=\frac{\sqrt{\hat{n} \cdot\left\langle\frac{1}{2} \operatorname{Re}\left(\vec{E} \times \vec{H}^{*}\right)_{1}\right\rangle}}{\sqrt{\hat{n} \cdot\left\langle\frac{1}{2} \operatorname{Re}\left(\vec{E} \times \vec{H}^{*}\right)_{0}\right\rangle}}
$$

Here, $\langle\vec{S}\rangle$ is the time-averaged Poynting vector which is allowed under a steady state condition for the incident and reflected power and only the real portion of the crossproduct is needed [40]. 
Transverse electric waves have a time-averaged Poynting vector projected onto the axis of propagation, in terms of $\vec{E}$ only, by replacing the magnetic field with the use of the triple cross-product substitution [39]:

$$
\begin{aligned}
& S_{n n_{T E}}=\left(\frac{\sqrt{\hat{n} \cdot\left\langle\frac{1}{2} \operatorname{Re}\left(\vec{E} \times \vec{H}^{*}\right)_{1}\right\rangle}}{\sqrt{\hat{n} \cdot\left\langle\frac{1}{2} \operatorname{Re}\left(\vec{E} \times \vec{H}^{*}\right)_{0}\right\rangle}}\right)_{T E}=\left(\frac{\sqrt{-\left\langle\frac{1}{2} \operatorname{Re}\left(\vec{E} \cdot\left(\hat{n} \times \vec{H}^{*}\right)\right)_{1}\right\rangle}}{\sqrt{-\left\langle\frac{1}{2} \operatorname{Re}\left(\vec{E} \cdot\left(\hat{n} \times \vec{H}^{*}\right)\right)_{0}\right\rangle}}\right)_{T E} \\
& =\left(\frac{\sqrt{-\left\langle\frac{1}{2} \operatorname{Re}\left(\vec{E} \cdot\left(-\frac{\beta_{1}}{\mu_{1} \omega_{1}}\right)_{1}\right\rangle\right.}}{\sqrt{-\left\langle\frac{1}{2} \operatorname{Re}\left(\vec{E} \cdot\left(-\frac{\beta_{0}}{\mu_{0} \omega_{0}}\right)\right)_{0}\right\rangle}}\right)_{T E}=\left(\sqrt{\frac{\left\langle\frac{\beta_{1}}{\mu_{1} \omega_{1}}\left|\vec{E}_{1}\right|^{2}\right\rangle}{\left\langle\frac{\beta_{0}}{\mu_{0} \omega_{0}}\left|\vec{E}_{0}\right|^{2}\right\rangle}}\right)_{T E}=\left(\sqrt{\frac{\frac{1}{Z_{T E}}\left|\vec{E}_{1}\right|^{2}}{\frac{1}{Z_{T E_{0}}}\left|\vec{E}_{0}\right|^{2}}}\right)_{T E}
\end{aligned}
$$

The permeability is equal to one in both media for all models used and the wave impedance for transverse electric propagation is defined as:

$$
Z_{T E}=\frac{\mu \omega}{\beta}
$$

In this way, the reflectance is calculated as the square of the first S-parameter matrix element which would be incident through the initial port and reflected back through the same port. This becomes a ratio of the square of the field magnitudes:

$$
S_{11_{T E}}^{2}=R_{T E}=\left(\frac{\frac{1}{Z_{T E_{1}}\left|\vec{E}_{1}\right|^{2}}}{\frac{1}{Z_{T E_{0}}}\left|\vec{E}_{0}\right|^{2}}\right)_{T E}=\left(\left\langle\frac{\left|\vec{E}_{1}\right|^{2}}{\left|\vec{E}_{0}\right|^{2}}\right)_{T E}\right.
$$

For transverse magnetic waves: 


$$
\begin{aligned}
& S_{n n_{T M}}=\left(\frac{\sqrt{\hat{n} \cdot\left\langle\frac{1}{2} \operatorname{Re}\left(\vec{E} \times \vec{H}^{*}\right)_{1}\right\rangle}}{\sqrt{\hat{n} \cdot\left\langle\frac{1}{2} \operatorname{Re}\left(\vec{E} \times \vec{H}^{*}\right)_{0}\right\rangle}}\right)_{T M}=\left(\frac{\sqrt{\left\langle\vec{H}^{*} \cdot \frac{1}{2} \operatorname{Re}(\hat{n} \times \vec{E})_{1}\right\rangle}}{\sqrt{\left\langle\vec{H}^{*} \cdot \frac{1}{2} \operatorname{Re}(\hat{n} \times \vec{E})_{0}\right\rangle}}\right)_{T M} \\
& =\left(\frac{\sqrt{\left\langle\vec{H}^{*} \cdot \frac{1}{2} \operatorname{Re}\left(\frac{\epsilon_{1} \omega_{1}}{\beta_{1}} \vec{H}\right)_{1}\right\rangle}}{\sqrt{\left\langle\vec{H}^{*} \cdot \frac{1}{2} \operatorname{Re}\left(\frac{\epsilon_{0} \omega_{0}}{\beta_{0}} \vec{H}\right)_{0}\right\rangle}}=\left(\frac{\sqrt{\left\langle\vec{H}^{*} \cdot \operatorname{Re}\left(\frac{1}{Z_{T M_{1}}} \vec{H}\right)_{1}\right\rangle}}{\sqrt{\left\langle\vec{H}^{*} \cdot \operatorname{Re}\left(\frac{1}{Z_{T M_{0}}} \vec{H}\right)_{0}\right\rangle}}\right)_{T M}\right. \\
& =\left(\sqrt{\left.\frac{\frac{1}{Z_{T M_{1}}\left|\vec{H}_{1}\right|^{2}}}{\frac{1}{Z_{T M_{0}}}\left|\vec{H}_{0}\right|^{2}}\right)_{T M}}\right.
\end{aligned}
$$

The impedance for transverse magnetic propagation is defined as:

$$
Z_{T M}=\frac{\beta}{\epsilon \omega}
$$

The reflectance becomes:

$$
\left.S_{11_{T M}}^{2}=R_{T M}=\left(\frac{\frac{1}{Z_{T M_{1}}}\left|\vec{H}_{1}\right|^{2}}{\frac{1}{Z_{T M_{0}}}\left|\vec{H}_{0}\right|^{2}}\right\rangle\right)_{T M}=\left(\left\langle\frac{\left|\vec{H}_{1}\right|^{2}}{\left|\vec{H}_{0}\right|^{2}}\right\rangle\right)_{T M}
$$

\subsection{Model Verification}

The formulation of a multifaceted model requires multiple checks on its validity to verify the computational integrity of the software and solution viability of the models. A check 
on the proper domain scaling is done for periodic boundary conditions. Also, the known Fresnel equations are modeled to establish a baseline reflection model. The ability to neglect the absorption is tested and the nanocone model is varied to reproduce the familiar Fresnel solutions.

\subsubsection{Periodic Boundary Conditions from Single Cell Grouping}

The Floquet-Bloch boundary conditions must repeat the simple unit cell appropriately when applied to a model. This is verified by computing the fields for non-periodic cells and aligning them at the side boundaries to show an effective repetition of the central cell. As the number of non-periodic unit cells are increased, the appearance of periodicity becomes evident. Figures $3.5 \mathrm{a}-3.5 \mathrm{c}$ show the usage of single, non-periodic unit cells increasing in number from a single domain to multiple domains for a simple, 2dimensional geometry consisting of a single nanopillar. The five domain model is compared with the infinitely periodic model in figure 3.6. As the number of single domains placed side-by-side goes to infinity, the periodic condition is shown to exist demonstrating the proper usage of the Floquet-Bloch conditions in the models. 
a)

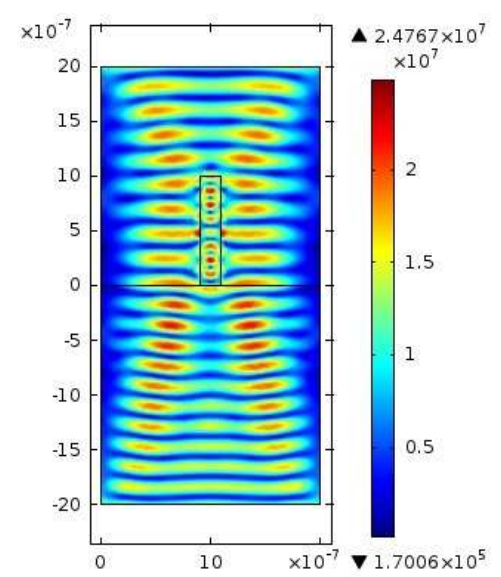

b)

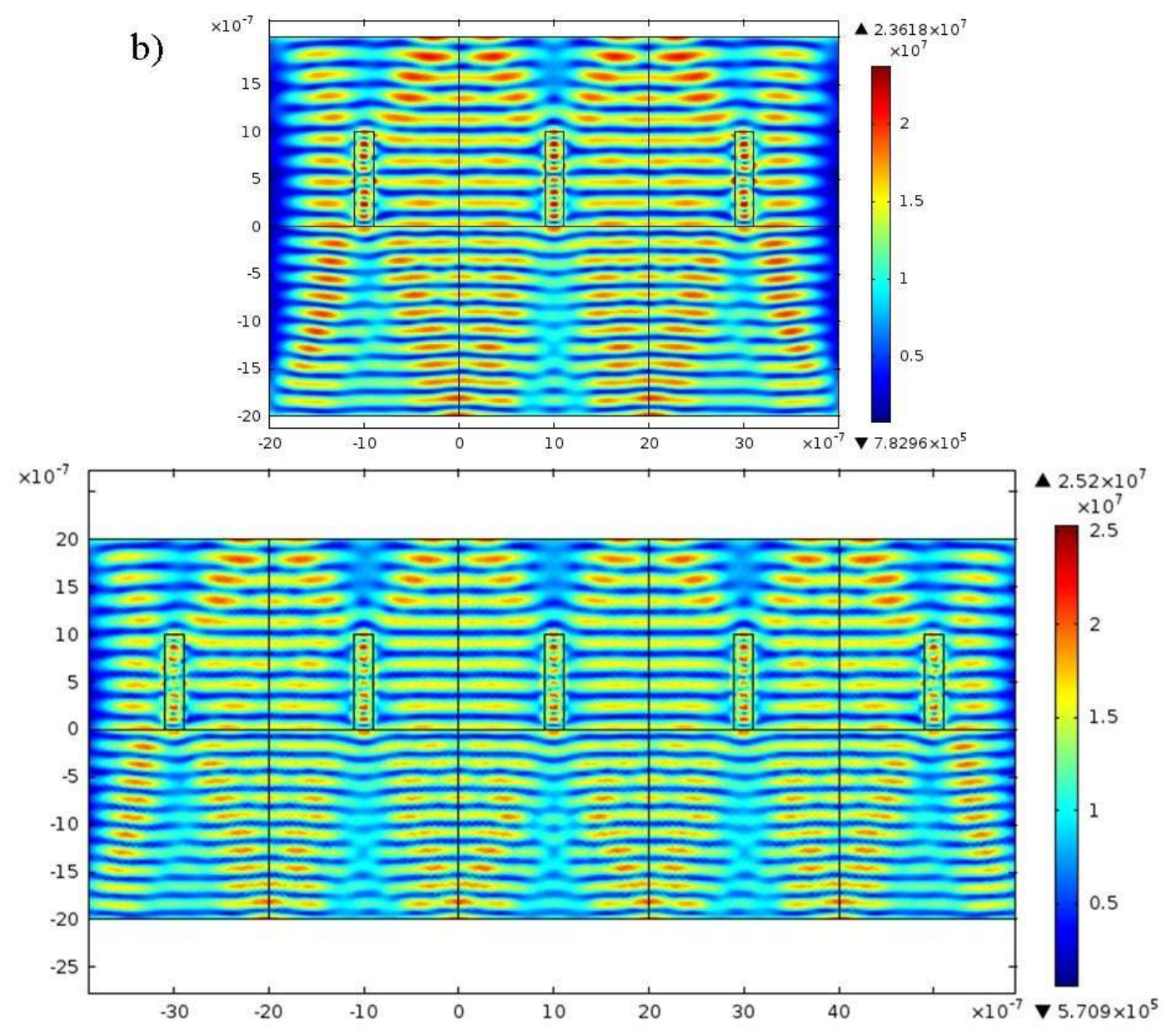

c)

Figure 3.5. Normalized electric field waveforms for the incoming and scattered fields with perpendicular incidence and TM polarization for non-periodic boundary conditions for a) single nanopillar domain, b) three nanopillar domains, and c) five nanopillar domains from a 2-dimensional model. 
1)

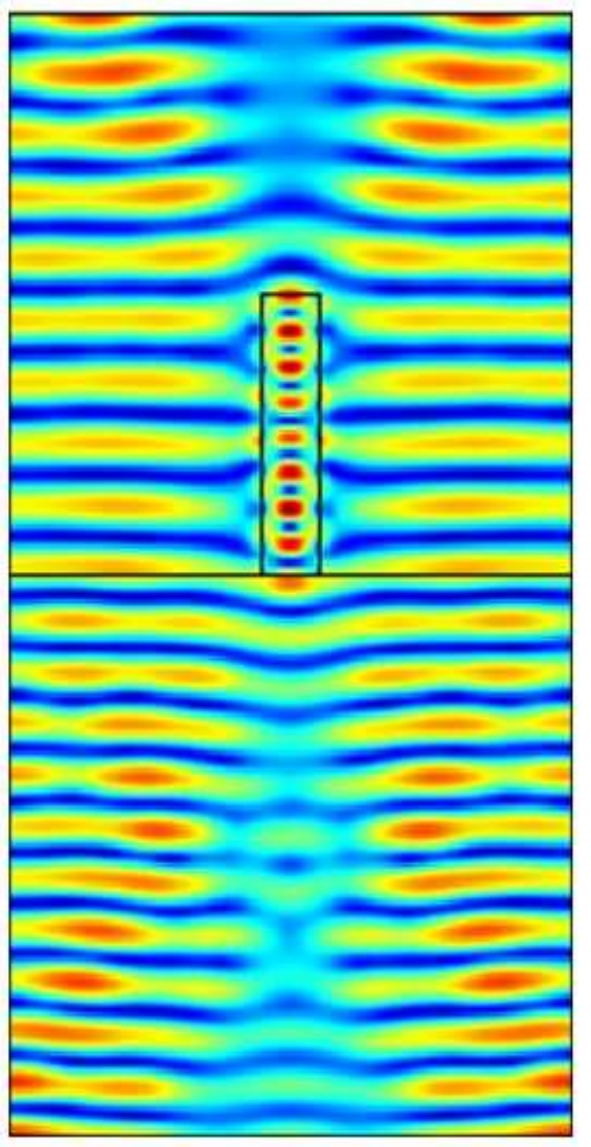

2)

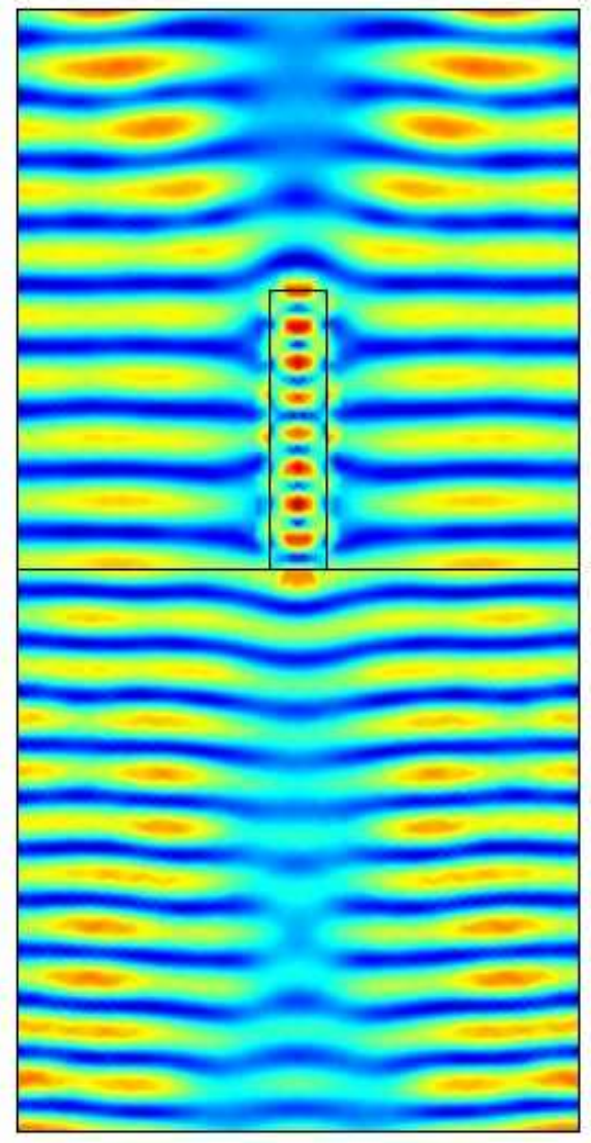

Figure 3.6. Comparison of 1) central unit cell of 5 domain model and 2) the unit cell from a periodic boundary model. As the number of single, non-periodic unit cells goes to infinity, the waveform repeats the periodic waveform.

\subsubsection{Fresnel Model Verification}

Confirmation of the computational effectiveness for reflection conditions with an air/glass interface between two infinite slabs in 3-dimensions is shown in figure 3.7. The model was constructed on the micron scale but very well could have been of any dimensions because of the infinite slab interface and depths. When compared with 
solutions calculated from the Fresnel equations, these models accurately recreate the Fresnel conditions. The analytical solutions are plotted for the TE and TM polarization equations:

$$
\begin{aligned}
& \text { TE Mode: } R=\left|\frac{n_{1} \cos \theta_{i}-n_{2} \cos \theta_{t}}{n_{1} \cos \theta_{i}+n_{2} \cos \theta_{t}}\right|^{2} \\
& \text { TM Mode: } R=\left|\frac{n_{1} \cos \theta_{t}-n_{2} \cos \theta_{i}}{n_{1} \cos \theta_{t}+n_{2} \cos \theta_{i}}\right|^{2}
\end{aligned}
$$

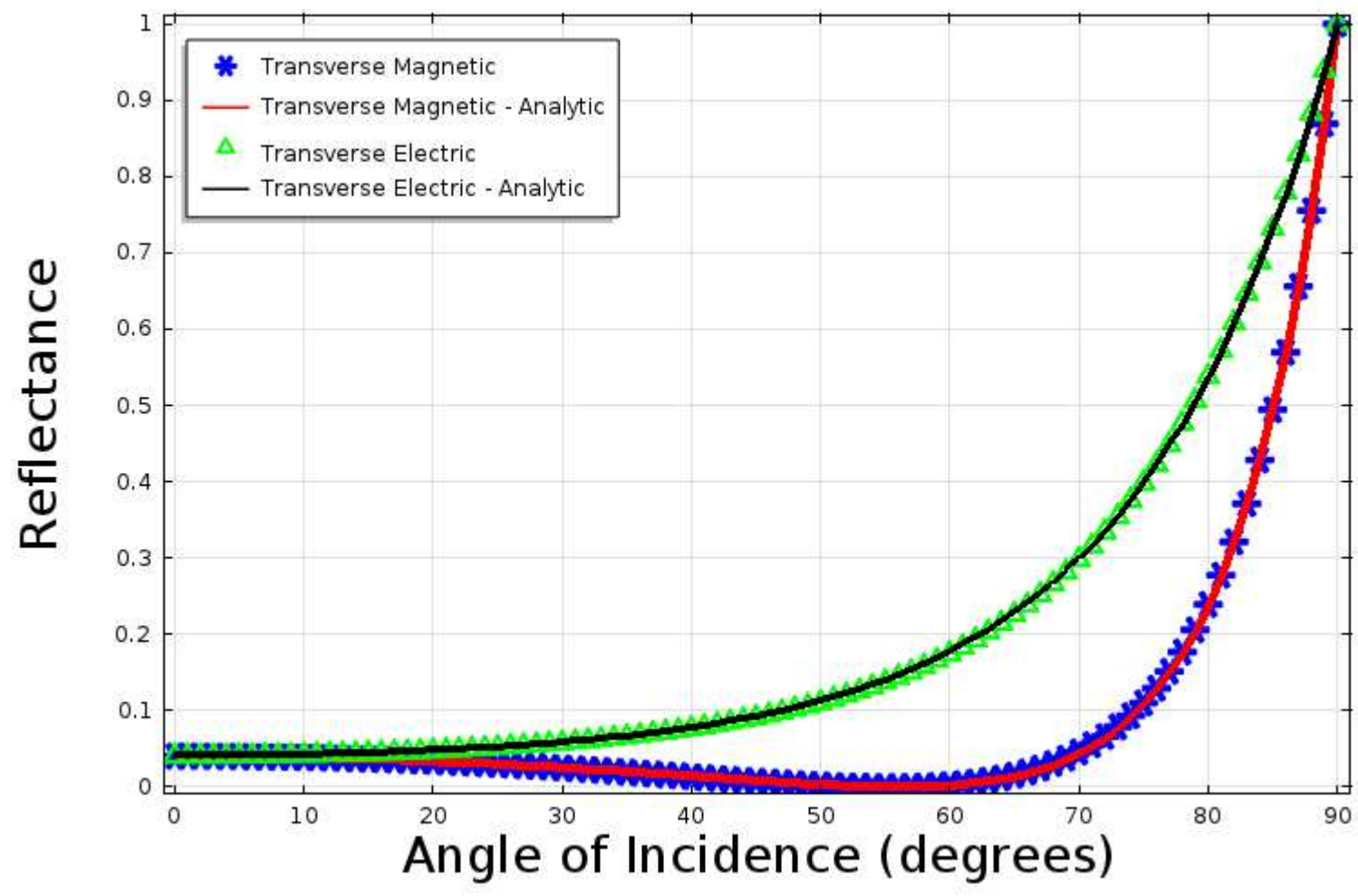

Figure 3.7. Fresnel model for $1 \mu \mathrm{m}$ high with a single interface between air and glass. The calculated analytical values and the simulation results are plotted. 


\subsubsection{Discounting Absorption}

To simplify the models, the absorption coefficient was set to zero. When silicon is modeled, a complex index of refraction is required to account for the absorptive properties in certain parts of the spectrum. In silicon solar cells, the thickness usually is chosen such that nearly all light is absorbed in the first passage. Therefore, we can neglect reflection and transmission at the back surface. Since reflection at the front surface and not transmission is considered, the need for better computational speed and functionality outweighs the need for a model including absorptive properties as long as the complex portion is minimal at the simulated wavelength, as proves to be the case.

Silicon has a complex dielectric function at $532 \mathrm{~nm}$ wavelength of [17]:

$$
\epsilon=(n-i k)^{2}=(4.1503-0.043933 i)^{2}
$$

The Fresnel equations for TM polarization is modeled for a single vacuum-silicon interface for both a complex dielectric function and a real one. The model has a varying angle of incident monochromatic light at a wavelength of $532 \mathrm{~nm}$. This wavelength is chosen since it is a standard type of green laser light used in laboratory settings.

While silicon has an absorptive element associated with its refractive index, an imaginary component causing losses in the medium, this is not taken into account in the subsequent models. The models are still well within the range of validity since, even with the dielectric losses included, the graphs are, for all practical purposes, identical (Figure 3.8). 


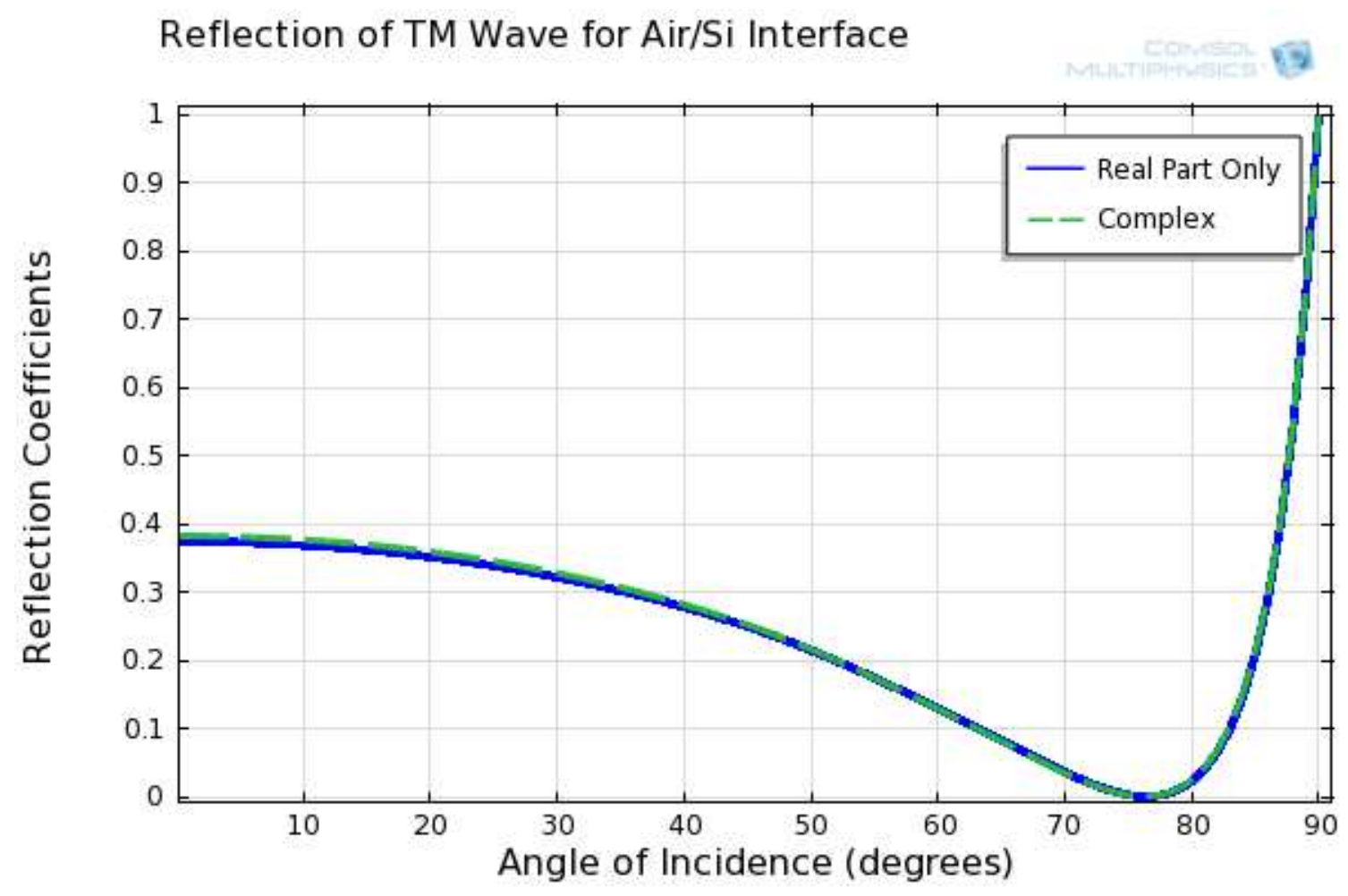

Figure 3.8. Comparison of real and complex dielectric functions for a single Air/Si interface with 532 nm incident light. 


\section{Results and Discussion}

Using the stated theory and computational methods, models were created to minimize the reflection of incident light on nanostructured $\mathrm{ZnO}$ on $\mathrm{Si}$ substrates for various conical widths, heights, and spatial orientations. The first models created were 2-dimensional to gain a working knowledge of the software, limit computational time and requirements, and to quickly adjust the various parameters. Three-dimensional models can then be constructed and simulated with more confidence in the computed solution.

\subsection{Nanocone Verification Model}

Verification of the nanocone model represents a challenge since the geometry is nonplanar. The problem was resolved by varying the structure to reproduce the Fresnel model by reducing the heights of the nanocones to zero by incremental steps and observing the reflection graph. Figure 4.1 shows the reduction of fixed, $50 \mathrm{~nm}$ radii $\mathrm{ZnO}$ nanocones from $250 \mathrm{~nm}$ height to planar, or $0 \mathrm{~nm}$ height on a $\mathrm{ZnO}$ substrate. Heights greater than this were used, but no significant difference was observed beyond $250 \mathrm{~nm}$ heights. These models were used to plot the reflectance graphs is Figure 4.2. The 3dimensional nanocones have index of refraction $n=2$, as does the substrate on which they are placed, and the top portion is air with an index $n=1$. As the nanocone height is reduced, the reflectance plots adjust to the accepted transverse magnetic (TM) plots for a planar interface. TM polarization is used for the incident light and the wavelength is 532 
$\mathrm{nm}$. The angle of incidence is increased from grazing to perpendicular incidence in steps of $3^{\circ}$. The plots converge to the Fresnel solution as the height is decreased to $0 \mathrm{~nm}$.
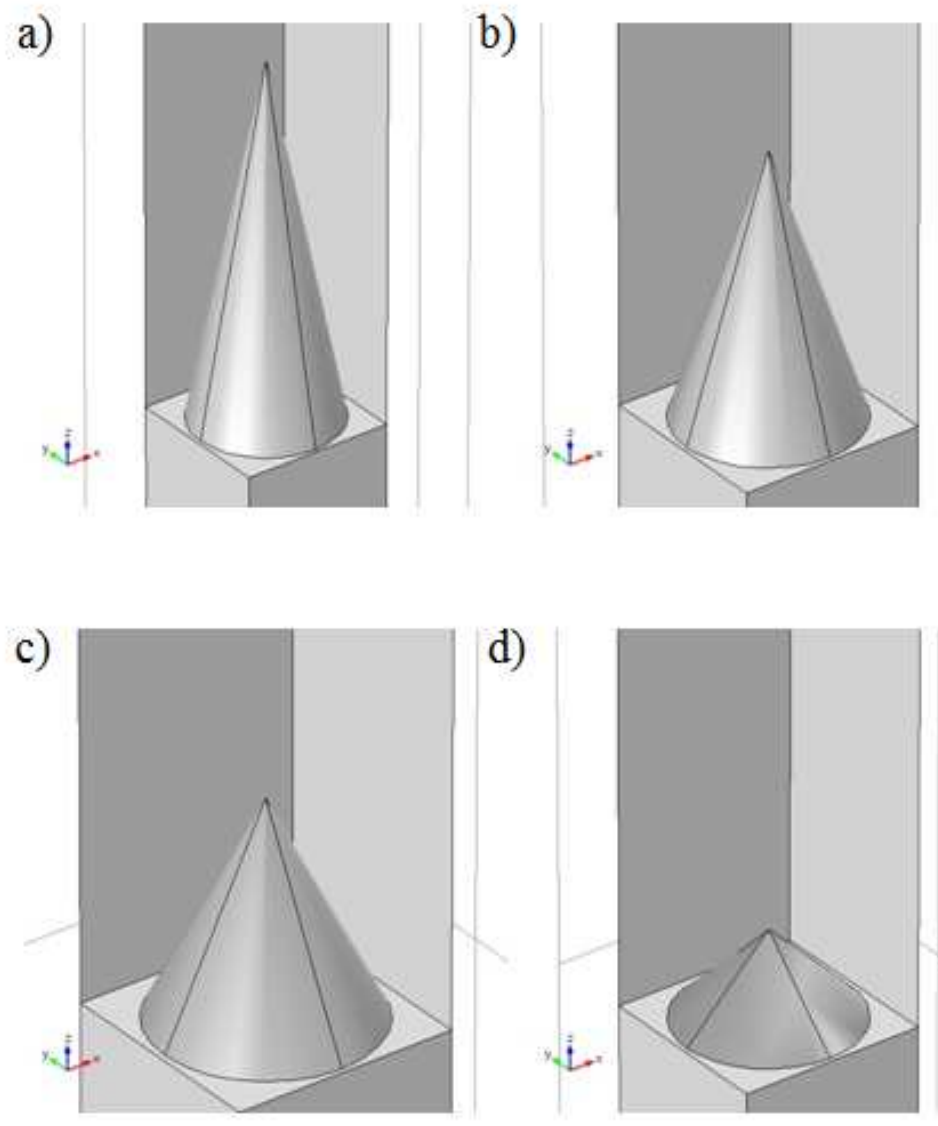

d)
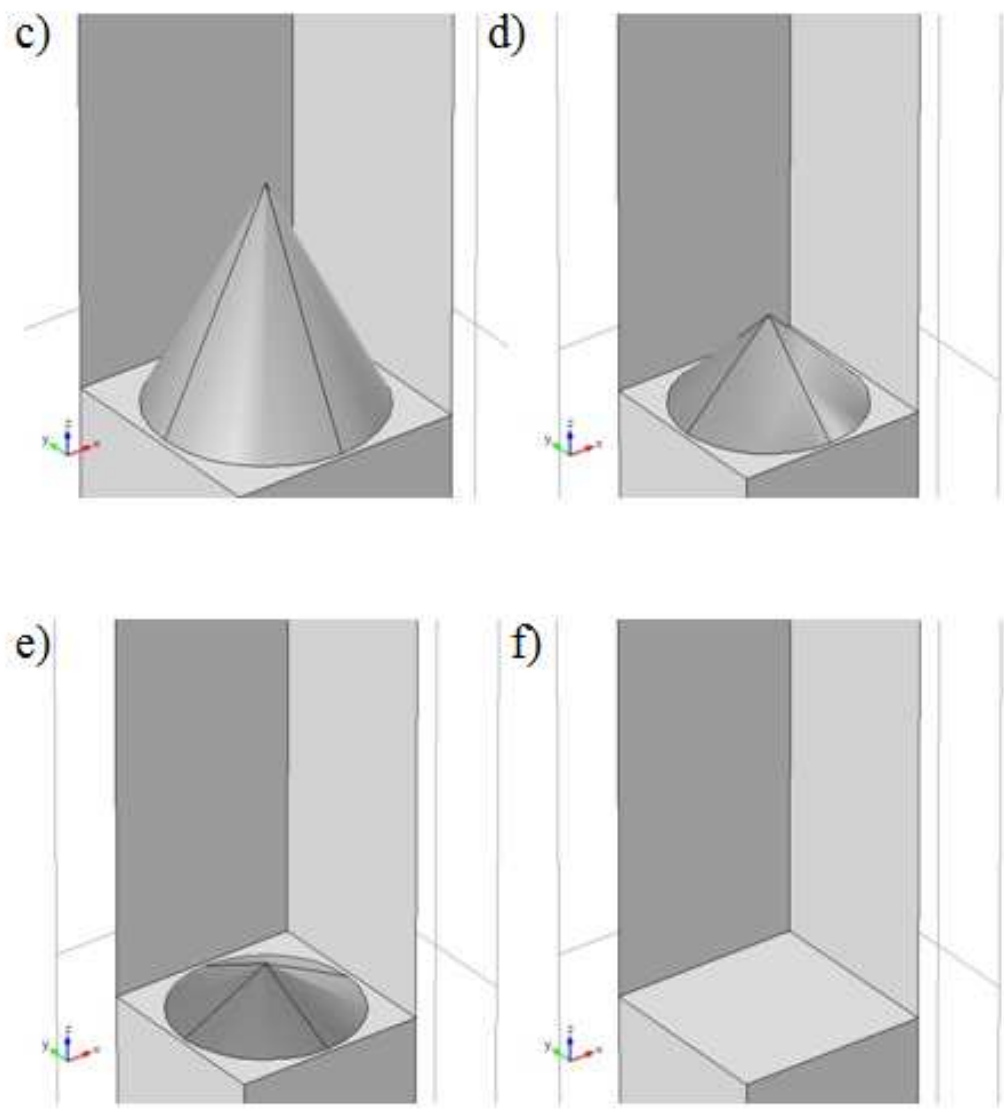

Figure 4.1. Height reduction of $\mathrm{ZnO}$ nanocones on $\mathrm{ZnO}$ substrate: a) $250 \mathrm{~nm}$ b) $150 \mathrm{~nm}$ c) $100 \mathrm{~nm}$ d) $50 \mathrm{~nm}$ e) $25 \mathrm{~nm}$ f) $0 \mathrm{~nm}$. Wavelength: $532 \mathrm{~nm}$, Refractive index: 2.03 . 

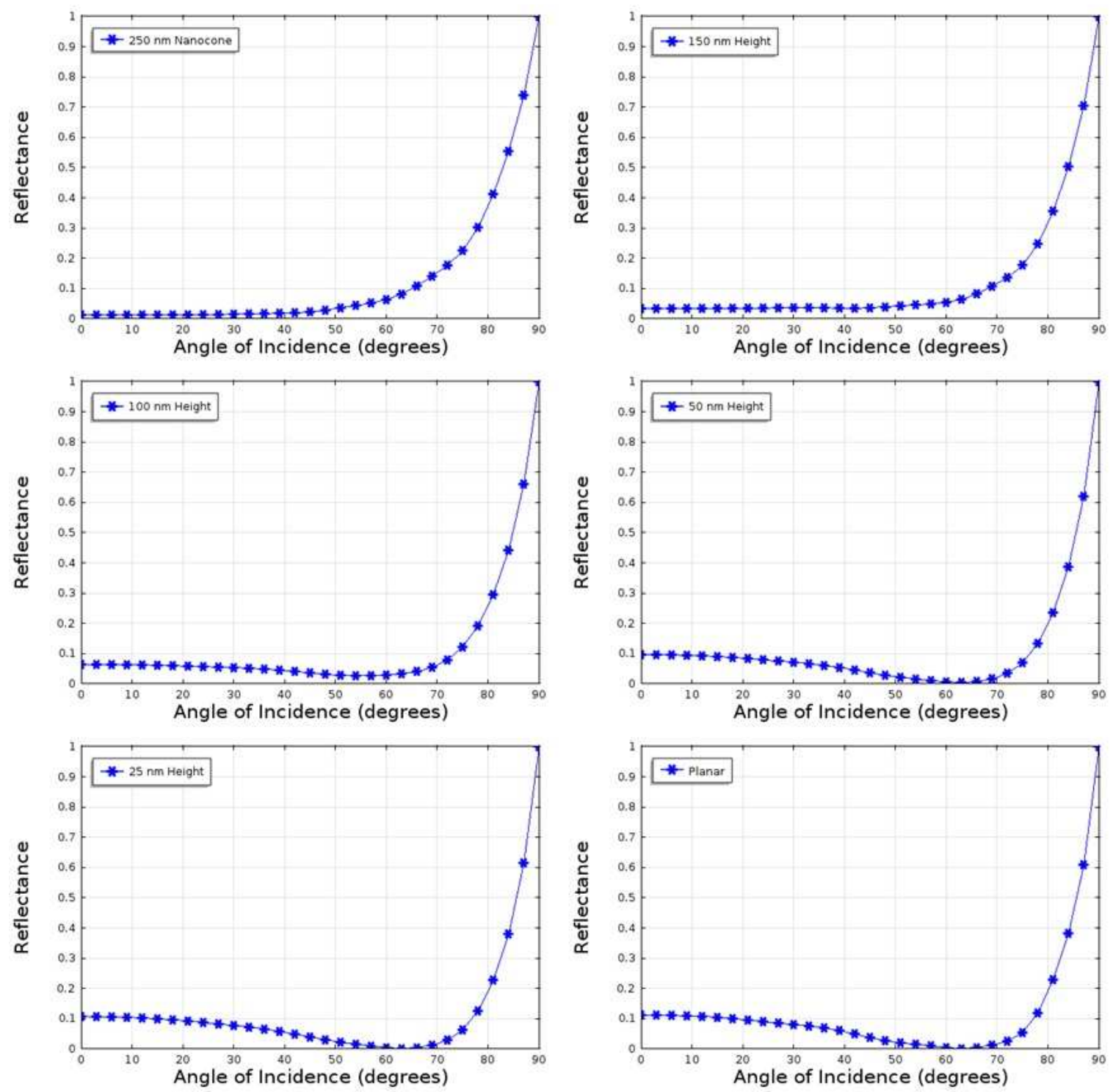

Figure 4.2. Reflectance plots for decreasing $\mathrm{ZnO}$ nanocone heights on $\mathrm{ZnO}$ substrate with TM polarization and a wavelength of $532 \mathrm{~nm}$ and refractive index of 2.03 . As the nanocone height goes to zero, the Fresnel solution is recreated, as seen in the bottom right.

\subsection{Two-Dimensional Models}

The 2-dimensional framework in COMSOL simulations is a cross-section of infinitely long structures. The structures in figure 4.3 are of nanoridges with heights of $1000 \mathrm{~nm}$ and base widths of $100 \mathrm{~nm}$. Magnetic (left) and electric (right) fields are plotted. Light 
incidence was defined at $60^{\circ}$ to the left from perpendicular. The nanoridge refractive index profile change acts to direct the light into the substrate, which is shown here as the rectangular base. This decreases the losses when compared to an air/substrate interface.

The reflectance plots show a decrease in total reflection versus a planar interface.
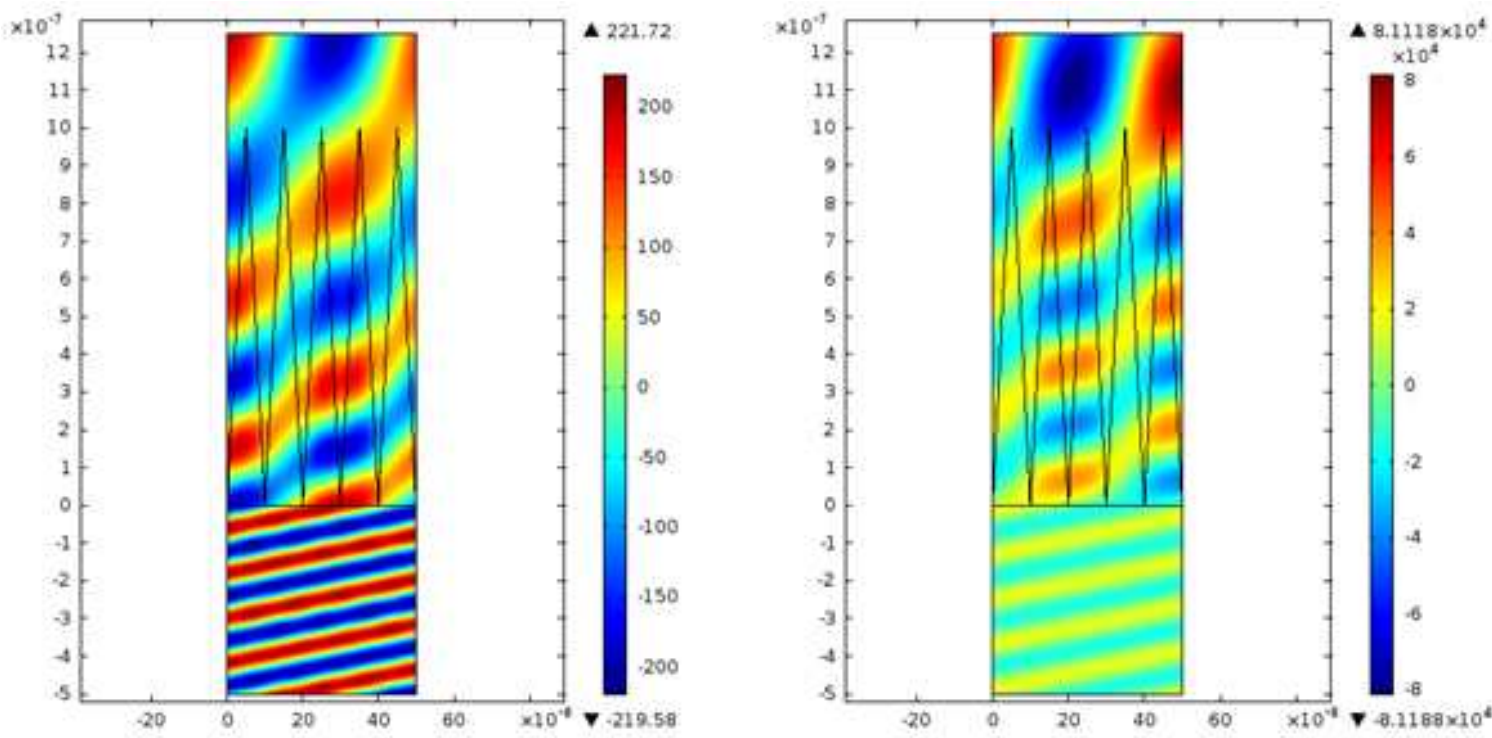

Figure 4.3. 2D $1000 \mathrm{~nm}$ height $x 100 \mathrm{~nm}$ width nanoridge plots of the magnetic field in TM propagation (left) and the electric field in TE propagation (right) for $60^{\circ}$ incidence. Wavelength: 500 nm, Refractive index: ZnO 2.0516, Si 4.29749.
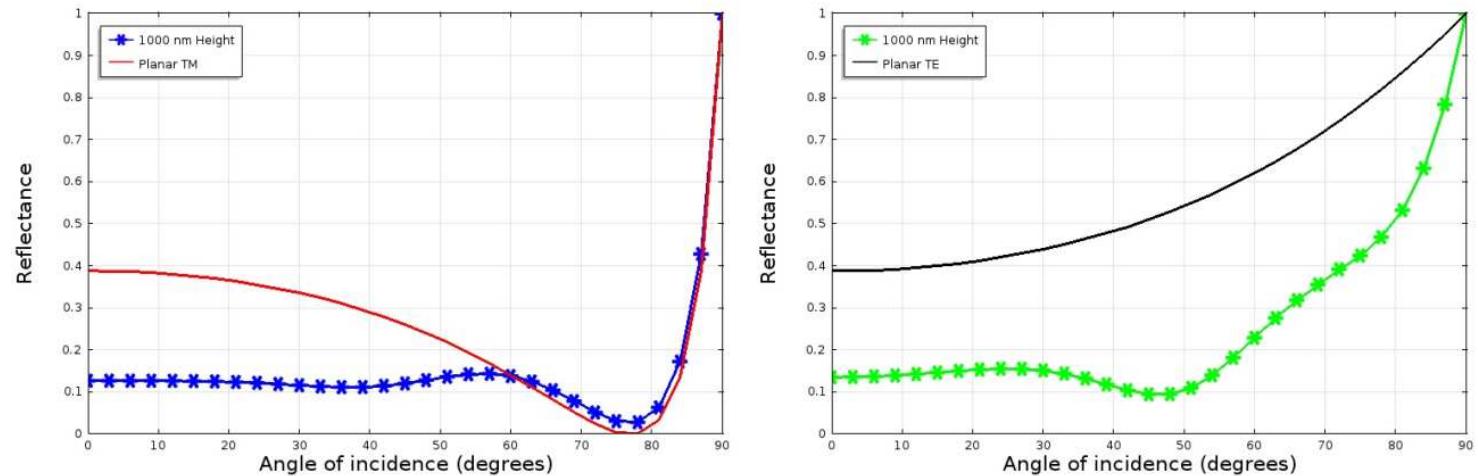

Figure 4.4. Reflectance vs. angle of incidence for $2 \mathrm{D} 1000 \mathrm{~nm}$ height $\mathrm{x} 100 \mathrm{~nm}$ width nanoridge structures for TM (left) and TE (right) polarizations. 


\subsection{Three-Dimensional Models}

\subsubsection{Single Nanocone with Periodic Boundaries}

Three-dimensional arrays were created with the use of a single $\mathrm{ZnO}$ nanostructure on an Si substrate and periodic boundaries in the $\mathrm{x}$-direction and $\mathrm{y}$-direction. The nanocone in figure 4.5 shows a single cone unit cell with $1000 \mathrm{~nm}$ height and $50 \mathrm{~nm}$ radius.

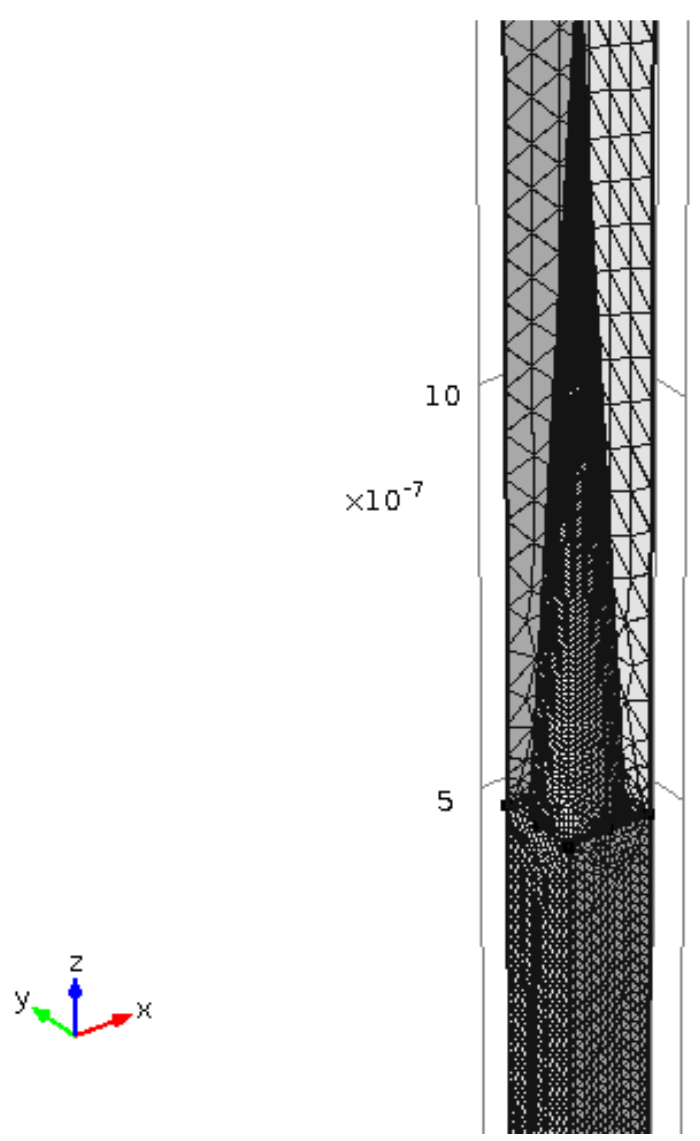

Figure 4.5. Single $1000 \mathrm{~nm}$ height $x 50 \mathrm{~nm}$ radius nanocone unit cell with periodic boundaries.

The reflectance curves for transverse magnetic and transverse electric polarizations for both the nanocone geometry and a planar interface are compared in figure 4.6. The total reflectance decreases with the addition of nanocone structures. The total reflectance for 
the nanocone models is about a factor of two lower than without the structuring for the TE case. The TM simulation shows approximately one-half the value of the planar model at perpendicular incidence to $5^{\circ}$ incidence, but the planar model continues toward a lower reflectance for increasing incident angle. The planar case crosses the nanocone case at $\sim 58^{\circ}$ as it the angle increases toward the Brewster angle. Beyond the $\sim 58^{\circ}$ angle, the planar TM case does not intersect the nanocone curve until $90^{\circ}$. The nanocone polarization dependence is much less pronounced than the planar case.

\section{Reflection vs. Incident Angle}

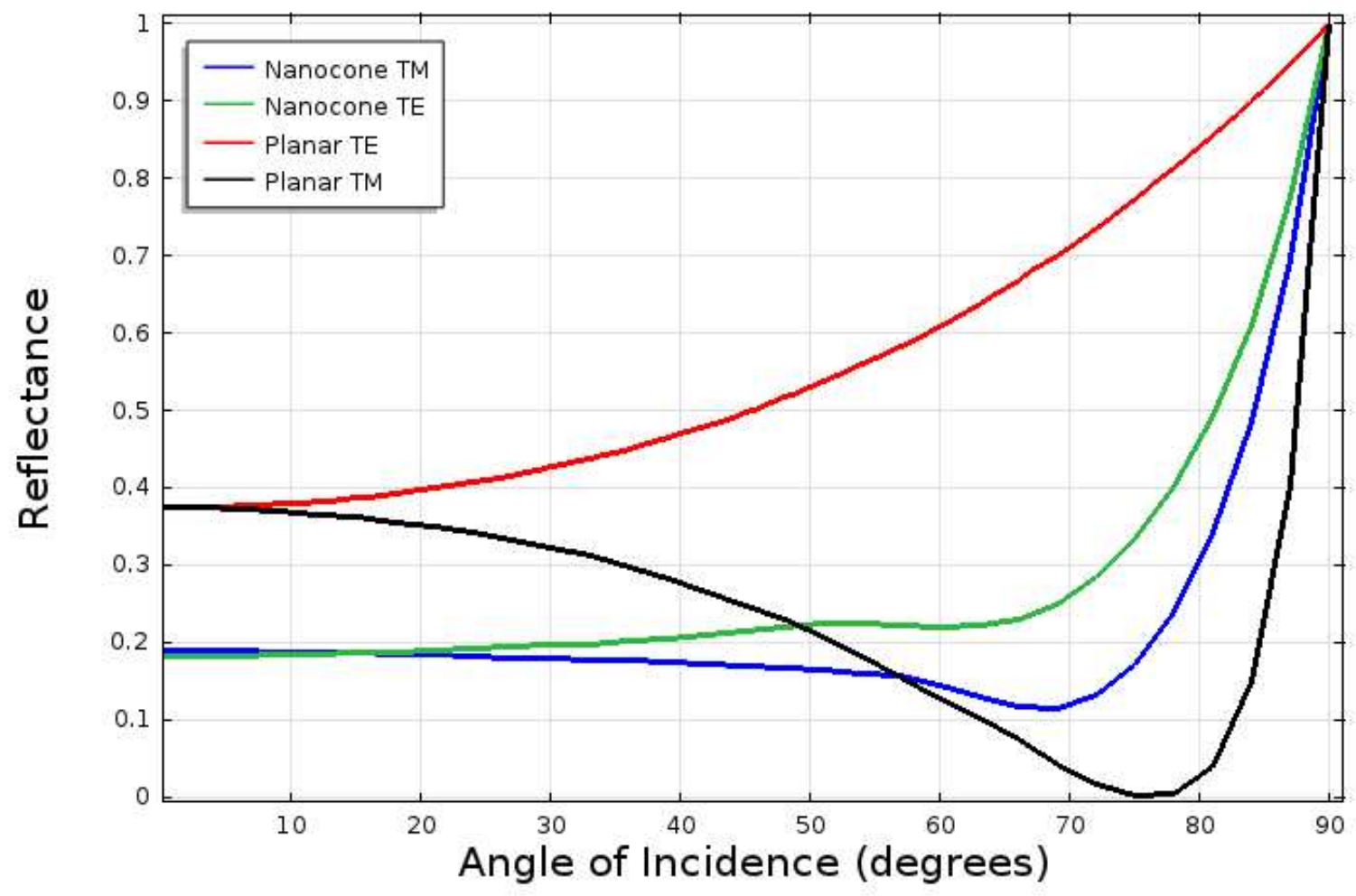

Figure 4.6. Reflectance vs. angle of incidence comparison for a $1000 \mathrm{~nm}$ height $x 50 \mathrm{~nm}$ radius nanocone model and a planar interface. Light wavelength is $500 \mathrm{~nm}$. 


\subsubsection{Height Variation}

The $\mathrm{ZnO}$ nanocone heights were varied to minimize the reflectance. Heights ranging from $200 \mathrm{~nm}$ to $1000 \mathrm{~nm}$ in increments of $200 \mathrm{~nm}$, as well as, 2 microns and 3 microns were modeled. In the TM case, the 400 and $800 \mathrm{~nm}$ heights showed the lowest reflectance. For the TE case, the shorter structure models showed an increase in reflectivity relative to the $1000 \mathrm{~nm}$ length, while the longer structures reduced reflection. Interesting features appear in the $800 \mathrm{~nm}$ graphs showing a Brewster angle-like dip for the higher incident angles. When simulations differing in wavelength were run, the 1000 $\mathrm{nm}$ nanocone model displayed the lowest reflectance. The incident light is $500 \mathrm{~nm}$ wavelength and the substrate is silicon.

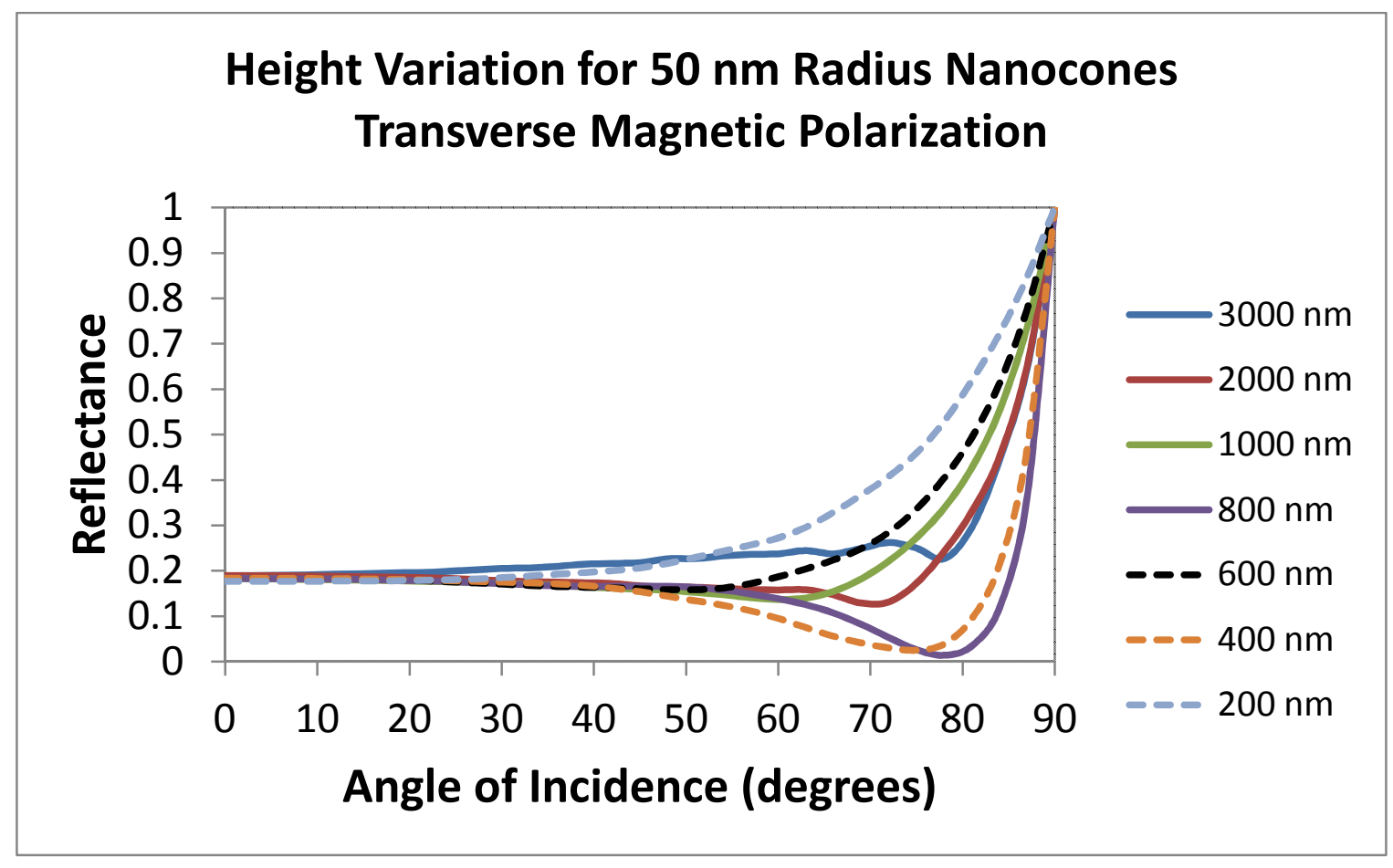

Figure 4.7. Reflectance vs. angle of incidence for varying nanocone heights with fixed radii of $50 \mathrm{~nm}$ for transverse magnetic fields. Wavelength: $500 \mathrm{~nm}$, Refractive index: Zno 2.0516, Si 4.29749. 


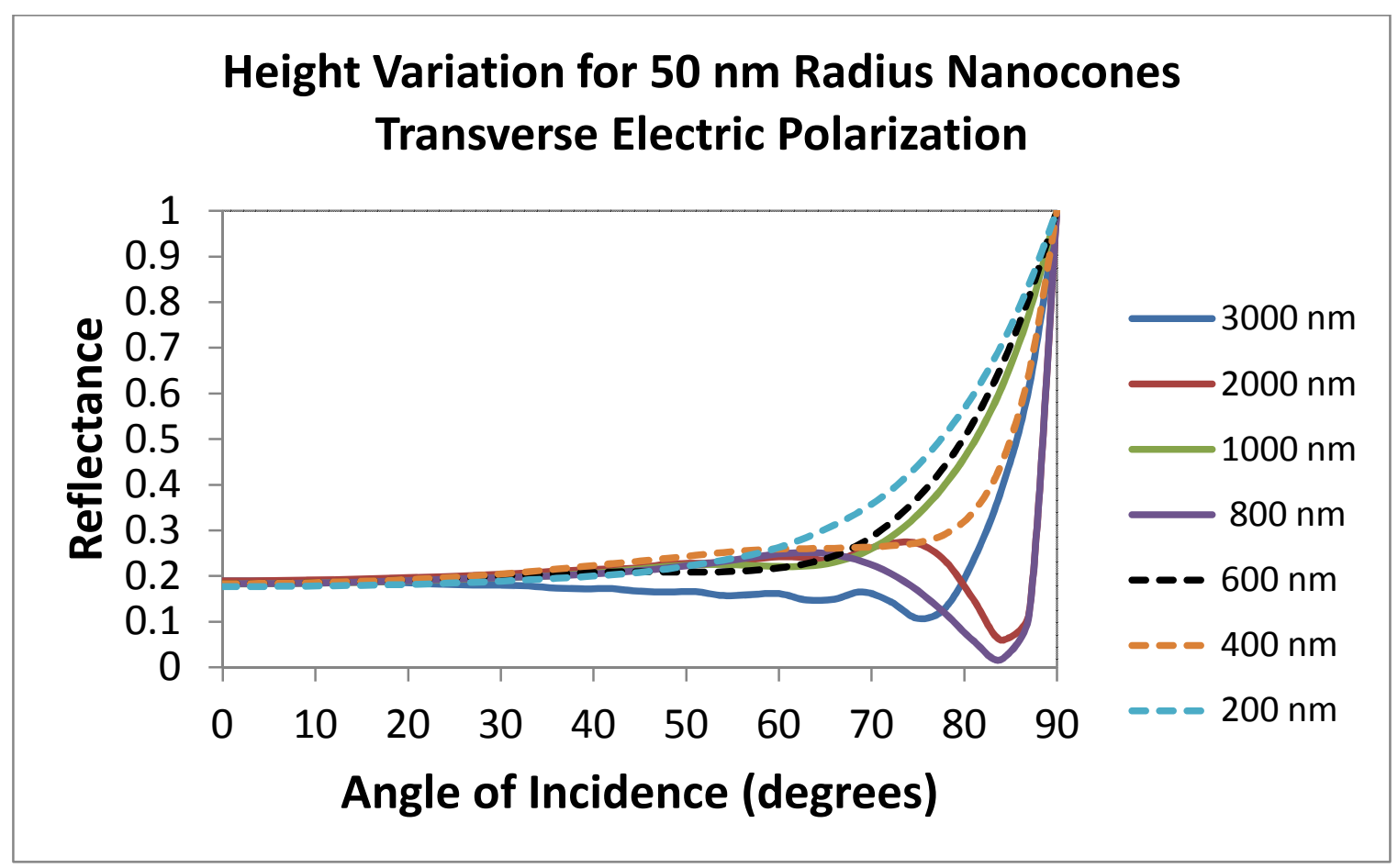

Figure 4.8. Reflectance vs. angle of incidence for varying nanocone heights with fixed radii of $50 \mathrm{~nm}$ for transverse electric fields. Wavelength: $500 \mathrm{~nm}$, Refractive index: Zno 2.0516, Si 4.29749.

Overall, as the height increases, the reflectance decreases in some cases and increases in others. For example, the $400 \mathrm{~nm}$ nanocones for TM polarization show the smallest reflectance, but an average reflectance for the TE case when compared to the others. Figures 4.7 and 4.8 show the height variations for TE mode and TM mode. The shortest structure models showed an increase in reflectivity relative to the $1000 \mathrm{~nm}$ length for TE, with the previous noted exception, while the longer structures generally reduced reflection. Yet, the TM case is quite different with longer structures not showing a reduced reflectance. The $800 \mathrm{~nm}$ heights showed the lowest reflectance when both polarizations were taken into account and both polarizations showed the near-zero dip at high angles. When simulations with wavelengths of $450 \mathrm{~nm}$ and $700 \mathrm{~nm}$ were run, the 
$1000 \mathrm{~nm}$ nanocone model displayed the lowest reflectance. Figure 4.7 shows the height variation reflectance plots for TE mode and TM mode.

\subsubsection{Width Variation}

Widths of the nanocones were varied by increasing the radii from $25 \mathrm{~nm}$ to $100 \mathrm{~nm}$. The limits were set to mimic the laboratory limitations on diameter and to minimize the possibility of computer crashes, due to memory limitations, which increase with enlarging the number of volumetric mesh elements. The heights are fixed at 1 micrometer. The nanocone spacing in the array is not varied, but the distance from the central point of one cone to its neighbor is affected by their radii increasing. A fixed density of a two nanocone radii distance between the neighboring cones centers was used regardless of width. A size comparison of the radii for the nanocone models is shown in figure 4.9.

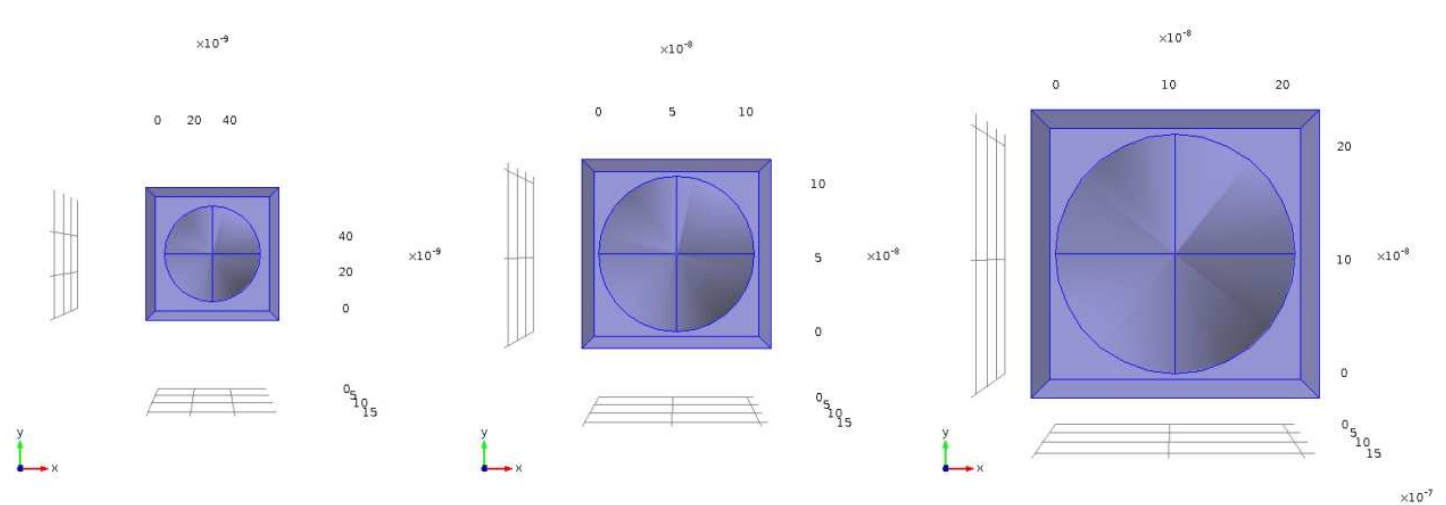

Figure 4.9. Comparison of nanocone radii with left: $25 \mathrm{~nm}$, center: $50 \mathrm{~nm}$, and right: $100 \mathrm{~nm}$. The nanocone models show the top portion of the unit cell including the substrate on which the cones are set and the periodic boundaries. 


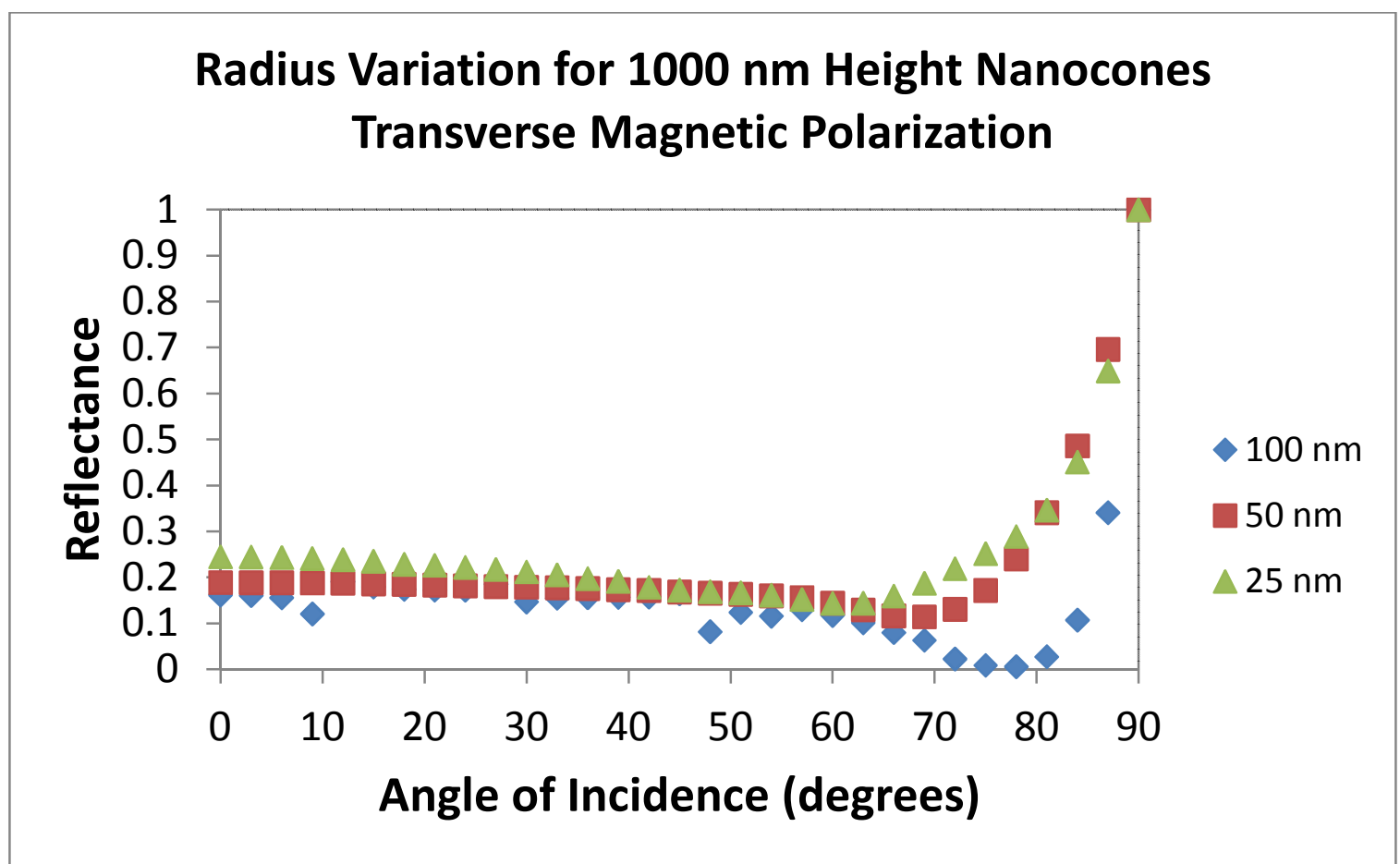

Figure 4.10. Reflectance vs. angle of incidence for nanocone geometries of differing widths and constant $1000 \mathrm{~nm}$ height for transverse magnetic fields. Wavelength: $500 \mathrm{~nm}$, Refractive index: Zno 2.0516, Si 4.29749.

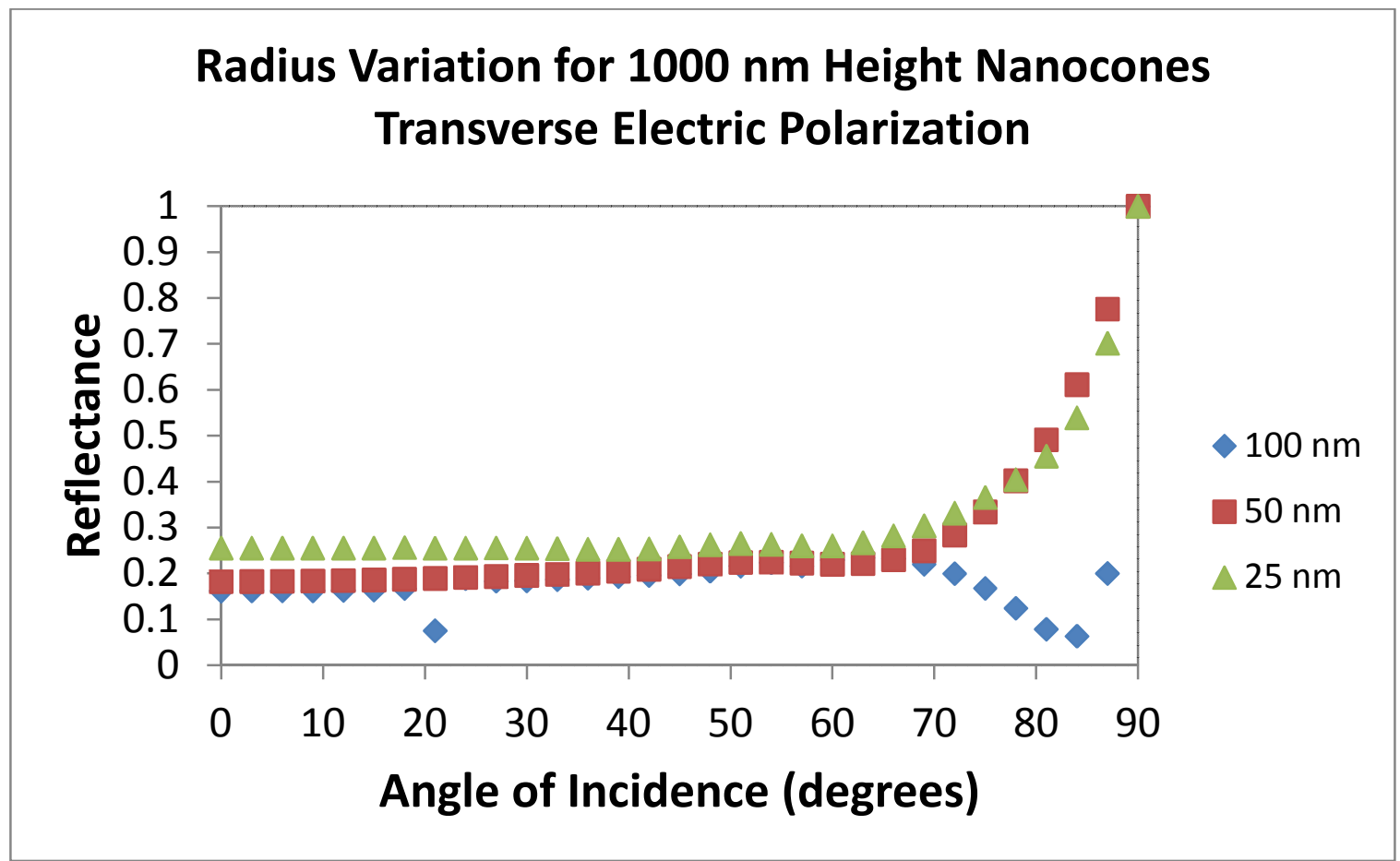

Figure 4.11. Reflectance vs. angle of incidence for nanocone geometries of differing widths and constant $1000 \mathrm{~nm}$ height for transverse electric fields. Wavelength: $500 \mathrm{~nm}$, Refractive index: Zno 2.0516, Si 4.29749. 
The reflectance plots as a function of incident angle in figures 4.10 and 4.11 show the reflectivity decreasing with increasing radius. A larger radius allows the incident light to be transmitted at angles larger than $70^{\circ}$ better than structures with smaller radii. The refracted waves are directed down the larger radii nanocones but get scattered off the tops of the steeper cones. At larger angles, a 10:1 height to radius ratio refracts the light toward a position inside the cone which better guides the light after subsequent refractions than the $20: 1$ or $40: 1$ ratios.

\subsubsection{Density Variation}

The density variation was modeled by adjusting the separation distance between each nanocone center. The nanocone array density affects the transition from air to the substrate as a function of the gradient index profile. Increasing the density theoretically smoothens the transition, provided the cones are spaced enough to allow for conical morphology to be present. The spacing is a function of the unit cell area for fixed cone sizes. As the cell area is increased for a constant radius cone, the density decreases. If the unit cell is square-based, the maximum density is achieved by having the cone's radius equal to half the length of the unit cell's square base. Maximum density is a problem for the simulation since the number of mesh elements increases dramatically with the required decreases in element size needed to fill the small spacing at the wall of the cell where it meets the cone's base. Despite building a more powerful computer to handle such issues, the meeting point of the cone base and the cell wall creates a singular point in the model which cannot be solved for since the solutions cannot be calculated without 
all points remaining inside the geometry. The point can either be removed by a Boolean subtraction or the cone can be shifted into the center of a slightly larger cell area. The latter was chosen with an additional area of $5 \mathrm{~nm}$ added to the unit cell's base, with 2.5 nanometers on each side. This was deemed computationally-sufficient, without compromising the solution, since the amount was two orders of magnitude below the incident wavelength and the nanocone's geometric dimensions. In addition, the nanocone base only fills a circular portion of the cell's square base and the extra spacing is insignificant when compare to the unfilled area.

The density variation (figure 4.12) included a cell structure equal to the width of the cone, with the previous restrictions, a cell length equal to 1.5 times the cone diameter, and twice the cone diameter.

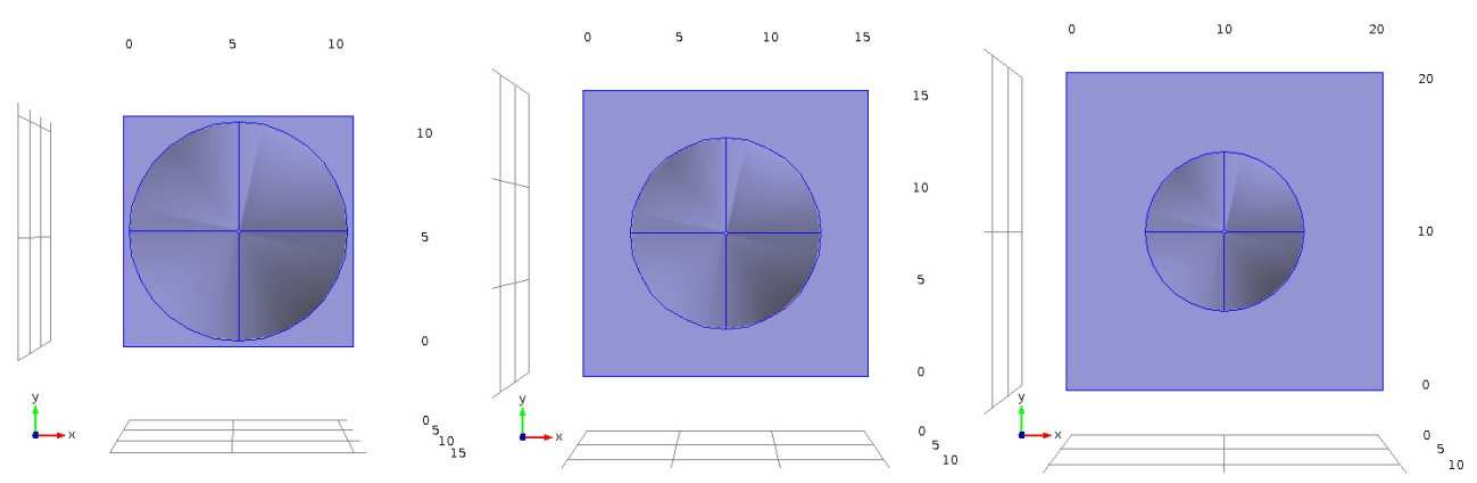

Figure 4.12. Model domains for $\sim 0 \mathrm{~nm}$ spacing, $50 \mathrm{~nm}$ spacing, and $100 \mathrm{~nm}$ spacing between the nanocone bases.

The best performing model is that with no separation between the cones. Only at high angles of incidence, $\sim 70^{\circ}$ and above, does the $50 \mathrm{~nm}$ cone spacing model have a lower reflectivity 


\section{Density Variation \\ TM Polarization}

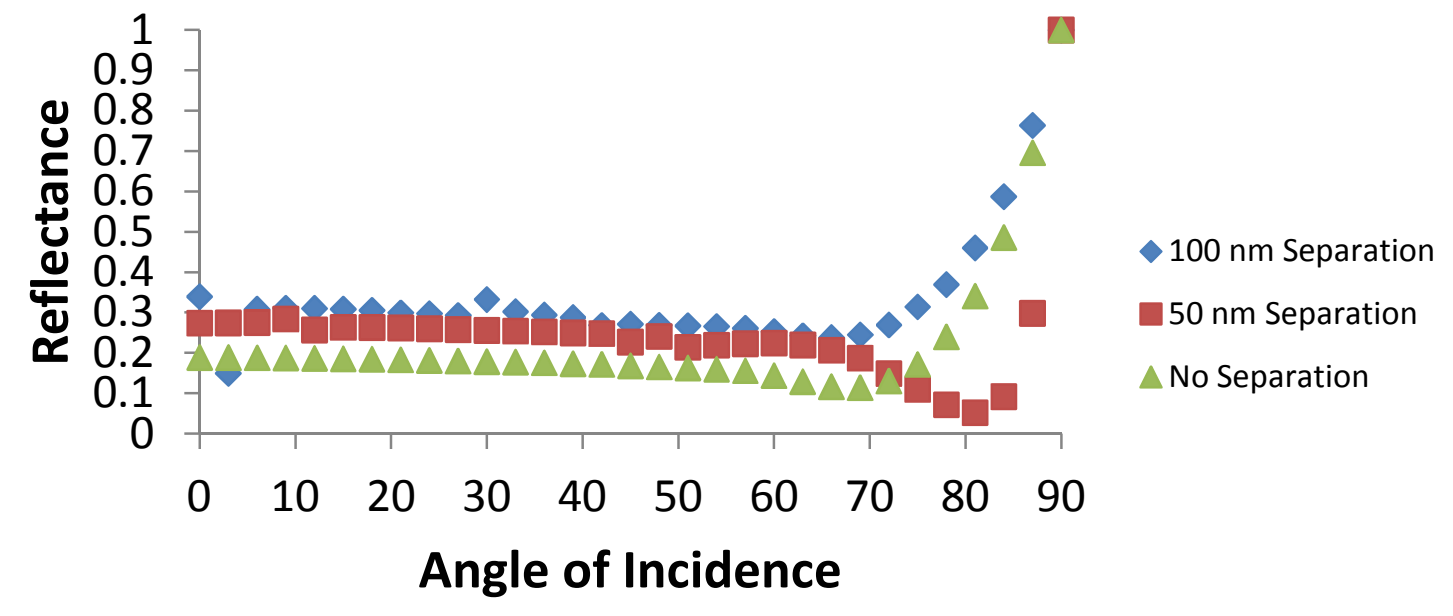

Figure 4.13. Reflectance vs. angle of incidence (TM) plots for nanocone density variations of $100 \mathrm{~nm}$, $50 \mathrm{~nm}$, and $\sim 0 \mathrm{~nm}$ separation distances at the cone bases. Wavelength: $500 \mathrm{~nm}$, Refractive index: Zno 2.0516, Si 4.29749.

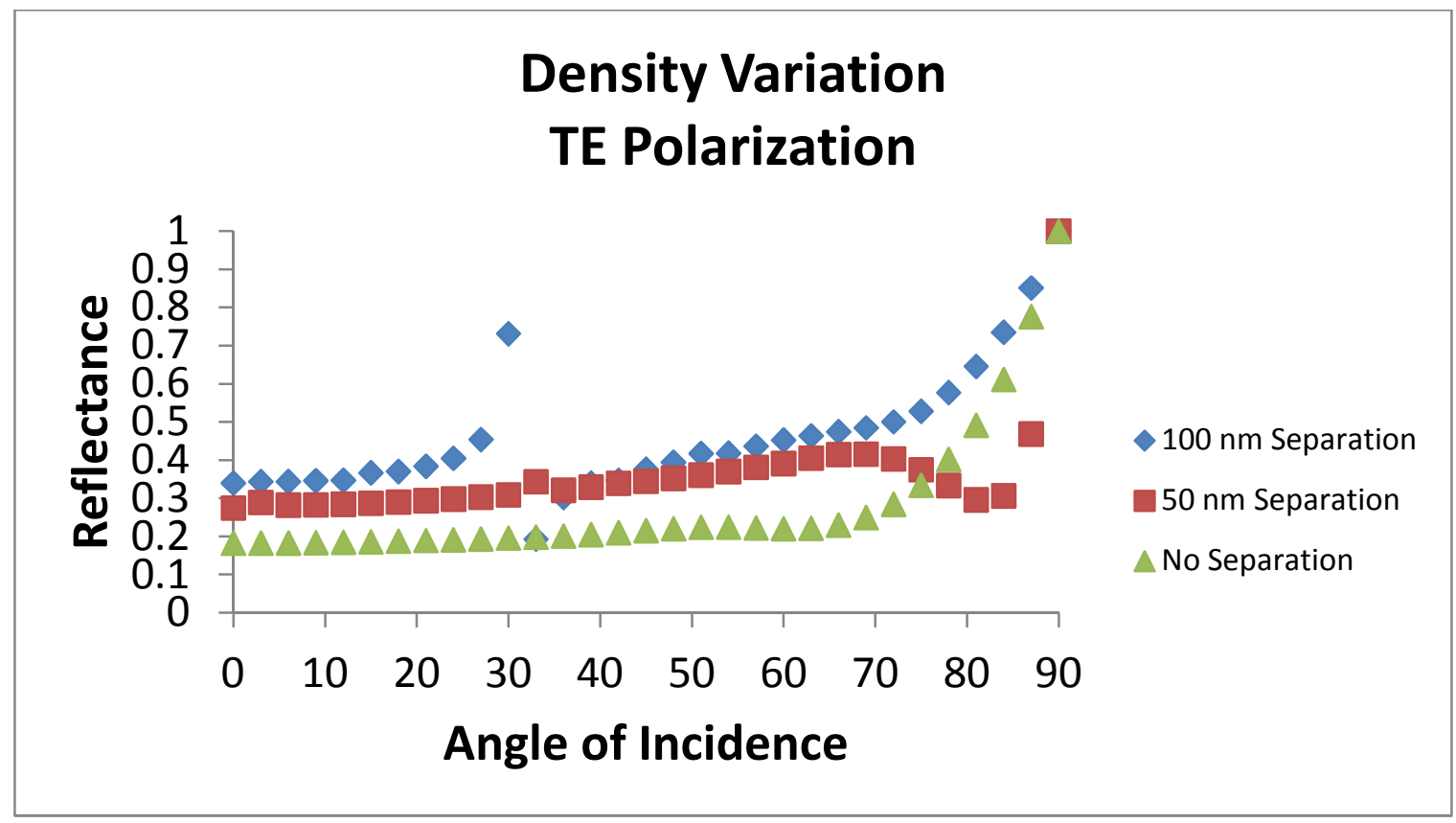

Figure 4.14. Reflectance vs. angle of incidence (TE) plots for nanocone density variations of $100 \mathbf{n m}$, $50 \mathrm{~nm}$, and $\sim 0 \mathrm{~nm}$ separation distances at the cone bases. Wavelength: $500 \mathrm{~nm}$, Refractive index:

Zno 2.0516, Si 4.29749. 


\subsection{Nanocone Comparison with Quintic Profile Gradient Index Films}

The quintic profile provides the benefit of very slow transitions of the refractive index near the interfaces. Since the quintic profile remains the highest standard for gradient index anti-reflective material, comparisons with the profile are made with varying incident angle and wavelength [41]. The profile is compared with a $1000 \mathrm{~nm}$ height $\mathrm{x} 50$ $\mathrm{nm}$ radius nanocone model, as was shown in figure 2.5 and is repeated here in figure

\subsection{5.}

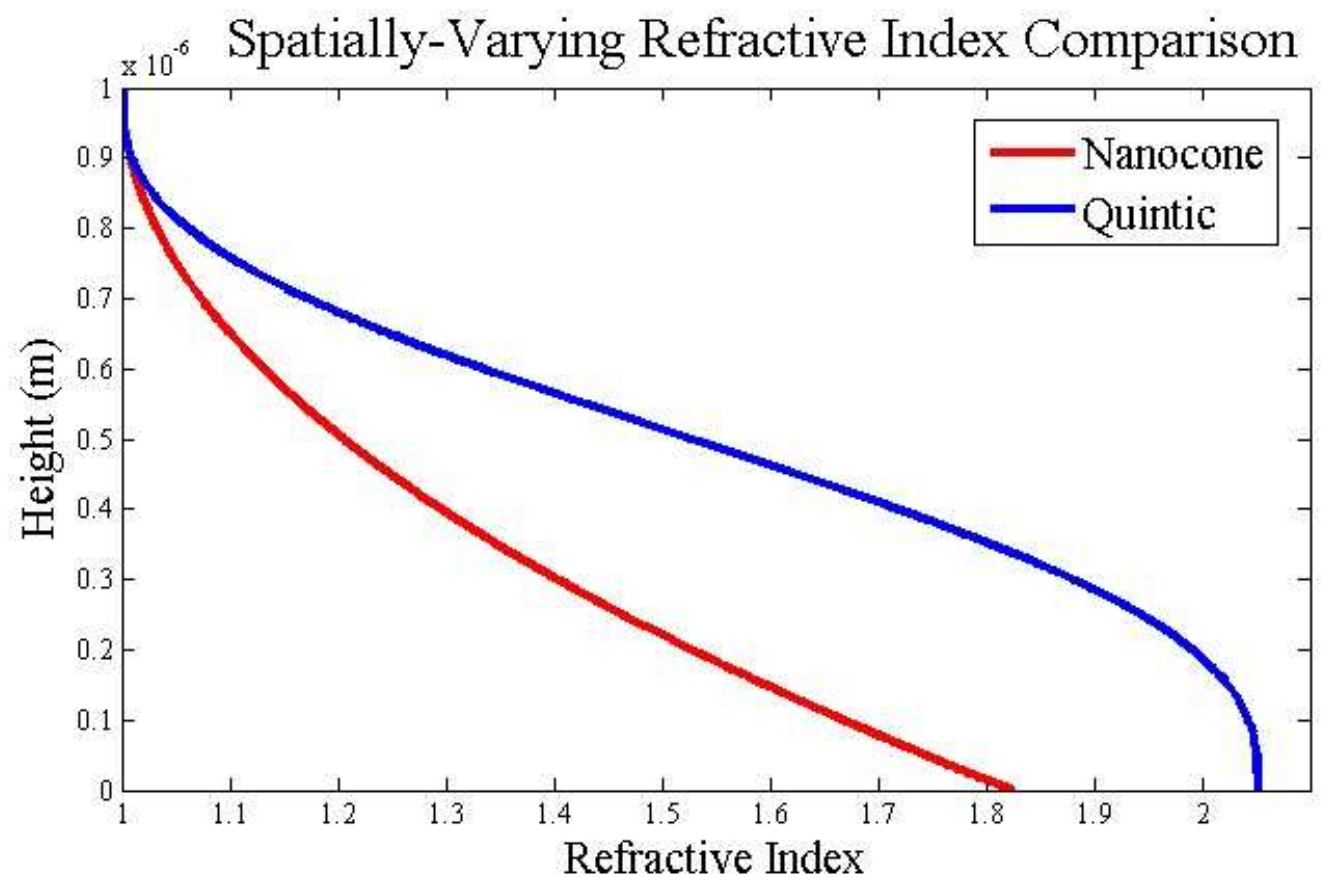

Figure 4.15. Spatially-varying refractive index comparison for nanocones and the quintic profile.

\subsubsection{Angular Dependence}

The incident angle was swept from perpendicular to $90^{\circ}$ in increments of $3^{\circ}$. The wavelength is fixed at $63 \mathrm{~nm}$ for the incident light. Both the nanocone and quintic models are high-performing for a wide variety of incident angles. 

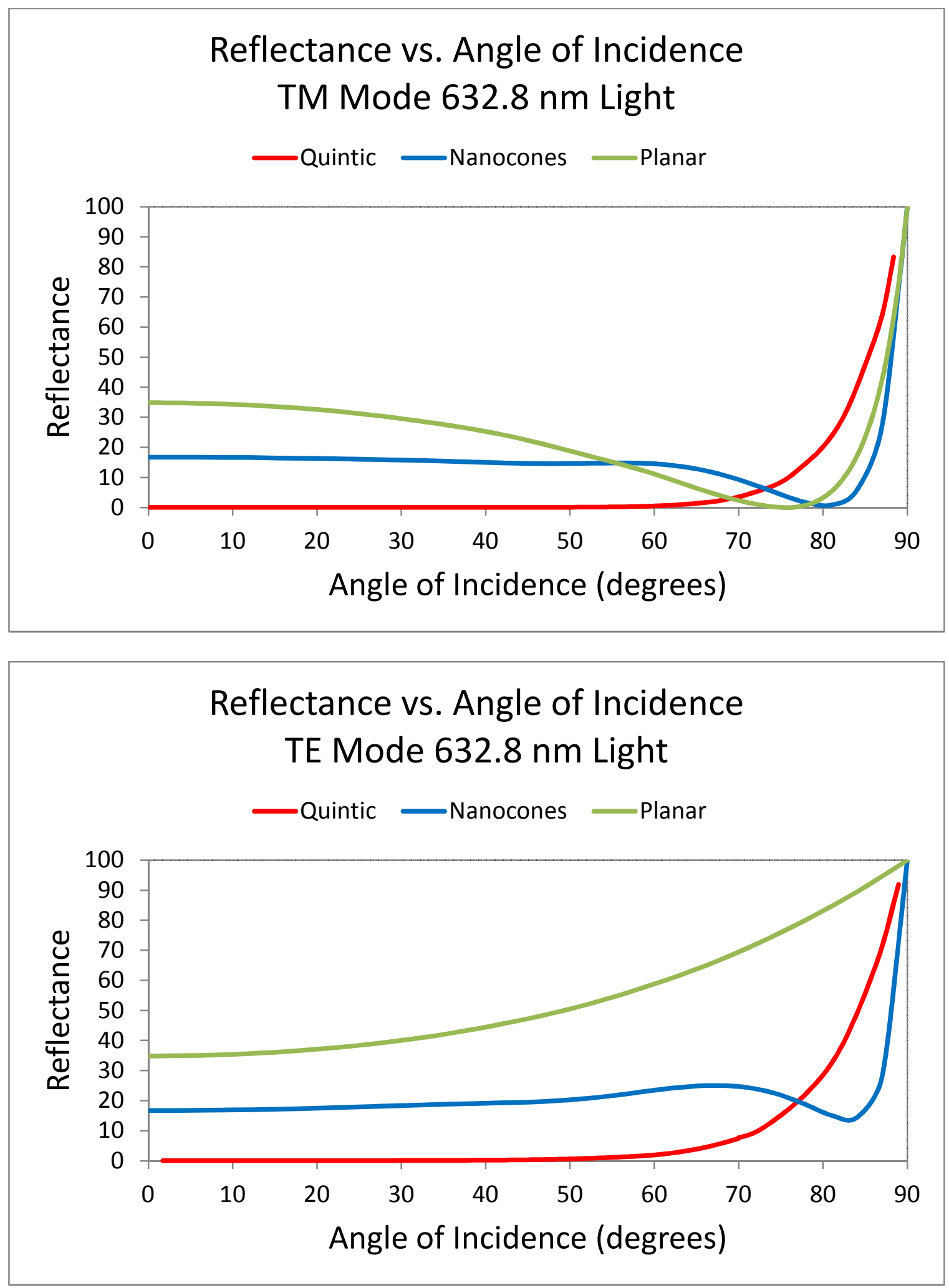

Figure 4.16. Reflectance comparison for quintic profile, planar, and $1000 \mathrm{~nm}$ height $x 50 \mathrm{~nm}$ radius nanocones for TM (top) and TE (bottom) at $632.8 \mathrm{~nm}$. Si index is 3.88163 and $\mathrm{ZnO}$ index is 1.98882 . 
The quintic profile exhibits near zero reflectance for many incident angles. The nanocone model is about half way between this reflectance and a planar interface model. For angles greater than $75^{\circ}$, with TM or TE polarization, the nanocone model outperforms the quintic profile gradient media model.

\subsubsection{Wavelength Dependence}

Although some nanocone models performed better than others for varying incidence, with a fixed wavelength of $500 \mathrm{~nm}$, this limits the solar spectrum to its maximum only, but discounts the remaining wavelengths. The electromagnetic radiation modeled ranges from $450 \mathrm{~nm}$ to $700 \mathrm{~nm}$ which mimics the visible portion of the spectrum.

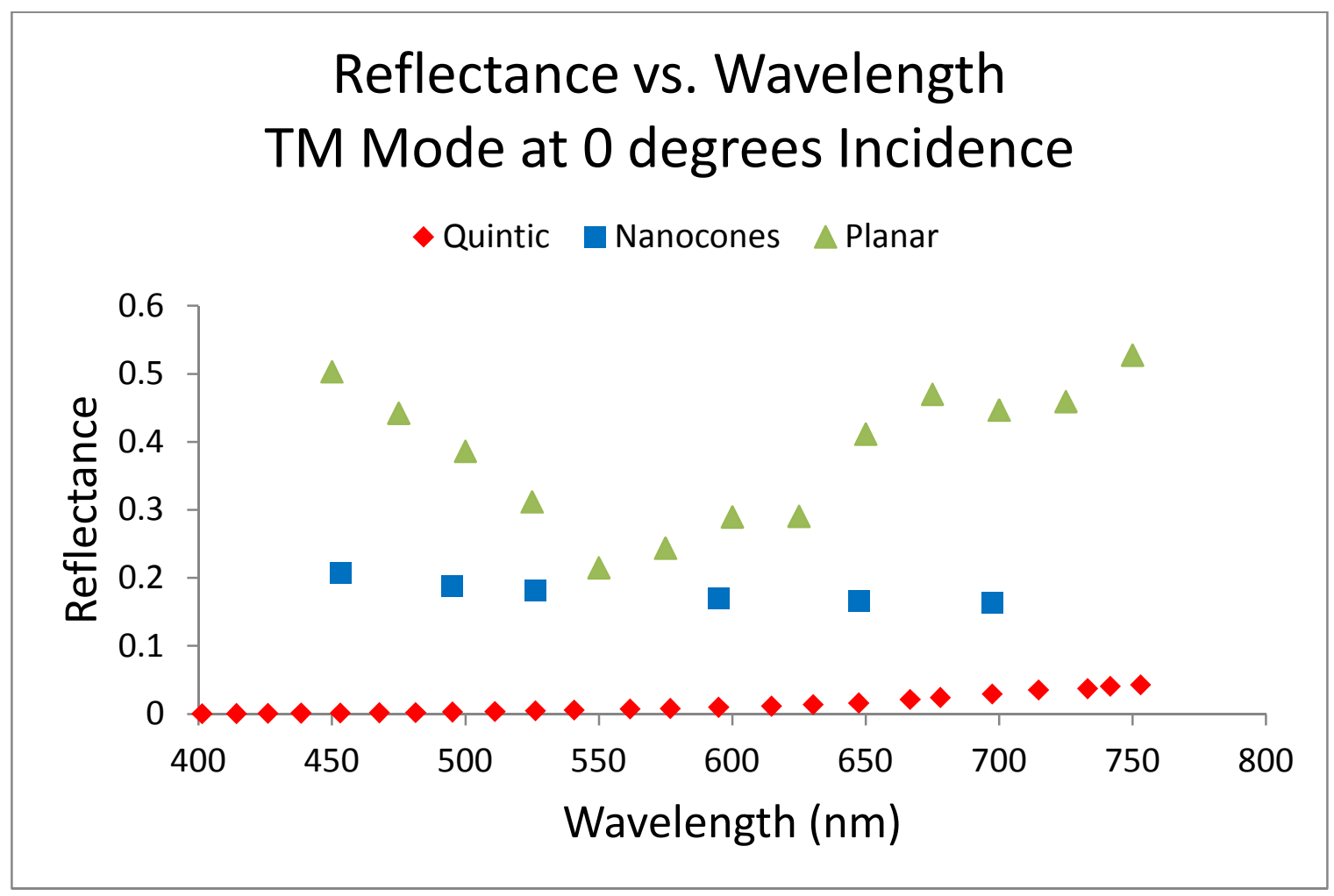

Figure 4.17. Reflectance comparison for quintic profile media and $1000 \mathrm{~nm}$ height $x 50 \mathrm{~nm}$ radius as a function of wavelength with perpendicular incidence TM light. 


\section{Reflectance vs. Wavelength TE Mode at 0 degrees Incidence}

$\diamond$ Quintic Nanocones $\triangle$ Planar

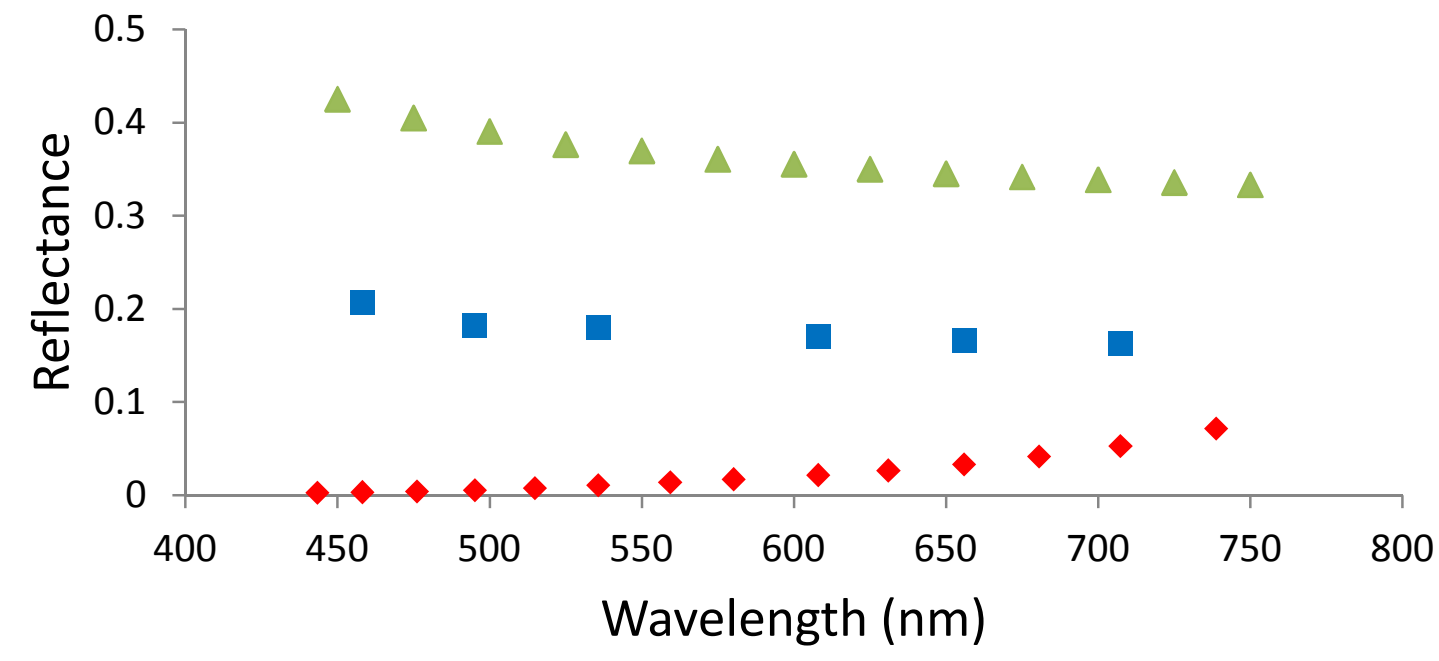

Figure 4.18. Reflectance comparison for quintic profile media and $1000 \mathrm{~nm}$ height $\times \mathbf{5 0} \mathrm{nm}$ radius as a function of wavelength with perpendicular incidence TE light.

\subsection{Quasi-Randomized Nanocone with Periodic Unit Cells}

To more accurately model the structures created in a laboratory setting, a quasi-random unit cell was created containing 10 nanocones. The heights, radii, tilt, surface position, and apex truncation were all varied to maximize the randomness. Still, since periodicity was used to model a much larger area than the initial unit cell, the geometry is only quasirandom due to the periodicity of the cell in the xy-plane. The incident angle is varied and the wavelength of light is $500 \mathrm{~nm}$. 
The model is constructed from a unit cell with $375 \mathrm{~nm}$ depth and width for the silicon substrate dimensions. The depth is $500 \mathrm{~nm}$ but has a back surface with absorbing boundary conditions. This makes the silicon substrate effectively infinitely thick to minimize internal reflections due to the simulation parameters and not the physical model. Within the unit cell the randomization of the zinc oxide conical structures included variations in vertical direction, diameter, and placement. The corresponding parameters are summarized in table 4.1 and the geometry is displayed in figure 4.19. This 10 cone orientation presents a computationally-demanding model due to the extreme variance in the structures and the high number of degrees of freedom and mesh elements needed.

Table 4.1. Randomization parameters for 3D quasi-random nanocone model.

\begin{tabular}{|l|l|l|l|l|l|}
\hline $\begin{array}{l}\text { Cone } \\
\text { Number }\end{array}$ & $\begin{array}{l}\text { Position } \\
(\mathrm{x}, \mathrm{y})(\mathrm{nm})\end{array}$ & Height $(\mathrm{nm})$ & Radius $(\mathrm{nm})$ & $\begin{array}{l}\text { Tilt }(\mathrm{nm}) \\
(\mathrm{x}, \mathrm{y})\end{array}$ & $\begin{array}{l}\text { Top/Bottom } \\
\text { Ratio }\end{array}$ \\
\hline 1 & $(57.5,57.5)$ & 800 & 50 & $(0,-25)$ & 0.1 \\
\hline 2 & $(167.5,82.5)$ & 1100 & 60 & $(+25,12.5)$ & 0.05 \\
\hline 3 & $(287.5,57.5)$ & 1000 & 50 & $(0,+50)$ & 0.1 \\
\hline 4 & $(145,205)$ & 850 & 60 & $(-25,0)$ & 0.05 \\
\hline 5 & $(57.5,150.5)$ & 900 & 40 & $(+25,0)$ & 0.1 \\
\hline 6 & $(287,190)$ & 1150 & 70 & $(-25,+25)$ & 0.1 \\
\hline 7 & $(60,315)$ & 1000 & 50 & $(-12.5,12.5)$ & 0.05 \\
\hline 8 & $(190,315)$ & 1000 & 55 & $(12.5,-12.5)$ & 0.075 \\
\hline 9 & $(300,315)$ & 850 & 45 & $(-12.5,-12.5)$ & 0.05 \\
\hline 10 & $(50,225)$ & 750 & 30 & $(0,0)$ & 0.025 \\
\hline
\end{tabular}

The mesh elements needed to be sufficiently small, ideally one-tenth the incident wavelength, to allow for accurate calculations and to properly discretize the sharp corners of the cones which produce small areas needing a high density mesh. The desired mesh needed to be adjusted to allow the calculations to be performed in a reasonable amount of 
time. Sectional meshing, which decreased the total number of elements by user-defined face and boundary elements, enabled an appropriate sizing while ensuring computability. The type and quantity of the elements are listed in table 4.2.

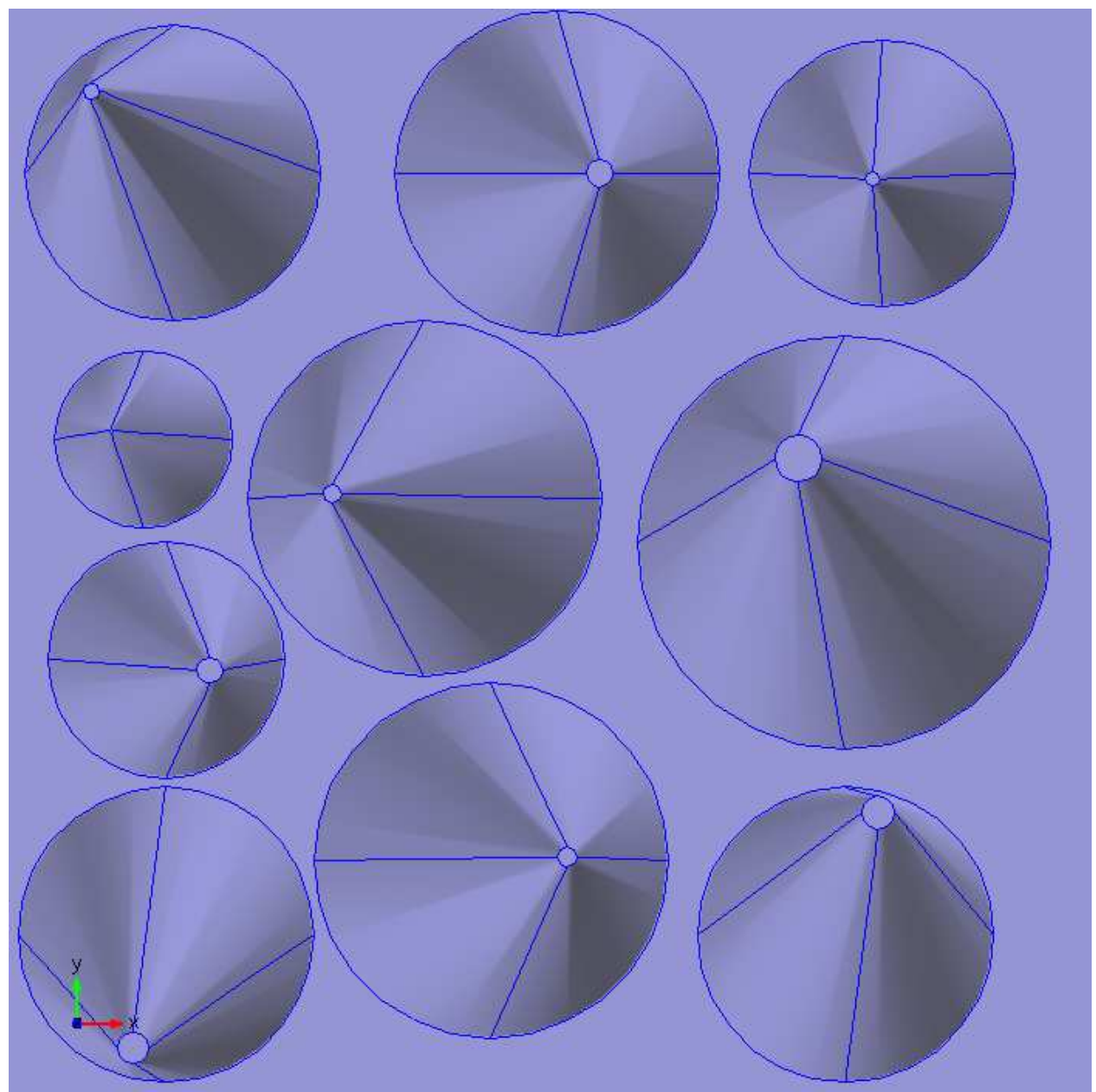

Figure 4.19. Top view of 3D quasi-randomized nanocone model. Coloring for contrast.

Table 4.2. Type and quantity of 3D quasi-random nanocone model mesh elements.

\begin{tabular}{|l|c|c|c|c|}
\hline & Point & Edge & Boundary & Volume \\
\hline $\begin{array}{l}\text { Number of } \\
\text { Mesh Elements }\end{array}$ & 92 & 5594 & 50,492 & 692,006 \\
\hline
\end{tabular}


These mesh element numbers are significantly smaller than the originally attempted refinement. With the desired scaling of the mesh size with geometry, wavelength, and dielectric properties, the mesh elements were an order of magnitude higher and made computation impossible due to the computer server's memory limitations. Even with much fewer mesh elements, the models have minimal artifacts in the solutions. The mesh for the top view of the geometry from figure 4.19 is shown in figure 4.20.

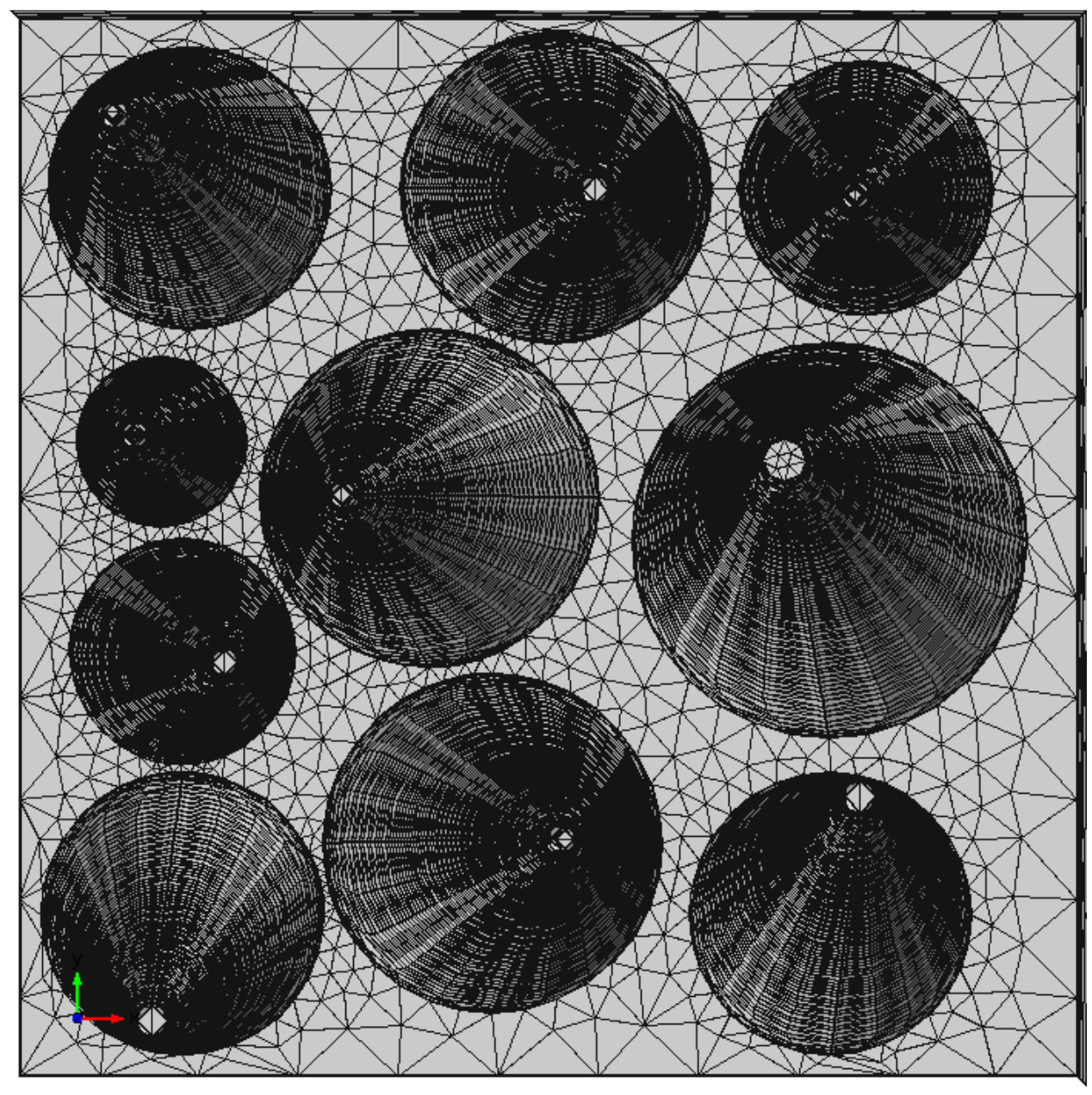

Figure 4.20. Top view of 3D quasi-randomized nanocone model geometry and mesh. 
A $45^{\circ}$ view, with-respect-to the xy-plane, is displayed in figure 4.21 . The increased number of mesh elements can be seen at the cone bases and peaks. The triangular shapes of the mesh elements on the exterior surfaces are created separately from the interior tetragonal structures. The surface meshing allows for proper computation of the fields at the boundaries in a model with larger interior tetragonal elements.

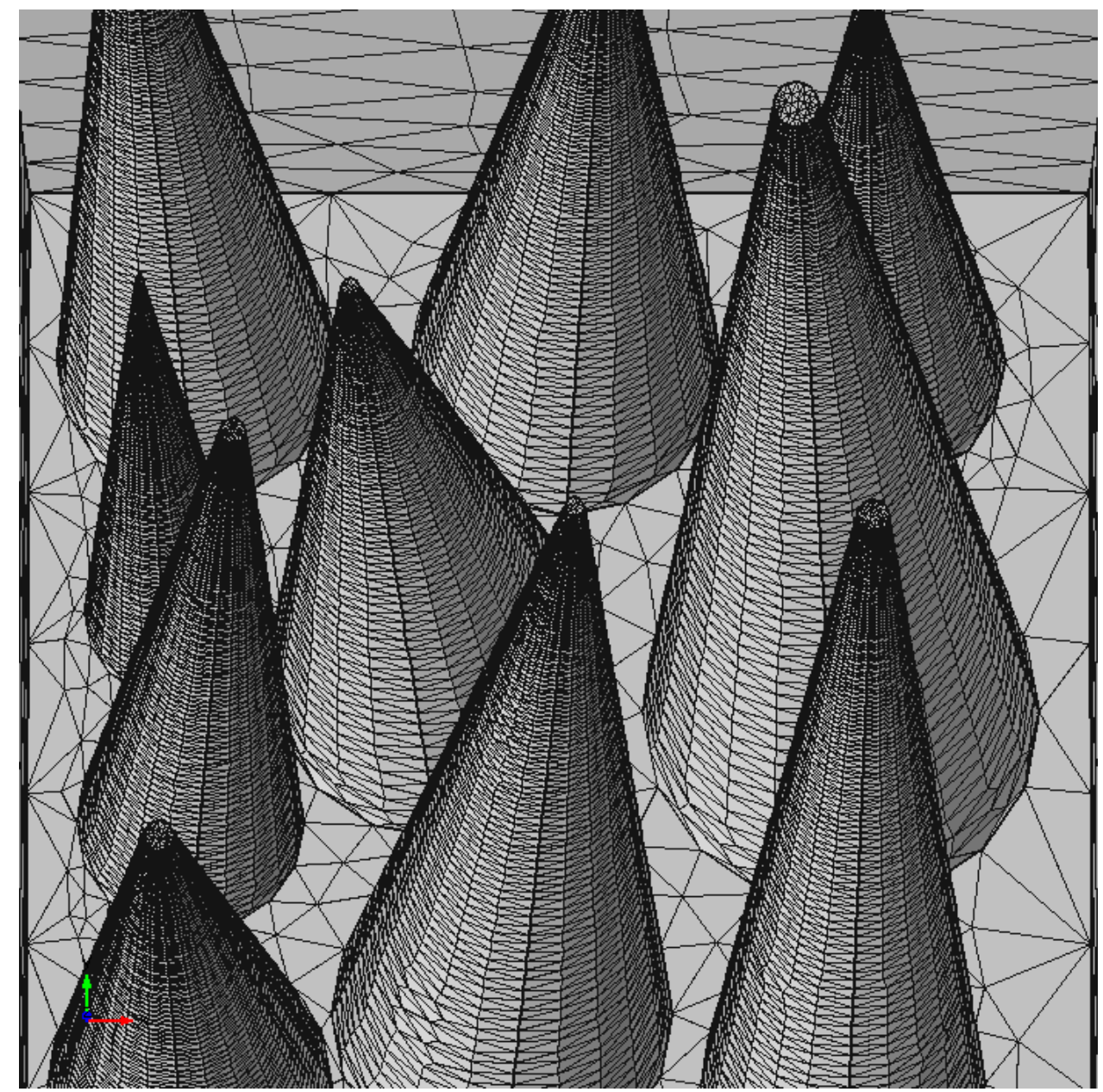

Figure 4.21. $45^{\circ}$ from $x y$-plane view of $3 \mathrm{D}$ quasi-randomized nanocone model geometry and mesh.

Figure 4.22 displays the TM (left) and TE (right) polarization models with the field solutions for the magnetic and electric fields at a $45^{\circ}$ incident angle. The randomized 
nature of the cones increase the light propagation directional dependence over verticallyaligned cones. Vertical conical shapes are azimuthally-symmetric in the nanocone array and this guides the wave into the substrate. The light path randomization in the quasirandom model is enhanced upon each successive scattering event. The reflectance plot shows a decrease over planar interfaces for both transverse magnetic and transverse electric propagation by a factor of two or more, but the reflection is higher than with the vertical alignment.
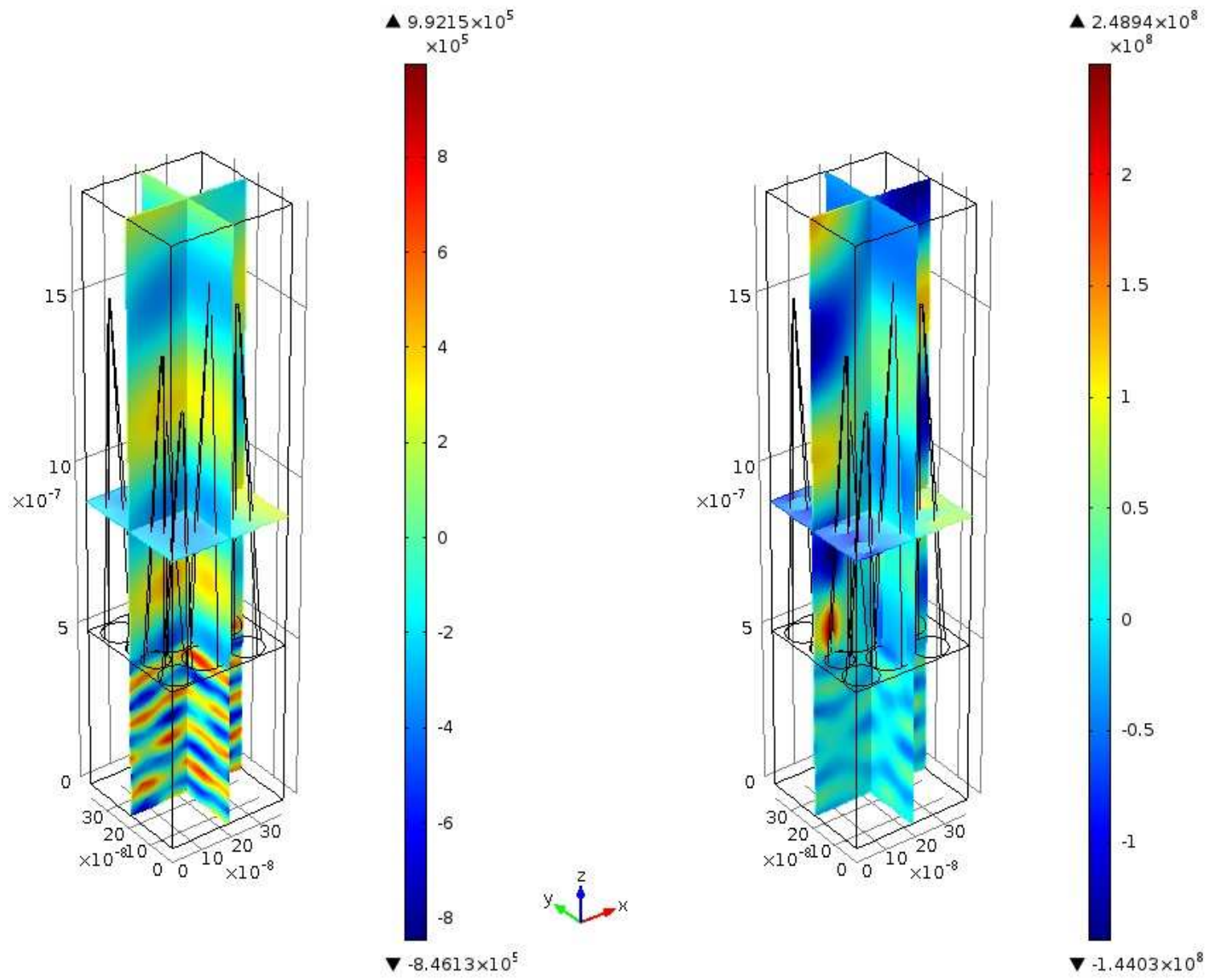

Figure 4.22. The field solutions at a $45^{\circ}$ angle for the magnetic field (left) in transverse magnetic polarization and electric field (right) in transverse electric polarization. 
The curves follow a similar pattern to the one followed by the single nanocone array, with a slight shift toward higher values. The reflectivity has been decreased from the planar interface values by $\sim 40 \%$ except for high angles of incidence in the TM planar case. The overall improvement was not as significant as in the purely vertical nanocone model. The outlying points for the nanocone model are limited in number and require discussion of their validity. The vertically-aligned, purely periodic nanocone reflectance is shown for comparison.



Figure 4.23. Reflectance vs. angle of incidence plot for 3D quasi-random nanocone model. Both TM and TE propagations for the air/ZnO nanocone/Si substrate model are shown in comparison with a planar interface. Wavelength: $500 \mathrm{~nm}$, Refractive index: Zno 2.0516, Si 4.29749. 


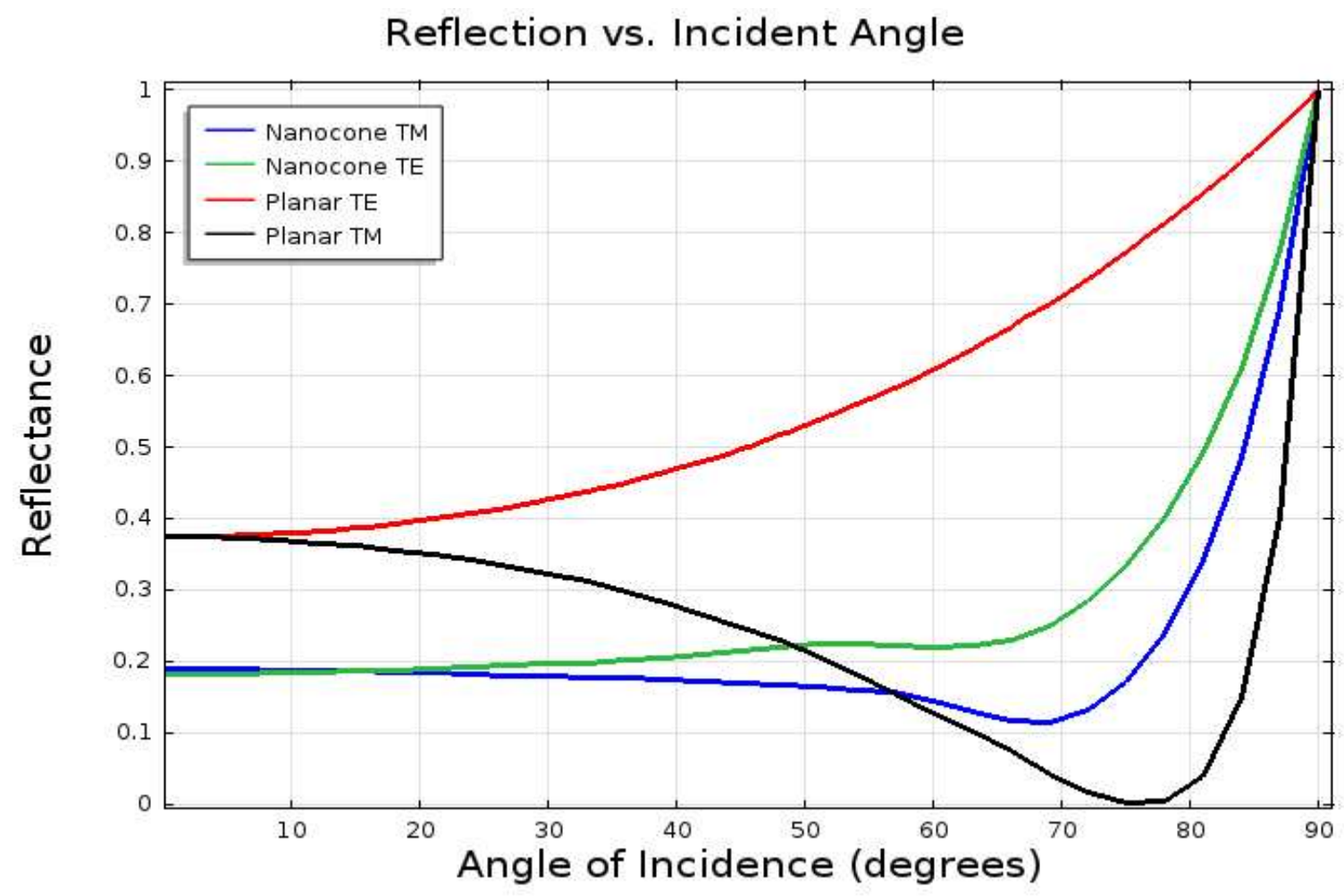

Figure 4.24. Reflectance vs. angle of incidence plot for 3D vertical and periodic nanocone model. Both TM and TE propagations for the air/ZnO nanocone/Si substrate model are shown in comparison with a planar interface. Wavelength: $500 \mathrm{~nm}$, Refractive index: Zno 2.0516, Si 4.29749.

The plots for the reflectance of the quasi-random nanocone models (figure 4.23) contain a few outlying points and some scattering of data. This was caused by the need for further refinement of the mesh. Mesh elements could not be made smaller without causing a crash of the server due to memory limitations. Several attempts to find the optimal mesh size were conducted. The elements needed to be small enough to yield a reasonable solution, yet large enough to allow computation. The elements listed in table 4.2 show the quantities needed to create the mesh. With only a few outliers which are well off the modeled curves, the plot in figure 4.23 displays a satisfactory solution provided the outliers can be proven extraneous. 
The plot in figure 4.23 is a model with mesh element refinement. Previous models had meshes which were not optimized and were still too coarse to give good results. A previous version is shown in figure 4.25 and exhibits the more sporadic data points associated with too coarse of a mesh.

The data points for the TM and TE polarizations are more scattered which represents the difficulty in finding a satisfactory solution with too large of mesh elements. A comparison of the plots in figures 4.23 and 4.25 shows most of the higher valued data points dropping toward the rest of the data points upon refinement. However, the outlier at $39^{\circ}$ for the TE plot remains at a value considered too high to be a reasonable solution. To check the validity of the point, the model was run again $1.5^{\circ}$ above and below the incidence angle with step sizes bisected for each run. This is shown in figure 4.26. The appearance of a sharp peak spiking at $39^{\circ}$ and then rapidly dropping off to the left and right is indicative of an artifact. 


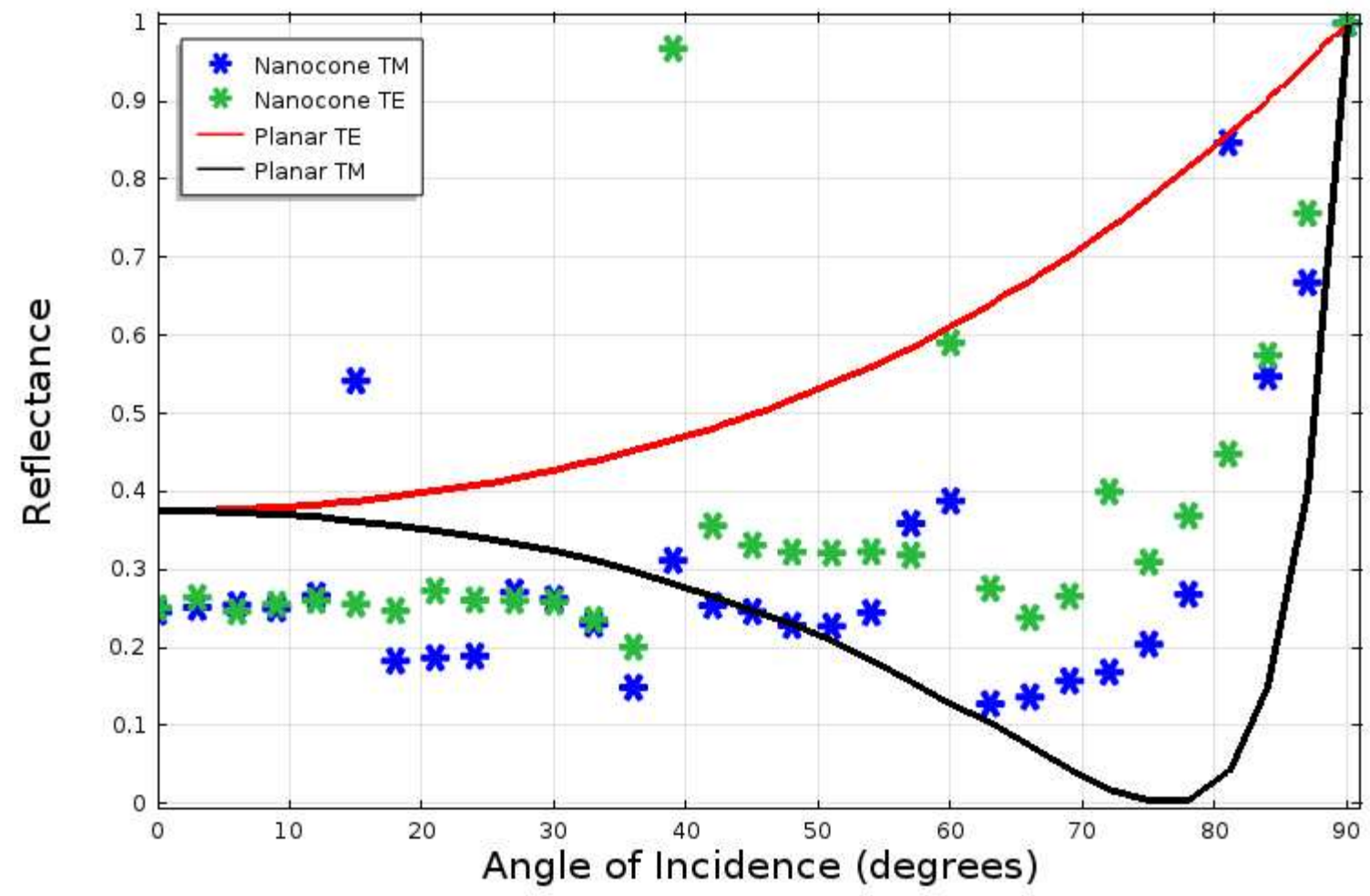

Figure 4.25. Quasi-random nanocone reflectance plot with a coarse mesh and more outliers.

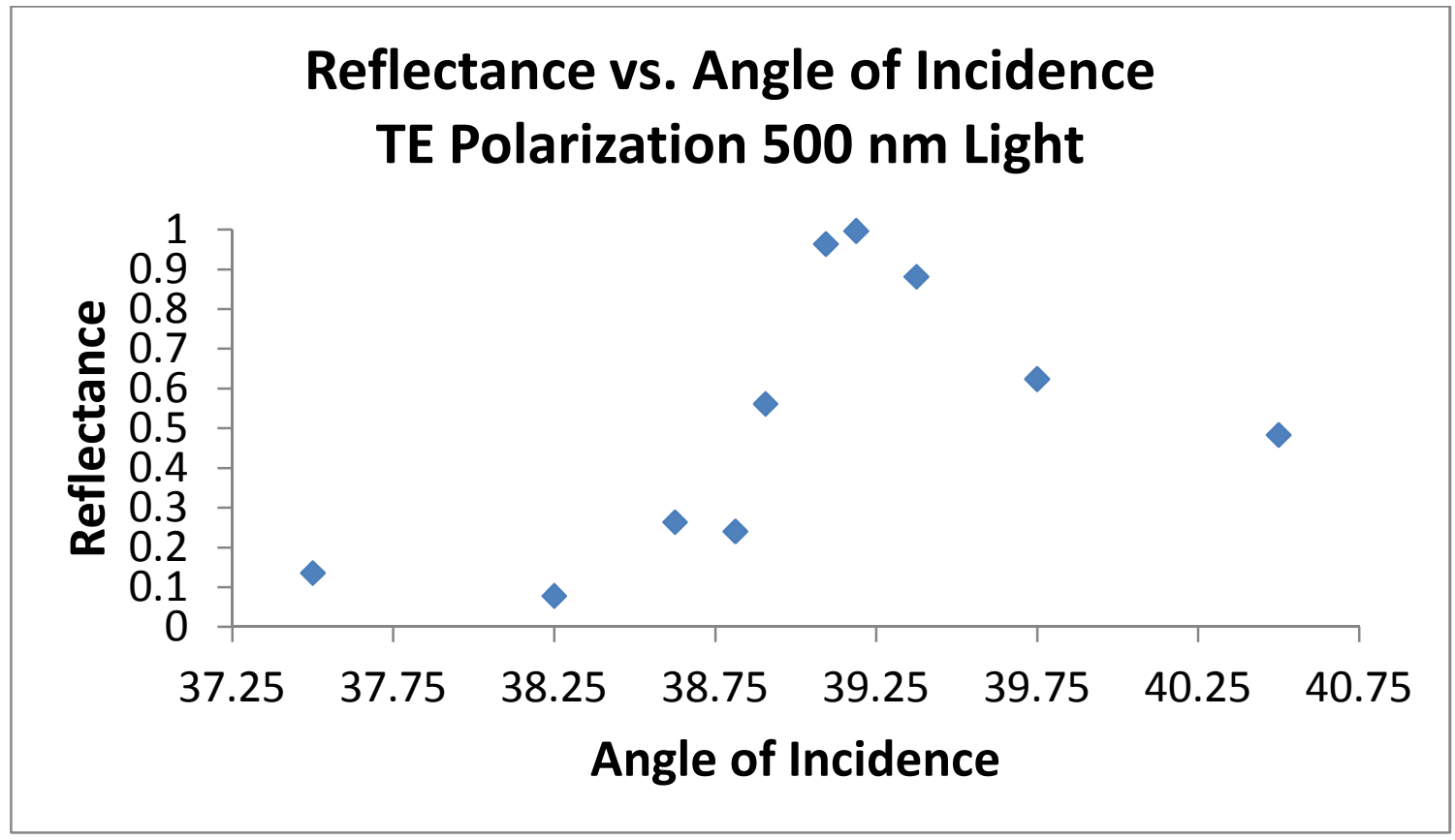

Figure 4.26. Reflectance plot for quasi-random nanocone model with incident angle varying from 37.5 to 40.5 degrees. 
While the outlying point at $39^{\circ}$ appears to be an artifact, a check as to whether or not this was a resonance phenomenon was completed. This seems highly unlikely, since the random nature of the geometry would not easily create such a response. The integrated intensity from the reflected light would have to be very close to $100 \%$ at the top boundary. The geometry of the nanocones has a rounded surface which redirects scattered light from its surface toward varying directions. With cones positioned in a nonuniform manner on the substrate and having differing orientations and sizes, the near total reflectance at a certain angle causing a resonance peak with an amplitude of $100 \%$ is highly unlikely.

\subsection{Seed Layer}

A seed layer can be applied to substrates to enhance growth of nanostructures. Part of the motivation for this thesis were growths of nanowires in the laboratory setting using a seed layer. Spray pyrolysis was used to grow the wires from a zinc oxide seed layer. The average layer thickness was $50 \mathrm{~nm}$ and this size is modeled here. A hexagonal wurtzite crystalline structure formed under these conditions and differs from the conical shape here.

The seed layer effectively smoothens the transition in the refractive index from the $\mathrm{ZnO}$ nanocone/air geometric mixture to the substrate by adding an additional medium of $\mathrm{ZnO}$. The jump in effective refractive index between the nanocones and the substrate is decreased and the index profile better mimics the quintic (ideal) profile. The quintic and 
seed layer nanocone refractive index profiles are plotted in figure 4.27. The quintic profile has an inflection point in the middle of the plot which causes it to turn down at the end point. A seed layer for the nanocone mimics this inflection causing the smoother transition. The constant index of the seed layer better matches the Si substrate index.

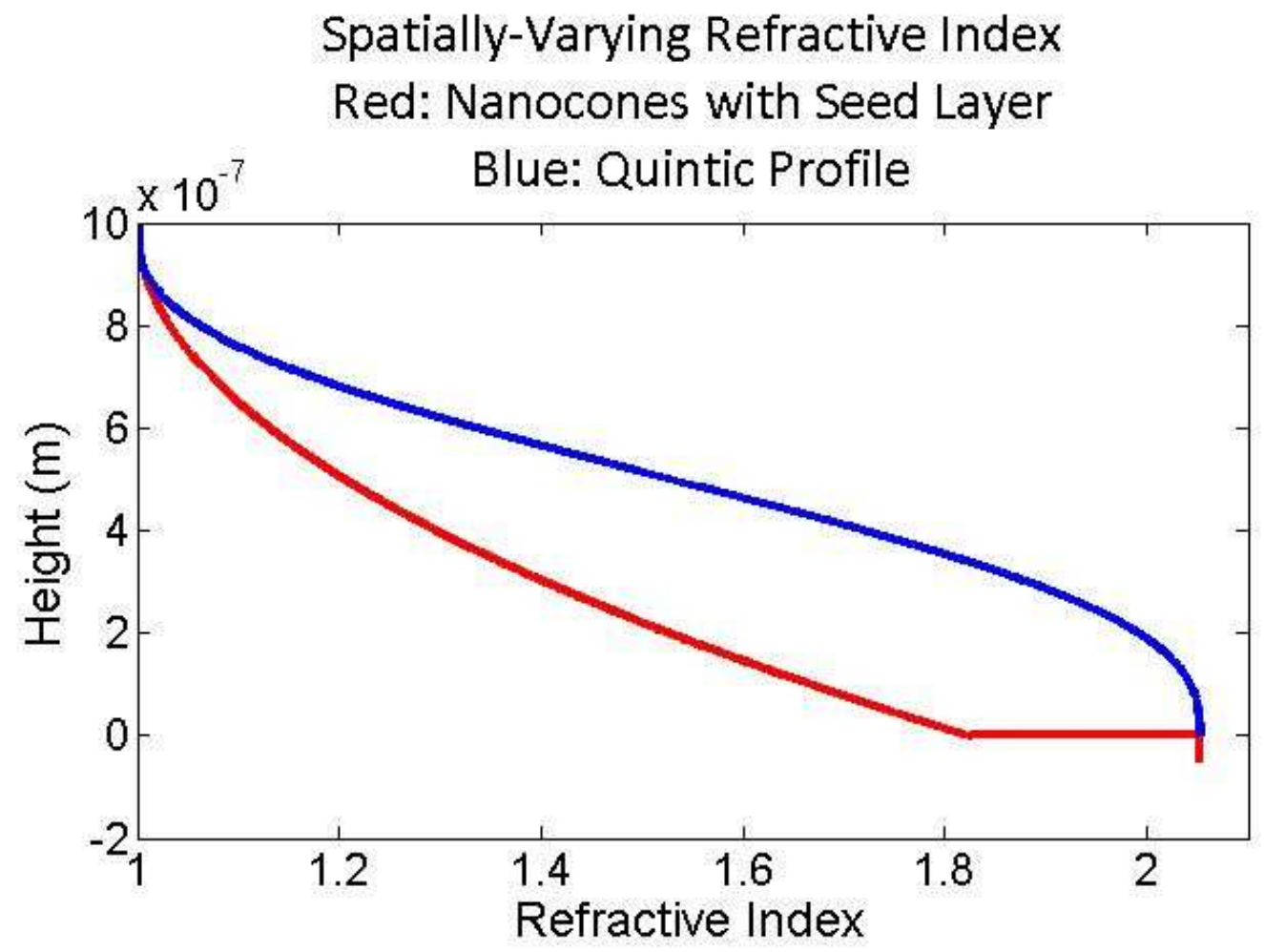

Figure 4.27. Spatially-varying refractive index profiles for nanocones with a $50 \mathrm{~nm}$ seed layer (red, bottom) and the quintic profile (top, blue). The seed layer height is added to the $1000 \mathrm{~nm}$ height of the nanocones. This is shown here as $\mathbf{- 5 0} \mathbf{~ n m}$ height since it is a part of the substrate and does not affect the cones.

The magnetic and electric fields are compared for models with and without a seed layer in figure 4.28 for $1000 \mathrm{~nm}$ tall cones with $50 \mathrm{~nm}$ radii. The overall reflectance decreases significantly by $\sim 50 \%$ (figure 4.29 ). When plotted in comparison with the planar model, a dip in reflectance for both the polarizations mimics the transverse magnetic dip in reflectance near the Brewster angle. This was not seen in most TE cases previously 
discussed. In the model without the seed layer, the TE and TM reflectance curves begin at just under $20 \%$ and are approximately equal until $\sim 18^{\circ}$, at which point, the TM curve starts to dip lower and the TE deviates upward. In the previous model, the TE and TM divert from one another but here they stay relatively close and low in value. The reflectance plots do not start to increase appreciably until a much higher angle of incidence is reached. The plots for with (top) and without (bottom) seed layers are compared in figure 4.29. A comparison with a planar interface is also made in each plot for TM and TE polarizations.
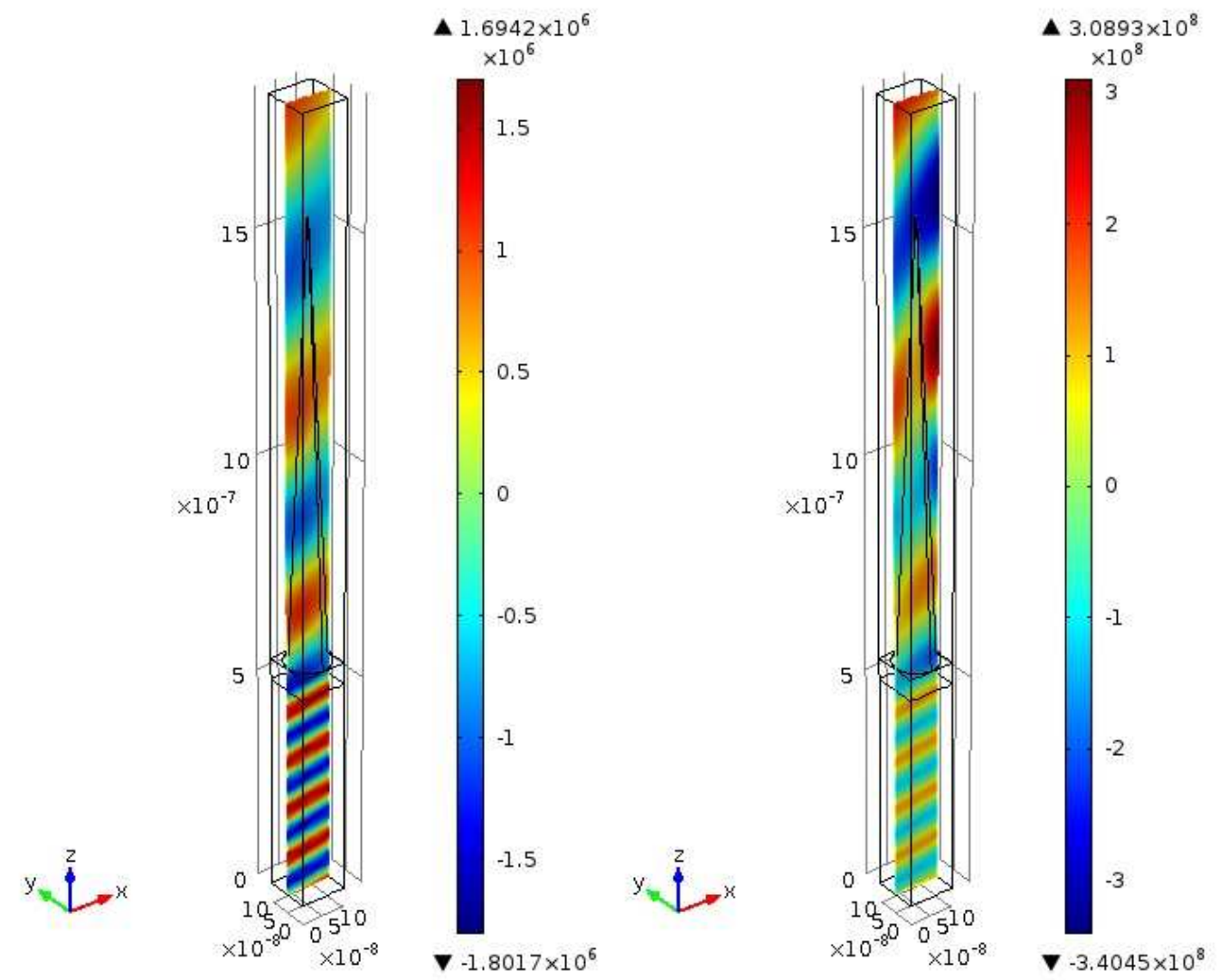

Figure 4.28. $1000 \mathrm{~nm}$ height $\times 50 \mathrm{~nm}$ radius nanocone field plots for the $y$-component with TM (top left) and TE (top right) polarizations. Light is incident at $45^{\circ}$. 

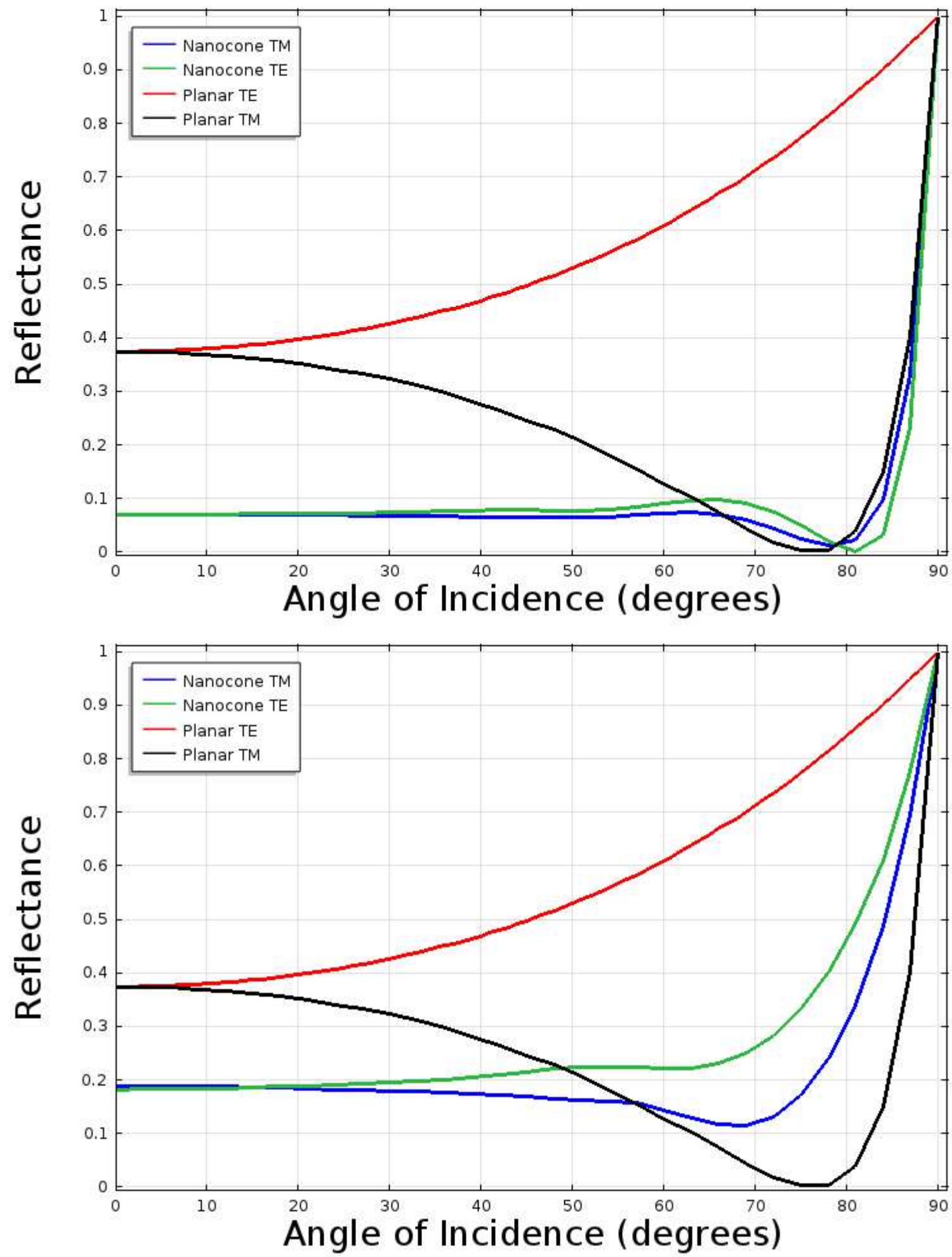

Figure 4.29. Reflectance vs. angle of incidence comparison for a $1000 \mathrm{~nm}$ height $\mathrm{x} 50 \mathrm{~nm}$ radius nanocone model and a planar interface with a $50 \mathrm{~nm} \mathrm{ZnO}$ seed layer (top) and without (bottom). 
The seed layer was then applied to the 3D quasi-randomized geometry. A $50 \mathrm{~nm}$ layer of $\mathrm{ZnO}$ was added between the bases of the cones and the substrate and the model was run again for TM and TE modes. The reflectance is plotted as a function of incident angle. This model needed 1,108,474 mesh elements and solved for 7,019,960 degrees of freedom. The geometry and mesh are shown in figure 4.30. There is a false blue coloring for contrast with the other meshed domains in order to highlight the seed layer.

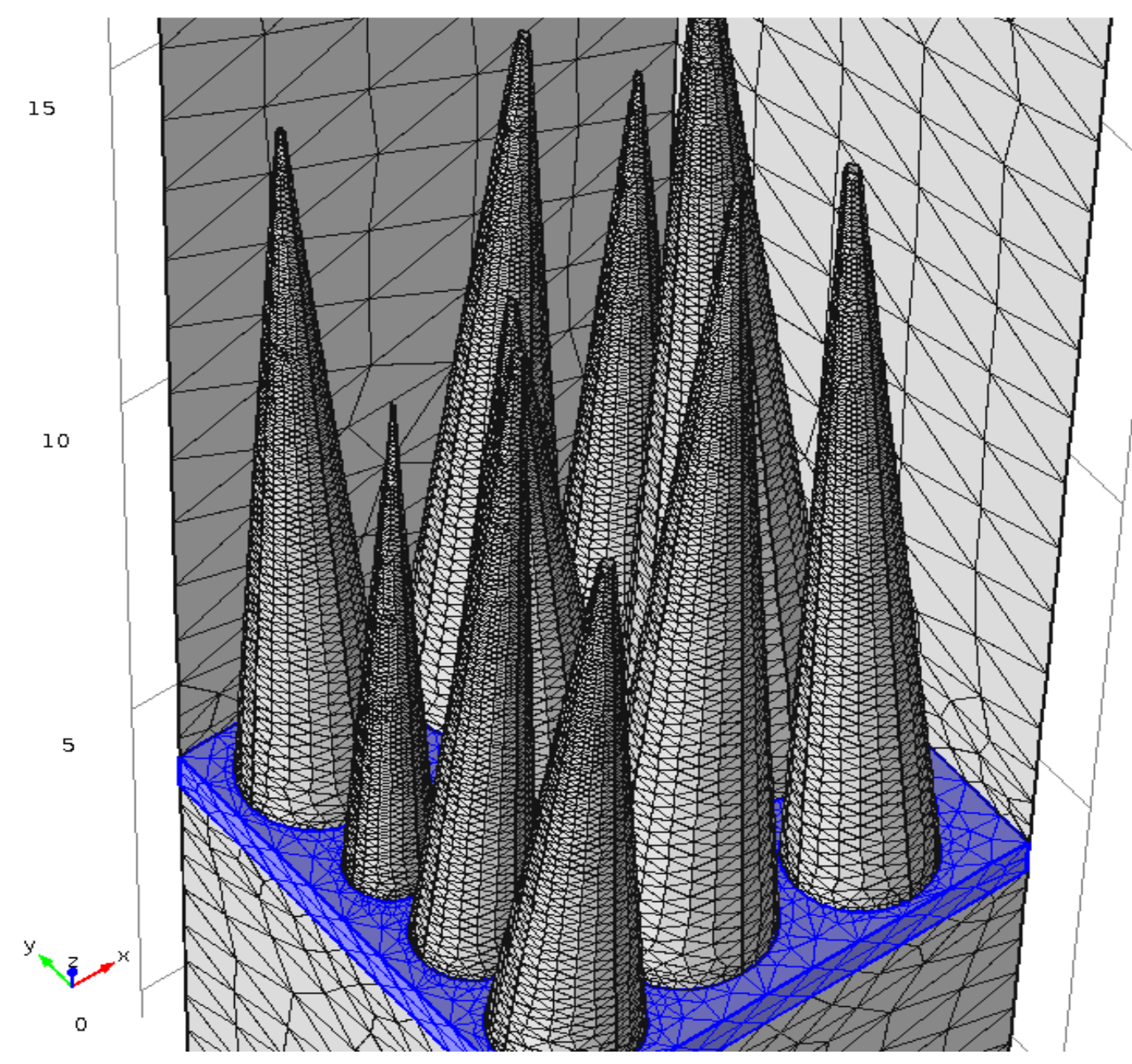

Figure 4.30. Quasi-random nanocone mesh with a $50 \mathrm{~nm}$ seed layer below the cone bases. 
The mesh needed several edge elements to be constructed. Without these, the volume elements became inverted as the mesh distorted itself to fit within the model's parameters. This involved considerable time commitment to optimize the element sizes and discrete constructions.

The reflectance simulations (figure 4.31) show a significant decrease over the model without a seed layer. This extra layer, which stems from nanostructure growth, improves the overall optical performance of the nanocones. Since the laboratory-grown structures were nanowires instead of nanocones, this is only an approximation and would need to be examined experimentally. The creation of these nanocones from nanowires would possibly require some form of etchant application.

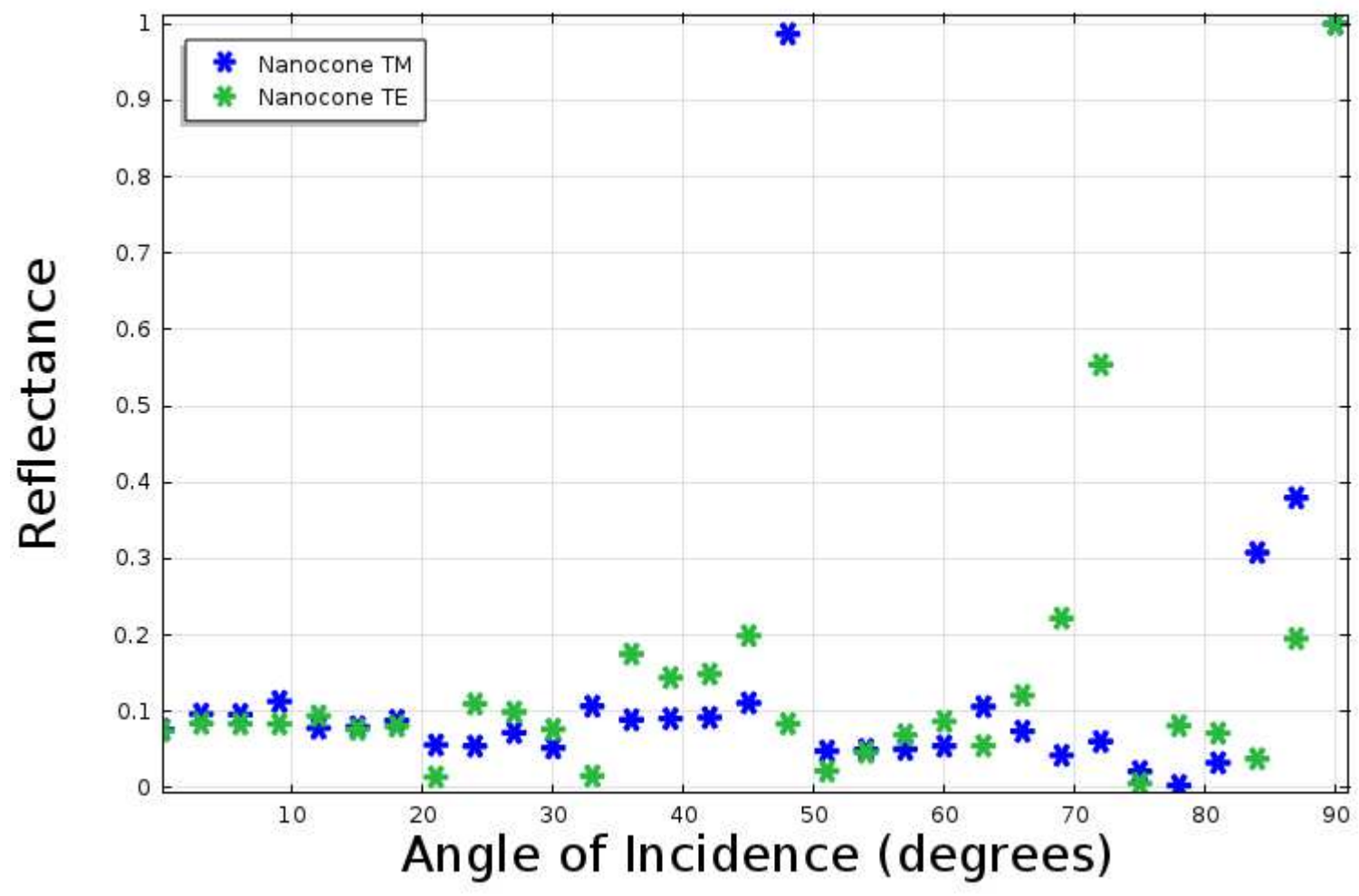

Figure 4.31. Reflectance vs. angle of incidence for quasi-random nanocones with a $50 \mathrm{~nm}$ seed layer. 
The values at $48^{\circ}$ for TM polarization and $72^{\circ}$ for TE polarization show two more possible artifacts. The reflectance is $98.697 \%$ and $55.399 \%$ respectively. Both of these values deviate greatly from the data points $3^{\circ}$ below and above them. The same methodology as before is employed to check their validity.

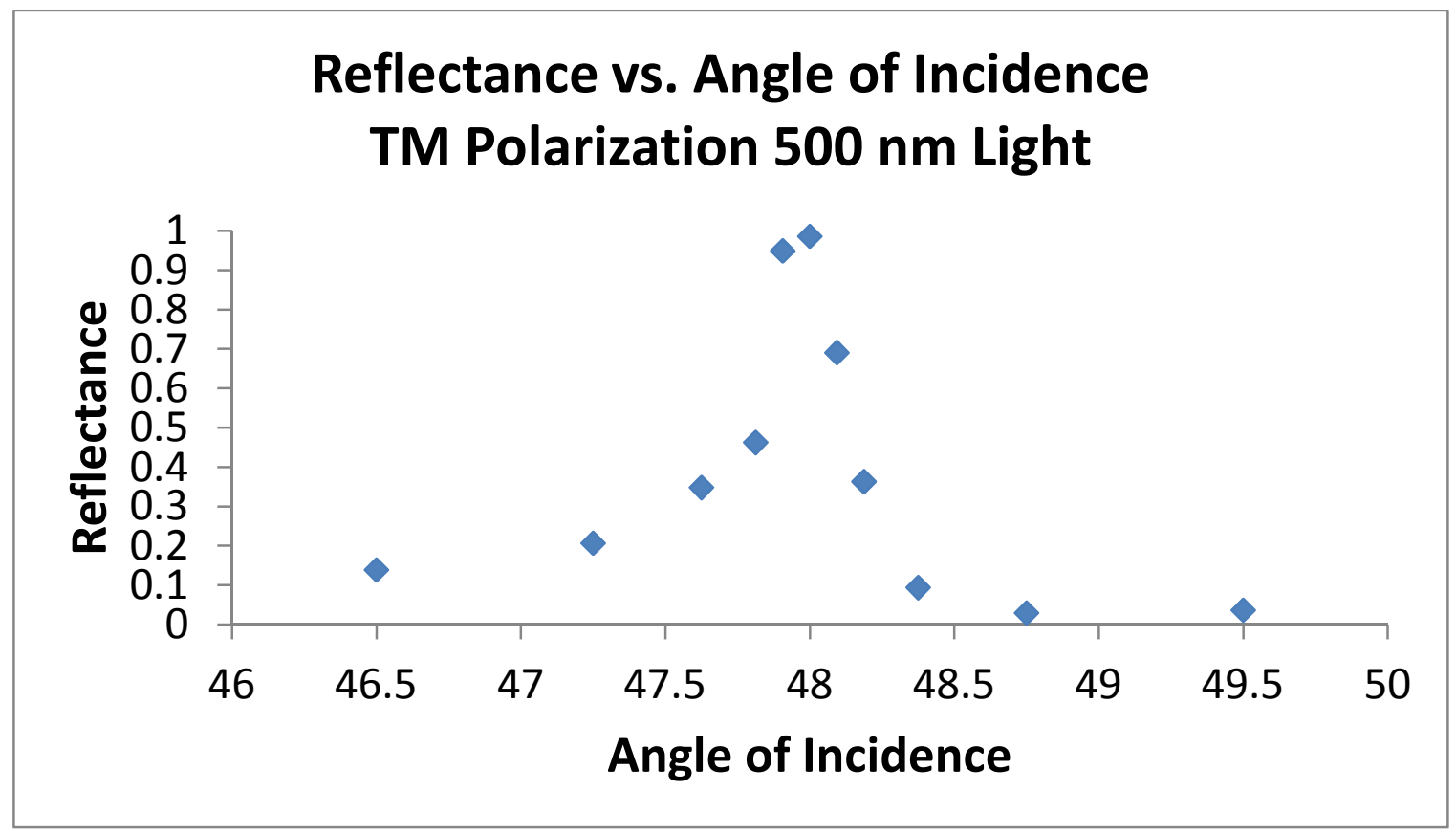

Figure 4.32. Reflectance validity check around $48^{\circ}$ for TM polarization outlying point. The model is a quasi-random nanocone with a seed layer geometry.

A possible diffraction response was also checked by varying the wavelength at the $48^{\circ}$ outlier data point in figure 4.32. The values of reflectance were $50 \%$ for $500.1 \mathrm{~nm}$ and $54 \%$ for $499.9 \mathrm{~nm}$ light. With such small deviations from $500 \mathrm{~nm}$, the magnitude of these data points should be higher if it were a resonance peak since at $48^{\circ}$ the reflectance is close to $100 \%$. A small change in wavelength should not produce such a dramatic change in the total reflection. 
The reflectance as a function of incident light angle for the $72^{\circ}$ data point from the TE plot is shown in figure 4.33. The peak is about half of the previous outliers and seems to more smoothly vary as the angle changes slightly. This is also checked for resonance using a variation of the wavelength. Adjusting the wavelength by $0.1 \mathrm{~nm}$ above and below $500 \mathrm{~nm}$ showed a large discrepancy in reflection values with $10.869 \%$ for 499.9 $\mathrm{nm}$ and $22.544 \%$ for $500.1 \mathrm{~nm}$ incident light. A real resonance peak would not drop so dramatically, nor vary so widely, with such a small change in the wavelength. This suggests the existence of an artifact at this data point.

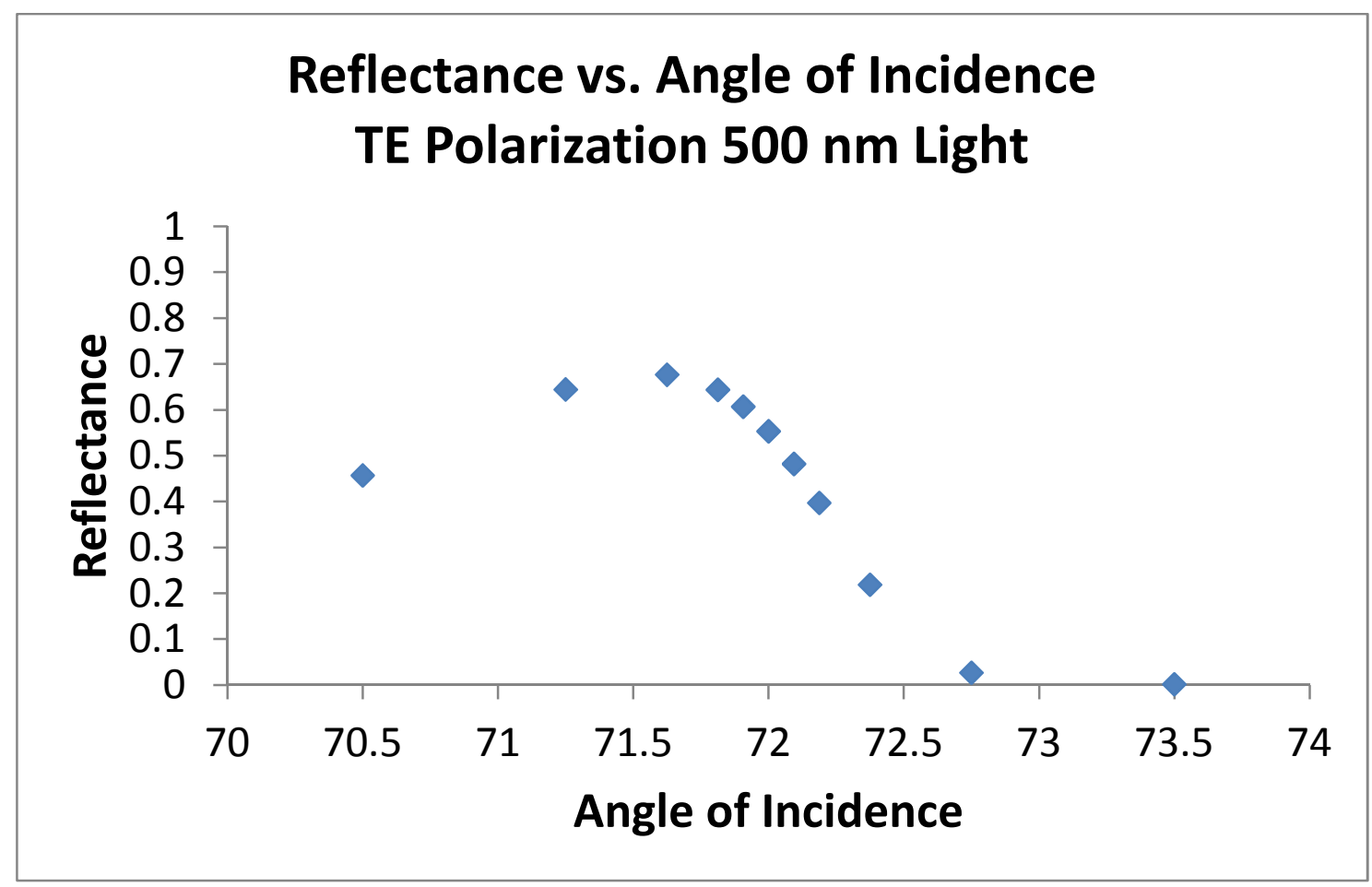

Figure 4.33. Reflectance validity check around $72^{\circ}$ for TE polarization outlying point. The model is a quasi-random nanocone with a seed layer geometry. 


\subsection{Comparison with Laboratory Results}

In the laboratory, nanowires were grown by the research group and had characteristics of high density, highly variable angles, and hexagonal structures. A scanning electron microscope image of the nanowires is displayed in figure 4.34 and is contrasted with the quasi-random nanocone geometry from the computational models (figure 4.35). The nanowire sample image is $6.2 \mu \mathrm{m}^{2}$ and the nanocone model is $375 \mathrm{~nm}^{2}$. This visually distorts the density comparison between the images.

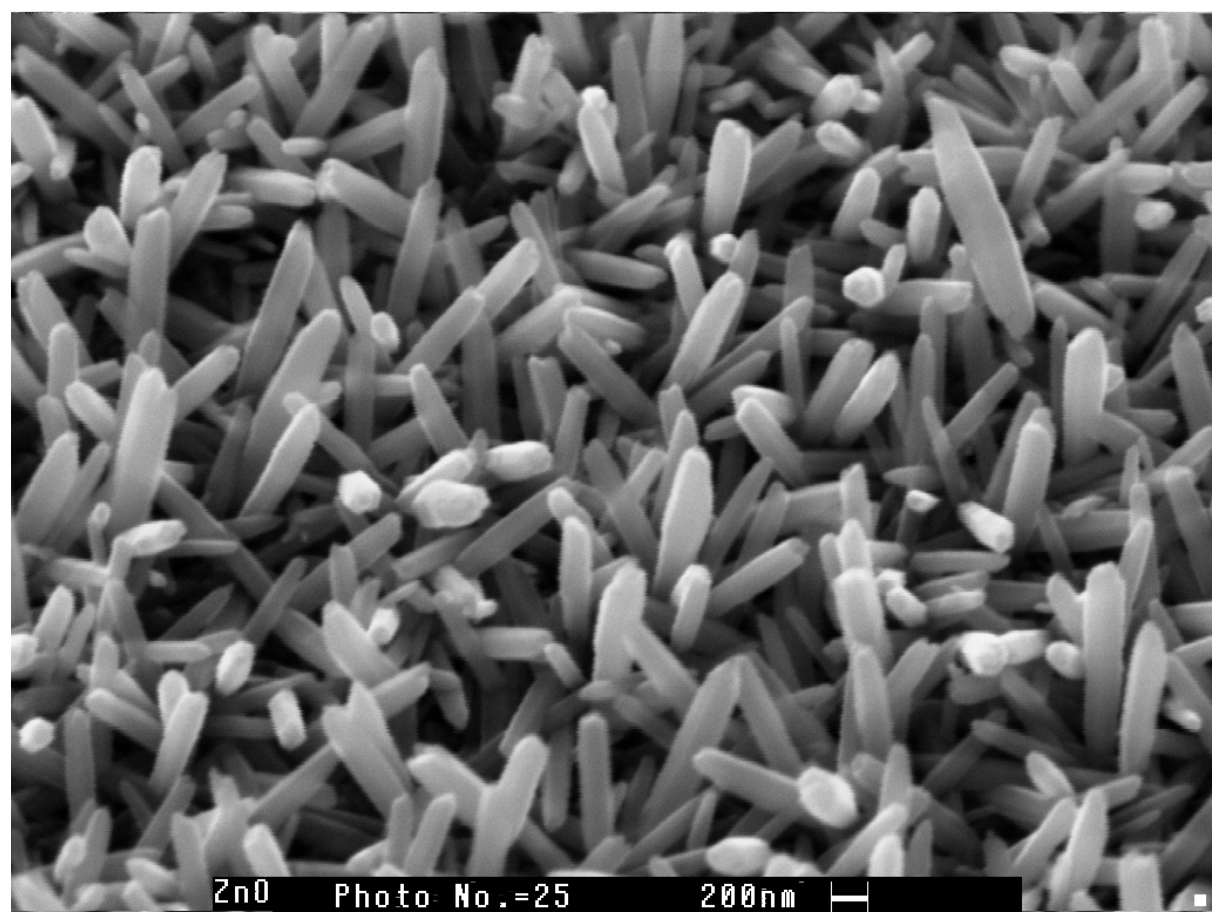

Figure 4.34. Scanning electron microscope image of $\mathrm{ZnO}$ nanowires on Si substrate. 


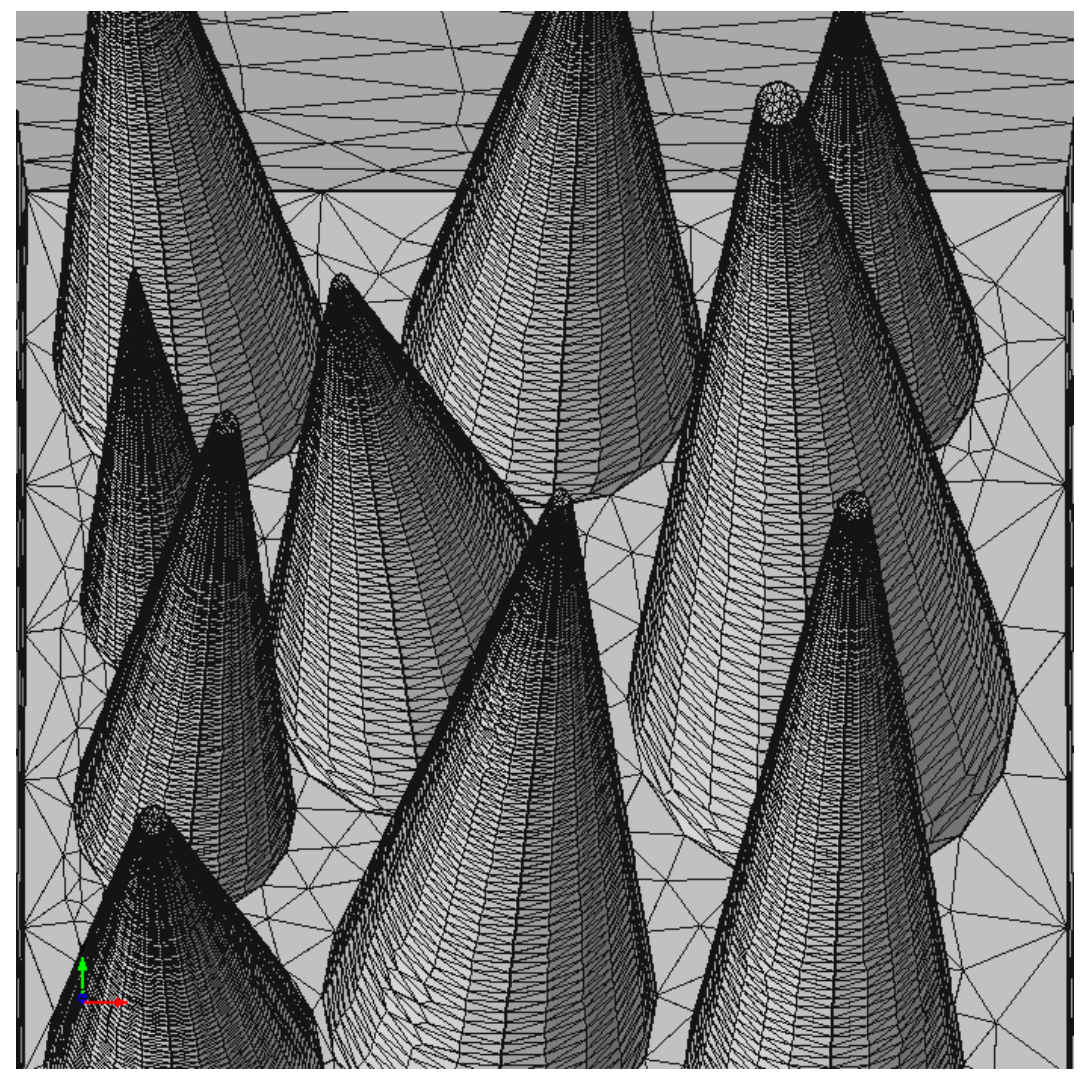

Figure 4.35. Quasi-random nanocone computational model.

A density comparison reveals a slight variation between the sample and model. A 1500 $\mathrm{nm}^{2}$ selection from the nanowire sample was chosen for a density comparison (figure 4.36). The larger area increased the ability to visually count the wires and increases the statistical variation of wire placement. The total wire count was 48 . Dividing this by four, to match the nanocone model size, gives a count of 12 nanowires per $375 \mathrm{~nm}^{2}$. This is slightly hirgher than the 10 nanocones per $375 \mathrm{~nm}^{2}$ from the model. 


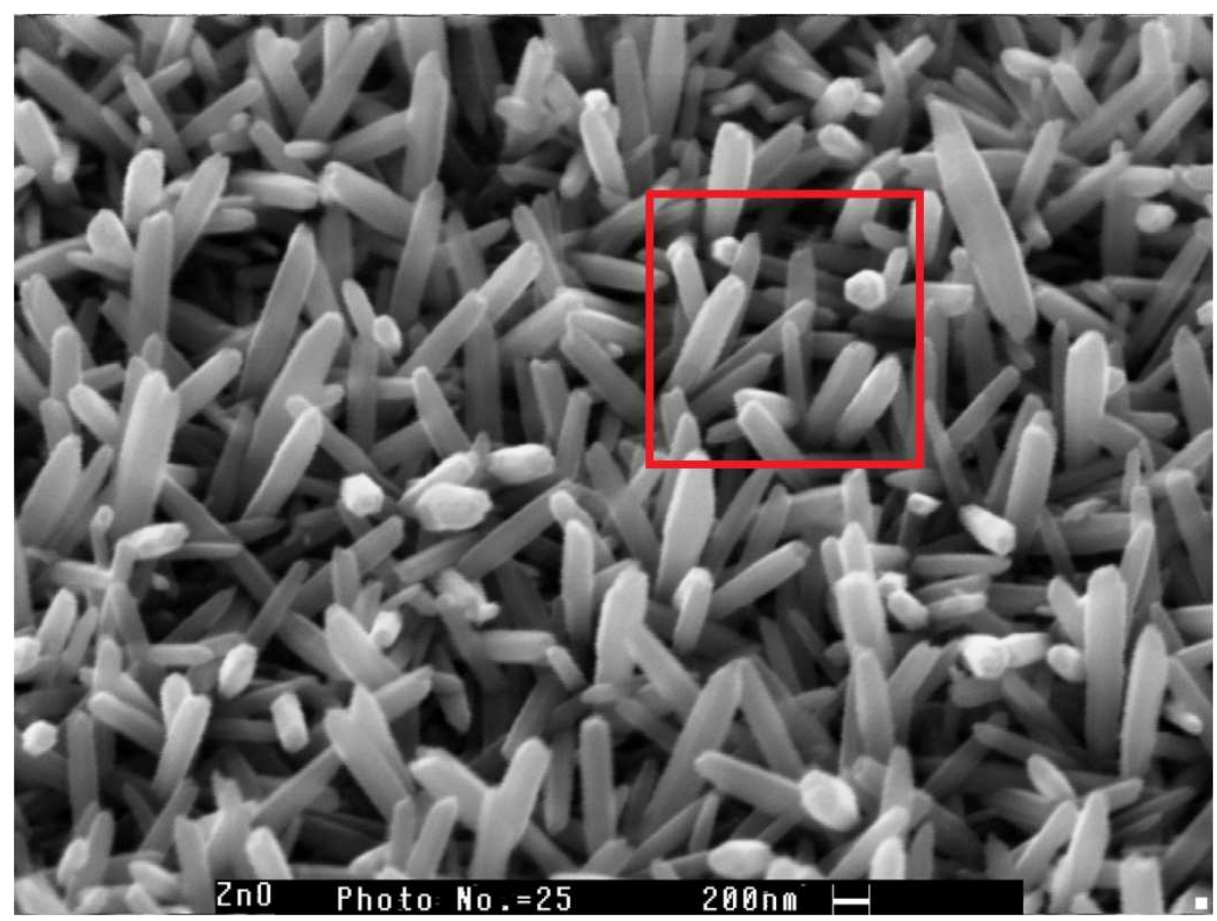

Figure 4.36. SEM image of $\mathrm{ZnO}$ nanowires on Si substrate. A $1500 \mathrm{~nm}^{2}$ area (red) is used to calculate the nanowire density.

Reflection intensity plots of the nanowire samples in figure 4.37 range from $-90^{\circ}$ to $90^{\circ}$ degrees of the angle $\theta$. In this experiment the reflection is measured with a small area detector at the specular angle. In this arrangement the scattering angle is $2 \theta$, and the plot shows reflected intensity vs. scattering angle, $2 \theta$. This experimental arrangement is hence not the same as assumed in this thesis work. An important aspect of the experimental results is the disappearance of polarization dependence due to the randomized structures. This is reproduced in the quasi-random, periodic boundary model. The scattering of light from the geometry is no longer orderly and does not preserve the TM and TE characteristics. 


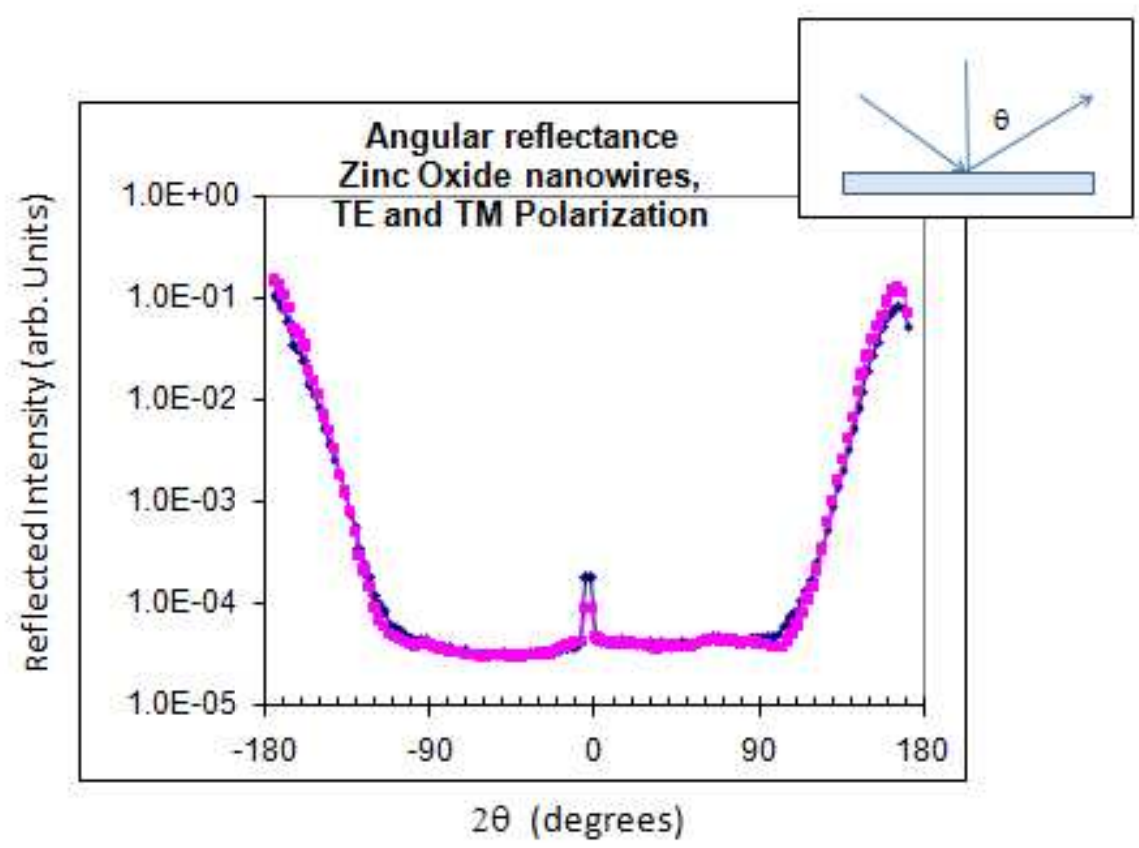

Figure 4.37. Experimental reflectance from randomly oriented $\mathrm{ZnO}$ nanowires.

\subsection{Wavelength Dependence of Quasi-Random Nanocone Model with Seed Layer}

The quasi-random model with a seed layer was checked for reflectance response due to varying wavelength. The wavelength range used was $450 \mathrm{~nm}$ to $750 \mathrm{~nm}$. The incident wavelength was swept for both TM and TE polarizations and shows a reflectance value of $\sim 7 \%$, on average. Figure 4.38 shows both the TM and TE mode plots for light with perpendicular incidence. As expected, the polarization dependence disappears from the randomized geometry causing heavy scattering. This causes the data points to overlap appreciably. 


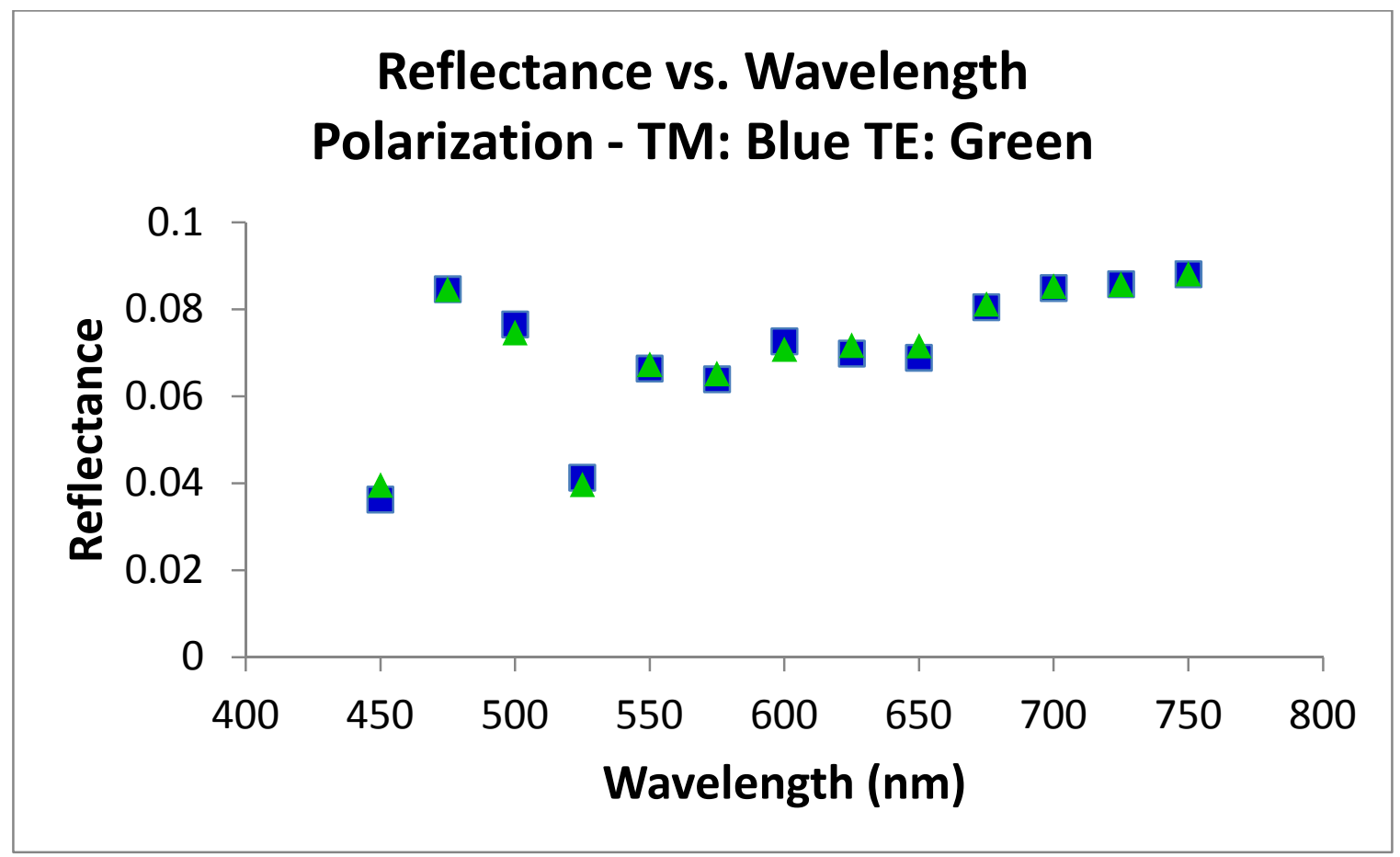

Figure 4.38. Wavelength dependence of reflectance for TM and TE polarization incident at perpendicular incidence on a quasi-randomized nanocone geometry.

\subsection{Discussion and Future Work}

The randomization of the structures in the models increases the required computational power. These models need extra refinement at geometrically complex areas. This refinement slows runtime and even overstretches the memory of the server causing frequent crashes. There is a trade-off between performance and accuracy in the models which is seen in jittery curves and in spikes of reflectance values near singular points going up to $100 \%$. The reflectance at these angles was examined and shown to be likely caused by artifacts due to the lack of mesh refinement. Decreased mesh elements sizes increased the number of mesh elements, increasing the degrees of freedom and equations for which to solve, thereby increasing the runtime. The RAM utilized for the solutions 
averaged $\sim 152 \mathrm{~GB}$ of a total $176 \mathrm{~GB}$. This was after a server computer was built to handle such computations. Previously, the models simply ran out of memory and could not be completed.

The computational demand on the system required the limitation of models to the optical properties. While only optical phenomena were considered, the challenges were many. The models constructed were plagued by inaccurate results for well over a year resulting in inaccurate solutions for even the simplest cases. The early models showed wavy patterns in the fields which were artifacts caused by an insufficient mesh refinement. An increase in RAM eliminated this problem. Problems stemmed from the reflectance measurements showing significant flux in values, greater than $100 \%$ of incident light, and even negative values in the early stages of development. These were overcome by reformulation of the problem into S-matrix calculations and a two port system, increased memory and computational power for the hardware used, and persistence from the modeler.

As for the physics, the nanocone model showed a good overall reduction of reflectance when compared with planar interfaces. An overall decrease in the reflectance values were achieved. The reflectance data did not exactly paralleled the laboratory experiments, but differing geometric shapes restricted the comparison. The computations did perform well enough to validate the model's accuracy. Yet, the results are not yet refined enough to allow for an accurate description of a working device. In solar cells, it is unlikely the structures will be outside of a protective layer. This adds a minimum of one more medium through which light must travel before coming into contact with the 
nanostructures. This outer layer will typically be an acrylic or glass and will not significantly reduce the transmission, but it will not be ideal. However, an inversion of the nanocones on the backside of the protective transparent cover may have similar optical enhancements when a heterojunction is created with a p-type medium. This remains another option for a real device and a simulated model would be beneficial to enhance the design.

The inclusion of the seed layer, as often used in deposition techniques, allowed the reflectance improvement to be more competitive with the quintic profiles calculated in the 1980's from effective medium models. Our calculations showed that a $50 \mathrm{~nm}$ seed layer lowers the reflectance at small angles to approximately $8 \%$. This is less than half the reflectance typically found in nanocone surfaces without seed layers, which was calculated to be $\sim 18 \%$. We were also able to confirm experimental findings that the polarization dependence of the reflectance gets lost on random structured surfaces.

Overall, we can therefore state that exact solutions to Maxwell's equations for simple optical problems is now becoming feasible with desktop computers enhanced to $\sim 180 \mathrm{~GB}$ RAM, and with commercially available programs.

Of course, our results do not yet accurately represent the entirety of a working solar cell as we disregarded completely the physics of electron transport, the inclusion of a p-type material to create the junction, the outer contacts which block absorption, and thermal heating to name a few of the important properties to model. The desire to model complete cells in the future remains an intriguing option for further work. 


\section{References}

[1] S. E. I. Association, "Solar market insight report," tech. rep., 2012 Q2.

[2] U. E. I. Administration, “Annual energy outlook 2013 early release overview,” tech. rep., 2013.

[3] P. Würfel and U. Würfel, Physics of Solar Cells: From Basic Principles to Advanced Concepts. Wiley. com, 2009.

[4] K. Sattler, "Handbook of nanophysics: Nanoelectronics and nanophotonics. chapter 31: Biomimetics: Photonic nanostructures," KD Sattler, Taylor and Francis group, vol. 6, pp. 31-1, 2010.

[5] Jan-Henning Dirks, "Technical Applications of Biomimetic Nanostructures."

[6] M. Zeman, Introduction to Photovoltaic Solar Energy, ch. 4, pp. 4.1-4.2. Delft University of Technology, 2003.

[7] D. R. Evans, Microelectronic Device Fabrication I, p. 165. Portland State University, 2012.

[8] W. Shockley and H. J. Queisser, "Detailed Balance Limit of Efficiency of p-n Junction Solar Cells," Journal of Applied Physics, vol. 32, no. 3, pp. 510-519, 1961.

[9] T. Tiedje, E. Yablonovitch, G. D. Cody, and B. G. Brooks, "Limiting Efficiency of Silicon Solar Cells," Electron Devices, IEEE Transactions on, vol. 31, no. 5, pp. 711$716,1984$. 
[10] M. Zeman, Introduction to Photovoltaic Solar Energy, vol. 1, ch. 5, pp. 5.1-5.8. Delft University of Technology, 2003.

[11] PVEducation.org, "Optical Losses."

[12] S. Chattopadhyay, Y. Huang, Y. Jen, A. Ganguly, K. Chen, and L. Chen, "Antireflecting and photonic nanostructures," Materials Science and Engineering: R: Reports, vol. 69, no. 1, pp. 1-35, 2010.

[13] M. Born and E. Wolf, Principles of optics: electromagnetic theory of propagation, interference and diffraction of light. Cambridge university press, seventh (expanded) ed., 1999.

[14] PVEducation.org, "Anti-Reflection Coatings."

[15] Y.-J. Lee, D. S. Ruby, D. W. Peters, B. B. McKenzie, and J. W. Hsu, “Zno nanostructures as efficient antireflection layers in solar cells," Nano letters, vol. 8, no. 5, pp. 1501-1505, 2008.

[16] PVEducation.org, "Light Trapping."

[17] E. D. Palik, Handbook of Optical Constants of Solids: Index, vol. 3. Academic press, 1998.

[18] J. Bae, J.-I. Hong, W. H. Han, Y. J. Choi, and R. L. Snyder, "Superior field emission properties of zno nanocones synthesized by pulsed laser deposition," Chemical physics letters, vol. 475, no. 4, pp. 260-263, 2009. 
[19] M. Coakley, "Growth and optical characterization of zinc oxide nanowires for anti-reflection coatings for solar cells," Master's thesis, Portland State University, 2011.

[20] Oak Ridge National Laboratory, "Metamaterials."

[21] F. L. Pedrotti, Introduction To Optics, 3/E. Pearson Education India, 2008.

[22] T. C. Choy, Effective medium theory: principles and applications, vol. 102. Oxford University Press, 1999.

[23] P. P. Altermatt, Y. Yang, T. Langer, A. Schenk, and R. Brendel, "Simulation of optical properties of si wire cells," in Photovoltaic Specialists Conference (PVSC), 2009 34th IEEE, pp. 000972-000977, IEEE, 2009.

[24] K. Sattler, "Handbook of nanophysics: Nanotube and nanowires. chapter 25: Optical properties of anisotropic metamaterial nanowires," $L L C, U S A$, vol. 4, pp. 25-1, 2011.

[25] "Zinc Oxide Nanowires on Silicon Substrate."

[26] R. K. Wangsness, Electromagnetic fields, vol. 1. John Wiley \& Sons, Inc., 1986.

[27] Leung, P, “Electromagnetic Fields and Interactions: Course Notes," 2009-2010.

[28] Wikimedia Commons, "Electromagnetic wave transmission: TE, B-field," September 2012.

[29] D. J. Griffiths and R. College, Introduction to electrodynamics, vol. 3. prentice Hall Upper Saddle River, NJ, 1999. 
[30] Wikimedia Commons, "Electromagnetic wave transmission: TE, H-field," September 2012 .

[31] Wolski, A, "Physics 370 - Advanced Electromagnetism, Part 4: Waves on Boundaries: Class notes.”

[32] Wikimedia Commons, "Electromagnetic wave transmission: TM average," September 2012.

[33] A. J. Davies, The finite element method. Clarendon Press Oxford, 1980.

[34] O. de Weck and I. Y. Kim, "Lecture Notes: Engineering Design and Rapid Prototyping, Finite Element Method," 2004.

[35] O. Schenk and K. Gartner, PARDISO User Guide Version 4.1.2. PARDISO Solver Project, 2011.

[36] A. Rennings, "Element Method \& COMSOL Multiphysics, Part II: Electrodynamic Fields,” 2009.

[37] W. H. Press, Numerical recipes in Fortran 77: the art of scientific computing, vol. 1. Cambridge university press, 1992.

[38] O. Schenk and K. Gärtner, "Solving unsymmetric sparse systems of linear equations with pardiso," in Computational Science-ICCS 2002, pp. 355-363, Springer, 2002.

[39] C. Multiphysics, "Rf module user's guide," 2012. 
[40] P.-T. Leung, "Lecture notes: Electromagnetic fields and interactions," 2009-2010.

[41] W. H. Southwell, "Gradient-index antireflection coatings," Optics letters, vol. 8, no. 11, pp. 584-586, 1983. 